\title{
Total Syntheses of Natural Tubelactomicins B, D, and E: Establishment of Their Stereochemistries
}

\author{
Kiyoto Sawamura, Keigo Yoshida, Akari Suzuki, Toru Motozaki, Ikuko Kozawa, Takashi Hayamizu, \\ Ryosuke Munakata, Ken-ichi Takao, and Kin-ichi Tadano*
}

Department of Applied Chemistry, Keio University, Hiyoshi, Kohoku-ku, Yokohama 223-8522, Japan

\section{Supporting Information}

\section{Contents}

$\begin{array}{lc}\text { Experimental Section } & \text { S1-S43 } \\ { }^{1} \mathrm{H} \text { and }{ }^{13} \mathrm{C} \text { NMR Spectra of New Compounds } & \text { S44-S143 }\end{array}$

\section{Experimental Section}

Specific rotations were measured in a $10 \mathrm{~mm}$ cell. ${ }^{1} \mathrm{H}$ NMR spectra were recorded at $270 \mathrm{MHz}$ or at $300 \mathrm{MHz}$ in $\mathrm{CDCl}_{3}$ with tetramethylsilane as an internal standard unless otherwise described. ${ }^{13} \mathrm{C}$ NMR spectra were recorded at $68 \mathrm{MHz}$ or at $75 \mathrm{MHz}$ in $\mathrm{CDCl}_{3} \cdot{ }^{1} \mathrm{H}$ NMR spectral data are tabulated in the order; multiplicity (s, singlet; d, doublet; t, triplet; q, quartet; m, multiplet), coupling constant, number of protons. High-resolution mass spectra (HRMS) were measured by the EI method (70 eV) unless otherwise noted. Thin-layer chromatography (TLC) was performed with a glass plate coated with Kieselgel 60 F254 (Merck). The crude reaction mixtures and extractive materials were purified by 
chromatography on silica gel Daisogel IR-60 (Daiso Co., Ltd.) or Wakogel C-300 (Wako Pure Chemical Industries). Unless otherwise described, reactions were carried out at ambient temperature. Combined organic extracts were dried over anhydrous $\mathrm{Na}_{2} \mathrm{SO}_{4}$. Solvents were removed from reaction mixture or combined organic extracts by concentration under reduced pressure using an evaporator with a water bath at $30-40{ }^{\circ} \mathrm{C}$.

\section{Ethyl (2E,7R)-7-(tert-butyldiphenylsilyloxy)-2-methyloct-2-enoate (14).}

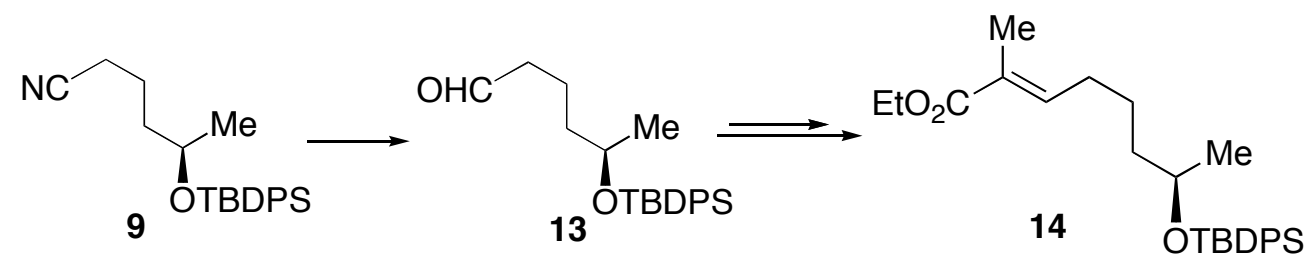

The following reaction was carried out under argon. To a cooled $\left(-78^{\circ} \mathrm{C}\right)$, stirred solution of $9^{1 \mathrm{a}}$ $(1.77 \mathrm{~g}, 5.04 \mathrm{mmol})$ in toluene $(30 \mathrm{~mL})$ was added DIBAL-H $(7.5 \mathrm{~mL}$ of $1.01 \mathrm{M}$ solution in toluene, 7.6 mmol). The mixture was stirred at $-78{ }^{\circ} \mathrm{C}$ for $1.5 \mathrm{~h}$ and then quenched with saturated $\mathrm{H}_{2} \mathrm{O}(5 \mathrm{~mL})$. After being warmed to room temperature, this was diluted with aqueous solution $(100 \mathrm{~mL})$ of potassium sodium (+)-tartrate tetrahydrate $(1.55 \mathrm{~g})$ and added $\mathrm{Et}_{2} \mathrm{O}(5 \mathrm{~mL})$. The mixture was warmed to room temperature and stirred vigorously for $2 \mathrm{~h}$. This was extracted with $\mathrm{Et}_{2} \mathrm{O}(50 \mathrm{~mL} \times 3)$. The combined organic layers were dried and concentrated in vacuo to give a crude aldehyde $\mathbf{1 3}$, which was used in the next step without further purification.

The following reaction was carried out under argon. To a stirred solution of the crude $\mathbf{1 3}$ obtained above in benzene $(30 \mathrm{~mL})$ was added $\mathrm{Ph}_{3} \mathrm{P}=\mathrm{C}(\mathrm{Me}) \mathrm{CO}_{2} \mathrm{Et}(2.74 \mathrm{~g}, 7.56 \mathrm{mmol})$. The mixture was stirred for $23.5 \mathrm{~h}$ and $\mathrm{Ph}_{3} \mathrm{P}=\mathrm{C}(\mathrm{Me}) \mathrm{CO}_{2} \mathrm{Et}(916 \mathrm{mg}, 2.53 \mathrm{mmol})$ was added. The mixture was stirred for $6 \mathrm{~h}$ and then concentrated in vacuo. The residue was purified by column chromatography on silica gel (EtOAc/hexane, 1:50) to give $2.01 \mathrm{~g}(91 \%)$ of $\mathbf{1 4}$ as a colorless oil; TLC, $\mathrm{R}_{f} 0.77$ (EtOAc/hexane, 1:4); $[\alpha]_{\mathrm{D}}^{21}+22.2\left(c 2.03, \mathrm{CHCl}_{3}\right) ;{ }^{1} \mathrm{H}$ NMR $(300 \mathrm{MHz}) \delta 1.05(\mathrm{~s}, 9 \mathrm{H}), 1.06(\mathrm{~d}, J=6.2 \mathrm{~Hz}, 3 \mathrm{H}), 1.29(\mathrm{t}, J=$ $7.1 \mathrm{~Hz}, 3 \mathrm{H}), 1.36-1.54(\mathrm{~m}, 4 \mathrm{H}), 1.77(\mathrm{~d}, J=1.3 \mathrm{~Hz}, 3 \mathrm{H}), 2.05(\mathrm{dt}, J=6.6,7.5 \mathrm{~Hz}, 2 \mathrm{H}), 3.84(\mathrm{tq}, J=5.6$ 
$6.2 \mathrm{~Hz}, 1 \mathrm{H}), 4.19(\mathrm{q}, J=7.1 \mathrm{~Hz}, 2 \mathrm{H}), 6.69(\mathrm{dt}, J=1.3,7.5 \mathrm{~Hz}, 1 \mathrm{H}), 7.32-7.44,7.63-7.73$ (m, total 10H);

${ }^{13} \mathrm{C}$ NMR $(68 \mathrm{MHz}) \delta 12.3,14.3,19.2,23.2,24.1,27.0 \times 3,28.6,39.0,60.4,69.2,127.3 \times 2,127.5 \times 2$,

$127.8,129.4,129.5,134.5,134.8,135.9 \times 4,142.1,168.3$; IR (neat) 3080, 2930, 2860, 1720, 1650, 1430

$\mathrm{cm}^{-1}$; HRMS calcd for $\mathrm{C}_{26} \mathrm{H}_{35} \mathrm{O}_{3} \mathrm{Si}\left(\mathrm{M}^{+}-\mathrm{CH}_{3}\right) m / z$ 423.2356, found 423.2355.

\section{(2E,7R)-7-(tert-Butyldiphenylsilyloxy)-2-methyloct-2-enol (15).}

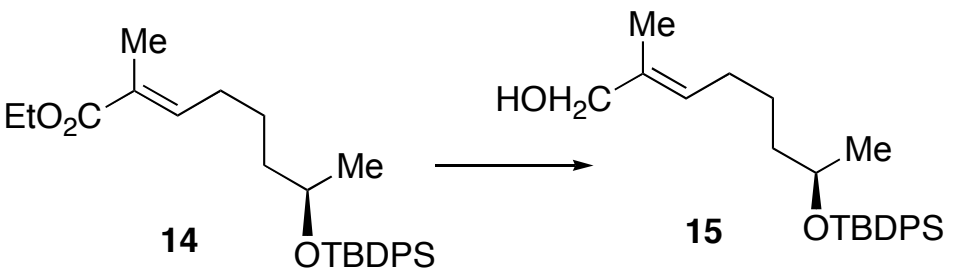

The following reaction was carried out under argon. To a cooled $\left(-78^{\circ} \mathrm{C}\right)$, stirred solution of 14 (705 mg, $1.61 \mathrm{mmol})$ in $\mathrm{CH}_{2} \mathrm{Cl}_{2}(10 \mathrm{~mL})$ was added DIBAL-H (4.0 mL, 1.0 M solution in toluene, 4.0 mmol). The mixture was stirred at $-78{ }^{\circ} \mathrm{C}$ for $1 \mathrm{~h}$ and quenched with $\mathrm{H}_{2} \mathrm{O}$. This was diluted with $\mathrm{CH}_{2} \mathrm{Cl}_{2}(10 \mathrm{~mL})$, and an aqueous solution $(20 \mathrm{~mL})$ of potassium sodium $(+)$-tartrate tetrahydrate $(2.85 \mathrm{~g})$ was added. The mixture was stirred vigorously for $2 \mathrm{~h}$ and the organic layer was separated. The aqueous layer was extracted with $\mathrm{CH}_{2} \mathrm{Cl}_{2}(10 \mathrm{~mL} \times 3)$. The combined organic layers were dried and concentrated in vacuo. The residue was purified by chromatography on silica gel (EtOAc/hexane, 1:10) to give $633 \mathrm{mg}(99 \%)$ of $\mathbf{1 5}$ as a colorless oil: TLC, $\mathrm{R}_{f} 0.38\left(\right.$ EtOAc/hexane, 1:4); $[\alpha]^{21}{ }_{\mathrm{D}}+21.1(c$ 2.29, $\left.\mathrm{CHCl}_{3}\right) ;{ }^{1} \mathrm{H}$ NMR $(300 \mathrm{MHz}) \delta 1.05(\mathrm{~s}, 9 \mathrm{H}), 1.06(\mathrm{~d}, J=5.8 \mathrm{~Hz}, 3 \mathrm{H}), 1.24-1.56(\mathrm{~m}, 4 \mathrm{H}), 1.61(\mathrm{~s}, 3 \mathrm{H})$, $1.92(\mathrm{dt}, J=7.3,6.8 \mathrm{~Hz}, 2 \mathrm{H}), 3.84(\mathrm{tq}, J=6.0,5.8 \mathrm{~Hz}, 1 \mathrm{H}), 5.32(\mathrm{t}, J=7.3 \mathrm{~Hz}, 1 \mathrm{H}), 7.30-7.46,7.62-7.74$ $(\mathrm{m}$, total $10 \mathrm{H}) ;{ }^{13} \mathrm{C} \mathrm{NMR}(68 \mathrm{MHz}) \delta 13.6,19.2,23.2,25.0,27.0 \times 3,27.5,39.0,69.0,69.4,126.4$, $127.4 \times 2,127.4 \times 2,129.4,129.4,134.6,134.7,134.8,135.9 \times 4$; IR (neat) $3300,3080,2930,2860$, $1430 \mathrm{~cm}^{-1}$; HRMS calcd for $\mathrm{C}_{24} \mathrm{H}_{33} \mathrm{O}_{2} \mathrm{Si}\left(\mathrm{M}^{+}-\mathrm{CH}_{3}\right) \mathrm{m} / z$ 381.2250, found 381.2251. 
(2E,7R)-7-(tert-Butyldiphenylsilyloxy)-2-methyloct-2-enal (16).

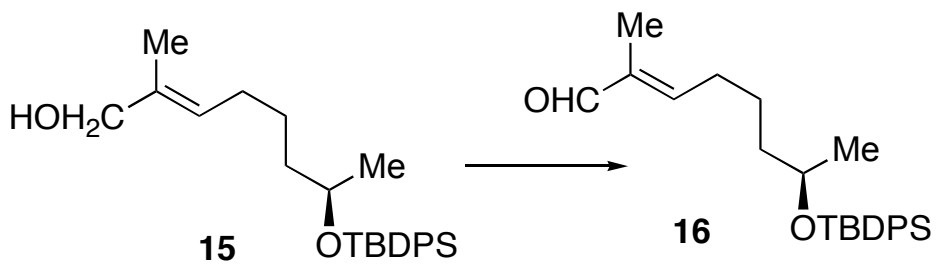

To a cooled $\left(0^{\circ} \mathrm{C}\right)$, stirred solution of $15(1.33 \mathrm{~g}, 7.56 \mathrm{mmol})$ in $\mathrm{CH}_{2} \mathrm{Cl}_{2}(26 \mathrm{~mL})$ was added $\mathrm{MnO}_{2}$ (13.3 g, $153 \mathrm{mmol})$. The mixture was stirred for $15 \mathrm{~min}$ and the insoluble materials were filtered off and washed well with EtOAc. The combined filtrate and washings were concentrated in vacuo. The residue was purified by column chromatography on silica gel (EtOAc/hexane, 1:25) to give $1.17 \mathrm{~g}$ (89\%) of 16 as a colorless oil; TLC $\mathrm{R}_{f} 0.77$ (EtOAc/hexane, 1:4) $[\alpha]^{28}{ }_{\mathrm{D}}+33.0\left(c 2.30, \mathrm{CHCl}_{3}\right) ;{ }^{1} \mathrm{H}$ NMR $(300 \mathrm{MHz}) \delta 1.05(\mathrm{~s}, 9 \mathrm{H}), 1.09(\mathrm{~d}, J=6.2 \mathrm{~Hz}, 3 \mathrm{H}), 1.34-1.55(\mathrm{~m}, 4 \mathrm{H}), 1.68(\mathrm{~d}, J=0.6 \mathrm{~Hz}, 3 \mathrm{H}), 2.22$ (dt, $J=7.3,6.8 \mathrm{~Hz}, 2 \mathrm{H}), 3.87(\mathrm{~m}, 1 \mathrm{H}), 6.37(\mathrm{dt}, J=0.6,7.3 \mathrm{~Hz}, 1 \mathrm{H}), 7.32-7.46,7.62-7.72(\mathrm{~m}$, total $10 \mathrm{H}) ;{ }^{13} \mathrm{C}$ NMR $(68 \mathrm{MHz}) \delta 9.1,19.2,23.2,23.9,27.0 \times 3,28.8,38.8,69.1,127.4 \times 2,127.5 \times 2,129.5,129.5$, 134.4, 134.6, $135.8 \times 4,139.4,154.8,195.3$; IR (neat) 3070, 3050, 2970, 2930, 2890, 2860, 2710, 1700, 1680, 1590, $1470 \mathrm{~cm}^{-1}$; HRMS calcd for $\mathrm{C}_{21} \mathrm{H}_{25} \mathrm{O}_{2} \mathrm{Si}\left(\mathrm{M}^{+}-t\right.$-Bu) $m / z$ 337.1624, found 337.1624.

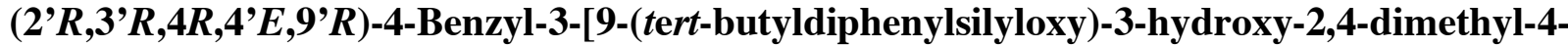
decen-1-oyl]-2-oxazolidinone (17).

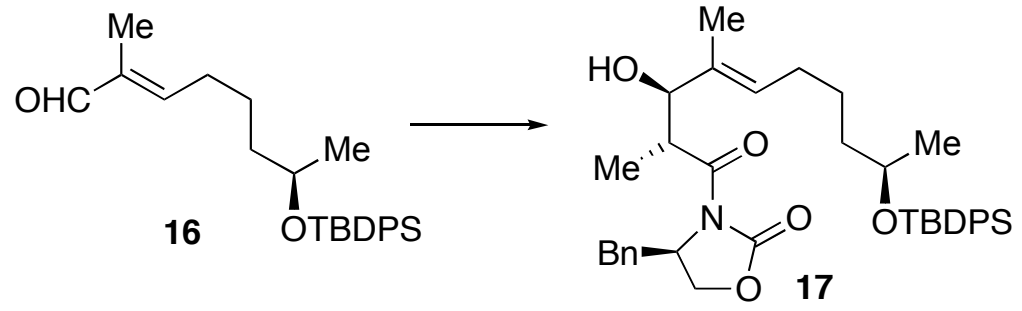

The following reaction was carried out under argon. To a cooled $\left(0^{\circ} \mathrm{C}\right)$, stirred solution of $(4 R)-4$ benzyl-3-propionyl-2-oxazolidinone $(560 \mathrm{mg}, 2.40 \mathrm{mmol})$ in $\mathrm{CH}_{2} \mathrm{Cl}_{2}(10 \mathrm{~mL})$ was added $\mathrm{Bu}_{2} \mathrm{BOTf}$ (2.8 $\mathrm{mL}$ of $1.0 \mathrm{M}$ solution in $\left.\mathrm{CH}_{2} \mathrm{Cl}_{2}, 2.8 \mathrm{mmol}\right)$. The mixture was stirred at $0{ }^{\circ} \mathrm{C}$ for 20 min and $\mathrm{Et}_{3} \mathrm{~N}(0.47$ $\mathrm{mL}, 3.7 \mathrm{mmol}$ ) was added. The mixture was stirred at $0{ }^{\circ} \mathrm{C}$ for $1 \mathrm{~h}$ and cooled to $-78{ }^{\circ} \mathrm{C}$. To this was 
added a solution of $\mathbf{1 6}(527 \mathrm{mg}, 1.60 \mathrm{mmol})$ in $\mathrm{CH}_{2} \mathrm{Cl}_{2}(4 \mathrm{~mL})$ at $-78{ }^{\circ} \mathrm{C}$. The mixture was allowed to warm to $0{ }^{\circ} \mathrm{C}$ for $4 \mathrm{~h}$ and quenched with saturated aqueous $\mathrm{NH}_{4} \mathrm{Cl}$. The mixture was stirred at $0{ }^{\circ} \mathrm{C}$ for $10 \mathrm{~min}$ and $\mathrm{MeOH}(4 \mathrm{~mL})$ and $\mathrm{H}_{2} \mathrm{O}_{2}(2 \mathrm{~mL})$ were added. The mixture was stirred at $0{ }^{\circ} \mathrm{C}$ for $1 \mathrm{~h}$ and diluted with saturated aqueous $\mathrm{NH}_{4} \mathrm{Cl}(40 \mathrm{~mL})$. The aqueous layer was extracted with $\mathrm{CH}_{2} \mathrm{Cl}_{2}(20 \mathrm{~mL} \times$ 3). The combined organic layers were washed with $10 \%$ aqueous $\mathrm{Na}_{2} \mathrm{~S}_{2} \mathrm{O}_{3}(50 \mathrm{~mL})$, dried and concentrated in vacuo. The residue was purified by column chromatography on silica gel (EtOAc/hexane, 1:5) to give $818 \mathrm{mg}(98 \%)$ of 17 as a colorless oil: TLC, $\mathrm{R}_{f} 0.19$ (EtOAc/hexane, 1:4); $[\alpha]^{23.5}-15.2\left(c 2.82, \mathrm{CHCl}_{3}\right) ;{ }^{1} \mathrm{H}$ NMR $(300 \mathrm{MHz}) \delta 1.04(\mathrm{~s}, 9 \mathrm{H}), 1.05(\mathrm{~d}, J=5.7 \mathrm{~Hz}, 3 \mathrm{H}), 1.17(\mathrm{~s}, 3 \mathrm{H})$, $1.30-1.52(\mathrm{~m}, 4 \mathrm{H}), 1.56(\mathrm{~s}, 3 \mathrm{H}), 1.86-2.02(\mathrm{~m}, 2 \mathrm{H}), 2.73($ br s, $1 \mathrm{H}), 2.79(\mathrm{dd}, J=13.3,9.5 \mathrm{~Hz}, 1 \mathrm{H}), 3.25$ $(\mathrm{dd}, J=13.3,3.2 \mathrm{~Hz}), 3.84(\mathrm{tq}, J=5.8,5.7 \mathrm{~Hz}, 1 \mathrm{H}), 3.97(\mathrm{dq}, J=3.4,6.8 \mathrm{~Hz}, 1 \mathrm{H}), 4.17(\mathrm{~d}, J=5.8 \mathrm{~Hz}$, 2H), 4.33 (br s, 1H), $4.67(\mathrm{ddd}, J=9.5,3.2,9.4 \mathrm{~Hz}, 1 \mathrm{H}), 5.49(\mathrm{t}, J=7.2 \mathrm{~Hz}, 1 \mathrm{H}), 7.16-7.31(\mathrm{~m}, 5 \mathrm{H})$, 7.31-7.44, 7.62-7.74 (m, 10H); ${ }^{13} \mathrm{C}$ NMR (68 MHz) $\delta 10.5,13.4,19.2,23.2,25.1,27.0 \times 3,27.6,37.7$, $39.1,40.4,55.2,66.1,69.4,75.2,126.3,127.3 \times 2,127.4 \times 2,128.9 \times 3,129.4 \times 5,133.4,134.5,134.8$, 135.0, $135.8 \times 4,152.9,177.0$; IR (neat) 3520, 3080, 2940, 2860, 1780, 1600, $1460 \mathrm{~cm}^{-1}$; HRMS calcd for $\mathrm{C}_{38} \mathrm{H}_{48} \mathrm{NO}_{5} \mathrm{Si}\left(\mathrm{M}^{+}-\mathrm{H}\right) \mathrm{m} / z$ 626.3302, found 626.3293 .

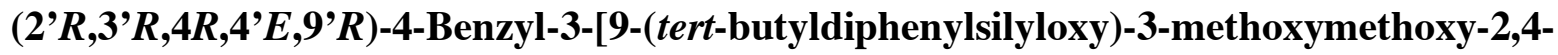

\section{dimethyl-4-decen-1-oyl]-2-oxazolidinone (18).}
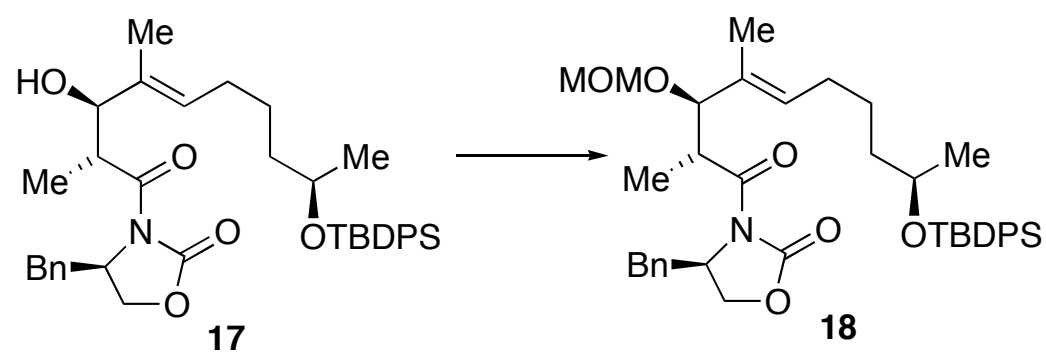

To a cooled $\left(0{ }^{\circ} \mathrm{C}\right)$, stirred solution of $\mathbf{1 7}(803 \mathrm{mg}, 1.28 \mathrm{mmol})$ in $\mathrm{CH}_{2} \mathrm{Cl}_{2}(20 \mathrm{~mL})$ were added $i$ $\operatorname{Pr}_{2} \mathrm{NEt}(2.2 \mathrm{~mL}, 13.0 \mathrm{mmol})$ and $\mathrm{MOMCl}(0.49 \mathrm{~mL}, 6.5 \mathrm{mmol})$. The mixture was heated under reflux for $10 \mathrm{~h}$, diluted with $0.2 \mathrm{M}$ aqueous $\mathrm{HCl}(40 \mathrm{~mL})$ and extracted with $\mathrm{CH}_{2} \mathrm{Cl}_{2}(15 \mathrm{~mL} \times 3)$. The 
combined organic extracts were dried and concentrated in vacuo. The residue was purified by column chromatography on silica gel (EtOAc/hexane, 1:10) to give $847 \mathrm{mg}$ (99\%) of $\mathbf{1 8}$ as a colorless oil: TLC, $\mathrm{R}_{f} 0.44$ (EtOAc/hexane, 1:4): $[\alpha]_{\mathrm{D}}^{23}+23.0\left(c\right.$ 1.88, $\left.\mathrm{CHCl}_{3}\right) ;{ }^{1} \mathrm{H}$ NMR $(300 \mathrm{MHz}) \delta 1.03(\mathrm{~s}, 9 \mathrm{H}), 1.03(\mathrm{~d}$, $J=5.6 \mathrm{~Hz}, 3 \mathrm{H}), 1.28(\mathrm{~d}, J=6.4 \mathrm{~Hz}, 3 \mathrm{H}), 1.28-1.50(\mathrm{~m}, 4 \mathrm{H}), 1.51(\mathrm{~s}, 3 \mathrm{H}), 1.90(\mathrm{dt}, J=7.1,7.5 \mathrm{~Hz}, 2 \mathrm{H})$, $2.75(\mathrm{dd}, J=13.4,9.7 \mathrm{~Hz}, 1 \mathrm{H}), 3.25(\mathrm{dd}, \mathrm{J}=13.4,3.1 \mathrm{~Hz}, 1 \mathrm{H}), 3.35(\mathrm{~s}, 3 \mathrm{H}), 3.82(\mathrm{tq}, J=5.8,5.6 \mathrm{~Hz}$, $1 \mathrm{H}), 4.00-4.12(\mathrm{~m}, 1 \mathrm{H}), 4.10-4.20(\mathrm{~m}, 1 \mathrm{H}), 4.21(\mathrm{~d}, J=7.5 \mathrm{~Hz}, 2 \mathrm{H}), 4.46(\mathrm{~d}, J=6.6 \mathrm{~Hz}, 1 \mathrm{H}), 4.46-4.54$ $(\mathrm{m}, 1 \mathrm{H}), 4.54(\mathrm{~d}, J=6.6 \mathrm{~Hz}, 1 \mathrm{H}), 5.39(\mathrm{t}, J=7.1 \mathrm{~Hz}, 1 \mathrm{H}), 7.14-7.38(\mathrm{~m}, 5 \mathrm{H}), 7.35-7.44,7.60-7.70(\mathrm{~m}$, $10 \mathrm{H}) ;{ }^{13} \mathrm{C}$ NMR $(68 \mathrm{MHz}) \delta 11.9,13.1,19.2,23.2,25.0,27.0 \times 3,27.6,37.7,39.0,40.8,55.7 \times 2,65.9$, $69.3,81.6,93.7,127.3,127.4 \times 2,127.5,128.9 \times 2,129.4 \times 3,129.5 \times 2,130.1,131.6,134.4,134.8$, 135.3, $135.8 \times 4,153.0,174.6$; IR (neat) 3080, 2930, 2860, 1780, $1700 \mathrm{~cm}^{-1}$; HRMS calcd for $\mathrm{C}_{40} \mathrm{H}_{53} \mathrm{NO}_{6} \mathrm{Si}\left(\mathrm{M}^{+}\right) \mathrm{m} / z$ 671.3642, found 671.3639.

(2S,3R,4E,9R)-9-(tert-Butyldiphenylsilyloxy)-3-methoxymethoxy-2,4-dimethyl-4-decen-1-ol (19).

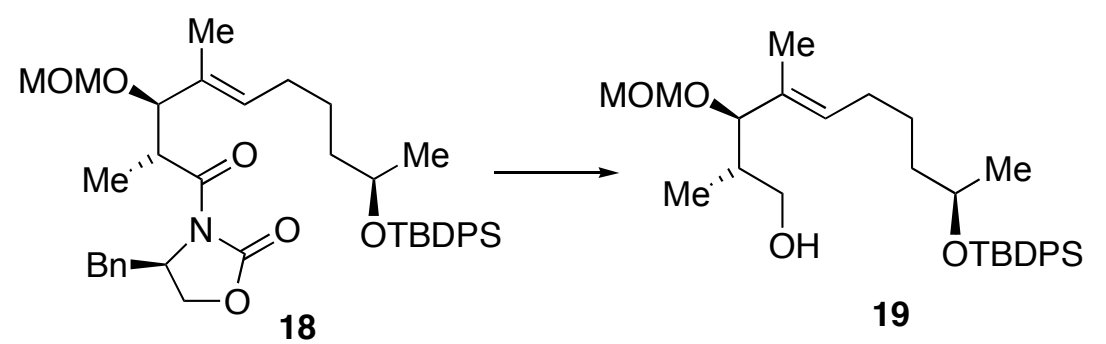

To a cooled $\left(0^{\circ} \mathrm{C}\right)$ solution of $\mathbf{1 8}(804 \mathrm{mg}, 1.20 \mathrm{mmol})$ in THF: $\mathrm{H}_{2} \mathrm{O}(3: 1,20 \mathrm{~mL})$ was added $\mathrm{NaBH}_{4}$ (225 mg, $5.95 \mathrm{mmol})$. The mixture was stirred at $0{ }^{\circ} \mathrm{C}$ for $18 \mathrm{~h}$. Then additional $\mathrm{NaBH}_{4}(90 \mathrm{mg}, 2.37$ mmol) was added. The mixture was stirred for $22 \mathrm{~h}$, quenched with saturated aqueous $\mathrm{NH}_{4} \mathrm{Cl}$. This was diluted with saturated aqueous $\mathrm{NH}_{4} \mathrm{Cl}(50 \mathrm{~mL})$ and extracted with $\mathrm{CH}_{2} \mathrm{Cl}_{2}(25 \mathrm{~mL} \times 3)$. The combined organic extracts were dried and concentrated in vacuo. The residue was purified by column chromatography on silica gel (EtOAc/hexane, 1:5) to provide $584 \mathrm{mg}(98 \%)$ of 19 as a colorless oil and $208 \mathrm{mg}(98 \%)$ of the chiral oxazolidinone was recovered. Compound 19: TLC, $\mathrm{R}_{f} 0.23$ (EtOAc/hexane, 1:4); $[\alpha]^{23.5}+79.2\left(\mathrm{c} 1.89, \mathrm{CHCl}_{3}\right) ;{ }^{1} \mathrm{H} \mathrm{NMR}(300 \mathrm{MHz}) \delta 0.96(\mathrm{~d}, J=6.8 \mathrm{~Hz}, 3 \mathrm{H}), 1.04(\mathrm{~s}, 9 \mathrm{H}), 1.05$ 
$(\mathrm{d}, J=7.1 \mathrm{~Hz}, 3 \mathrm{H}), 1.22-1.50(\mathrm{~m}, 4 \mathrm{H}), 1.51(\mathrm{~s}, 3 \mathrm{H}), 1.80-2.02(\mathrm{~m}, 3 \mathrm{H}), 1.94$ (br s, 1H), $3.39(\mathrm{~s}, 3 \mathrm{H})$, $3.42,3.52(\mathrm{dd}, J=6.8,11.1 \mathrm{~Hz}, 2 \mathrm{H}), 3.77-3.89(\mathrm{~m}, 1 \mathrm{H}), 3.83(\mathrm{~d}, J=6.8 \mathrm{~Hz}, 1 \mathrm{H}), 4.46(\mathrm{~d}, J=6.4 \mathrm{~Hz}$ $1 \mathrm{H}), 4.56(\mathrm{~d}, J=6.4 \mathrm{~Hz}, 1 \mathrm{H}), 5.34(\mathrm{t}, J=7.1 \mathrm{~Hz}, 1 \mathrm{H}), 7.32-7.46,7.64-7.72(\mathrm{~m}, 10 \mathrm{H}) ;{ }^{13} \mathrm{C}$ NMR $(68$ $\mathrm{MHz}) \delta 12.2,12.8,19.3,23.3,25.2,27.0 \times 3,27.6,37.8,39.2,55.8,65.6,69.4,83.2,93.7,127.4 \times 2$, $127.5 \times 2,129.4,129.4,132.6,134.5 \times 2,134.8,135.9 \times 4,153.0,174.6$; IR (neat) 3340, 3080, 2930, 2860, $1470 \mathrm{~cm}^{-1}$; HRMS calcd for $\mathrm{C}_{30} \mathrm{H}_{45} \mathrm{O}_{4} \mathrm{Si}\left(\mathrm{M}^{+}-\mathrm{H}\right) \mathrm{m} / z$ 497.3087, found 497.3091.

(2R,3R,4E,9R)-9-(tert-Butyldiphenylsilyloxy)-3-methoxymethoxy-2,4-dimethyl-4-decenal (20).

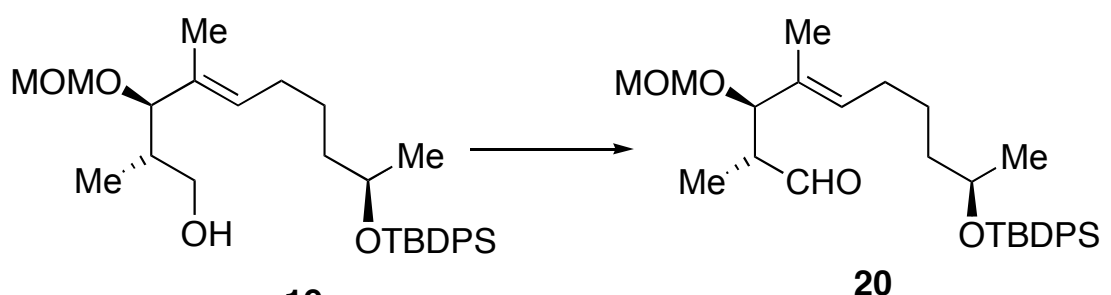

19

To a cooled $\left(0{ }^{\circ} \mathrm{C}\right)$, stirred solution of $19(216 \mathrm{mg}, 0.43 \mathrm{mmol})$ in $\mathrm{CH}_{2} \mathrm{Cl}_{2}(5 \mathrm{~mL})$ was added DessMartin periodinane $(299 \mathrm{mg}, 0.71 \mathrm{mmol})$. The mixture was stirred vigorously for $1 \mathrm{~h}$ and diluted with $20 \%$ aqueous $\mathrm{Na}_{2} \mathrm{~S}_{2} \mathrm{O}_{3}(3 \mathrm{~mL})$ and saturated aqueous $\mathrm{NaHCO}_{3}(3 \mathrm{~mL})$ at $0{ }^{\circ} \mathrm{C}$. This was stirred for 30 min, and the organic layer was separated. This was diluted with $20 \%$ aqueous $\mathrm{Na}_{2} \mathrm{~S}_{2} \mathrm{O}_{3}(7 \mathrm{~mL})$ and saturated aqueous $\mathrm{NaHCO}_{3}(7 \mathrm{~mL})$ and then extracted with $\mathrm{CH}_{2} \mathrm{Cl}_{2}(10 \mathrm{~mL} \times 3)$. The combined organic layers were dried and concentrated in vacuo to give crude $\mathbf{2 0}$, which was used for the next step without purification. In a small-scale experiment, pure 20 was obtained by column chromatographic purification on silica gel (EtOAc/hexane, 1:20) as a colorless oil: TLC, $\mathrm{R}_{f} 0.53\left(\right.$ EtOAc/hexane, 1:4); $[\alpha]^{24}+69.3(c$ 2.75, $\left.\mathrm{CHCl}_{3}\right) ;{ }^{1} \mathrm{H}$ NMR $(300 \mathrm{MHz}) \delta 1.04(\mathrm{~s}, 9 \mathrm{H}), 1.04(\mathrm{~d}, J=7.1 \mathrm{~Hz}, 3 \mathrm{H}), 1.10(\mathrm{~d}, J=6.8 \mathrm{~Hz}, 3 \mathrm{H})$, 1.24-1.50 (m, 4H), $1.50(\mathrm{~s}, 3 \mathrm{H}), 1.84-2.02(\mathrm{~m}, 3 \mathrm{H}), 2.59(\mathrm{ddq}, J=1.9,7.1,6.8 \mathrm{~Hz}, 1 \mathrm{H}), 3.35(\mathrm{~s}, 3 \mathrm{H})$, $3.83(\mathrm{tq}, J=5.8,6.0 \mathrm{~Hz}, 1 \mathrm{H}), 4.19(\mathrm{~d}, J=7.1 \mathrm{~Hz}, 1 \mathrm{H}), 4.45(\mathrm{~d}, J=6.6 \mathrm{~Hz}, 1 \mathrm{H}), 4.60(\mathrm{~d}, J=6.6 \mathrm{~Hz}, 1 \mathrm{H})$, $5.38(\mathrm{t}, J=7.1 \mathrm{~Hz}, 1 \mathrm{H}), 7.32-7.46,7.63-7.72(\mathrm{~m}, 10 \mathrm{H}), 9.62(\mathrm{~d}, J=1.9 \mathrm{~Hz}, 1 \mathrm{H}) ;{ }^{13} \mathrm{C} \mathrm{NMR}(68 \mathrm{MHz}) \delta$ $9.7,12.2,19.2,23.2,25.0,27.0 \times 3,27.5,39.0,49.1,55.7,69.3,80.5,93.2,127.4 \times 2,127.5 \times 2,129.35$, 
129.44, 130.7, 131.1, 134.5, 134.8, $135.8 \times 4,203.4$; IR (neat) 3080, 2940, 2860, 2710, 1730, 1590, $1470 \mathrm{~cm}^{-1}$; HRMS calcd for $\mathrm{C}_{26} \mathrm{H}_{35} \mathrm{O}_{4} \mathrm{Si}\left(\mathrm{M}^{+}-t\right.$-Bu) $m / z$ 439.2305, found 439.2314.

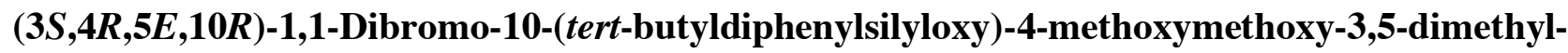
1,5-undecadiene (21).

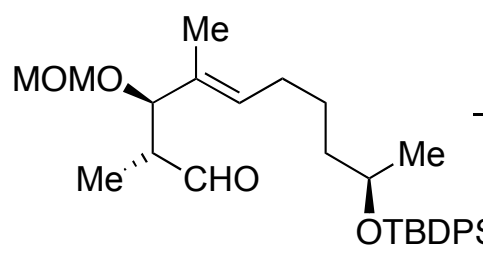

20

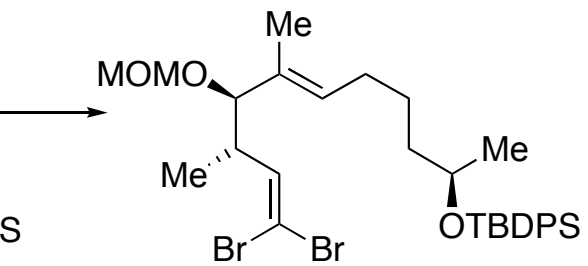

21

The following reaction was carried out under argon. To a cooled $\left(0^{\circ} \mathrm{C}\right)$, stirred solution of $\mathrm{CBr}_{4}$ (860 mg, $2.59 \mathrm{mmol})$ in $\mathrm{CH}_{2} \mathrm{Cl}_{2}(3 \mathrm{~mL})$ was added $\mathrm{PPh}_{3}(1.37 \mathrm{~g}, 5.22 \mathrm{mmol})$. This was stirred at $0{ }^{\circ} \mathrm{C}$ for $30 \mathrm{~min}$ then $\mathrm{Et}_{3} \mathrm{~N}(0.90 \mathrm{~mL}, 6.50 \mathrm{mmol})$ and a solution of crude $\mathbf{2 0}$ obtained above in $\mathrm{CH}_{2} \mathrm{Cl}_{2}(2 \mathrm{~mL})$ were added. The mixture was stirred at $0{ }^{\circ} \mathrm{C}$ for $10 \mathrm{~min}$ and quenched with saturated aqueous $\mathrm{NaHCO}_{3}$ (4 mL). This was diluted with saturated aqueous $\mathrm{NaHCO}_{3}(20 \mathrm{~mL})$ and extracted with $\mathrm{CH}_{2} \mathrm{Cl}_{2}(10 \mathrm{~mL} \times$ 3). The combined organic extracts were dried and concentrated in vacuo. The residue was purified by column chromatography on silica gel (EtOAc/hexane, 1:20) to give $230 \mathrm{mg}$ (82\% for 2 steps) of 21 as a colorless oil: TLC, $\mathrm{R}_{f} 0.63$ (EtOAc/hexane, 1:4); $[\alpha]_{\mathrm{D}}^{23.5}+82.6\left(c 2.43, \mathrm{CHCl}_{3}\right) ;{ }^{1} \mathrm{H} \mathrm{NMR}(300 \mathrm{MHz}) \delta$ $1.05(\mathrm{~s}, 9 \mathrm{H}), 1.06(\mathrm{~d}, J=6.4 \mathrm{~Hz}, 3 \mathrm{H}), 1.10(\mathrm{~d}, J=6.8 \mathrm{~Hz}, 3 \mathrm{H}), 1.30-1.44(\mathrm{~m}, 4 \mathrm{H}), 1.46(\mathrm{~s}, 3 \mathrm{H}), 1.92(\mathrm{dt}$, $J=7.1,6.7 \mathrm{~Hz}, 2 \mathrm{H}), 2.68(\mathrm{ddq}, J=8.6,9.6,6.8 \mathrm{~Hz}, 1 \mathrm{H}), 3.37(\mathrm{~s}, 3 \mathrm{H}), 3.70(\mathrm{~d}, J=8.6 \mathrm{~Hz}, 1 \mathrm{H}), 3.86(\mathrm{~d}$, $J=5.6,6.4 \mathrm{~Hz}, 1 \mathrm{H}), 4.43(\mathrm{~d}, J=6.6 \mathrm{~Hz}, 1 \mathrm{H}), 4.57(\mathrm{~d}, J=6.6 \mathrm{~Hz}, 1 \mathrm{H}), 5.27(\mathrm{t}, J=7.1 \mathrm{~Hz}, 1 \mathrm{H}), 6.08(\mathrm{~d}$, $J=9.6 \mathrm{~Hz}, 1 \mathrm{H}), 7.32-7.46,7.64-7.72(\mathrm{~m}, 10 \mathrm{H}) ;{ }^{13} \mathrm{C} \mathrm{NMR}(68 \mathrm{MHz}) \delta 11.4,15.7,19.3,23.3,25.0,27.0$ $\times 3,27.5,39.0,41.2,55.7,69.4,83.9,88.0,93.1,127.4 \times 2,127.5 \times 2,129.4,129.5,130.9,132.2,134.5$, 134.9, $135.9 \times 4$, 140.9; IR (neat) 3080, 2860, $1460 \mathrm{~cm}^{-1}$; HRMS calcd for $\mathrm{C}_{31} \mathrm{H}_{44} \mathrm{O}_{3} \mathrm{SiBr}^{81} \mathrm{Br}\left(\mathrm{M}^{+}\right) \mathrm{m} / z$ 652.1406, found 652.1421. 
undecen-1-yne (22).

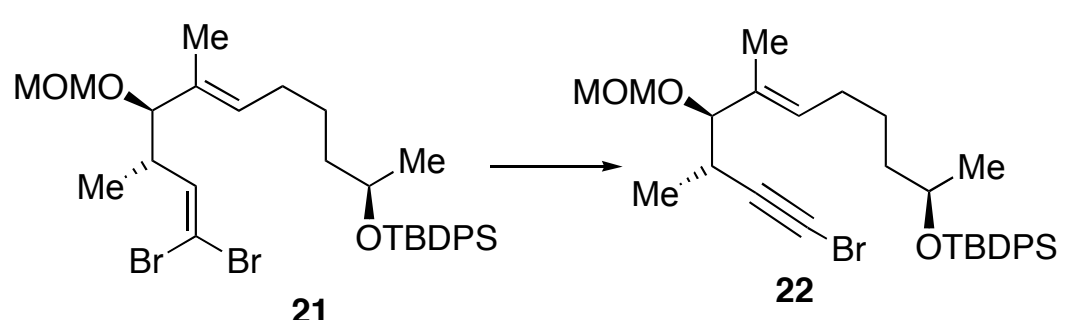

The following reaction was carried out under argon. To a cooled $\left(0{ }^{\circ} \mathrm{C}\right)$, stirred solution of $21(538$ $\mathrm{mg}, 0.82 \mathrm{mmol})$ in THF $(10 \mathrm{~mL})$ was added sodium hexamethyldisilazide $(1.0 \mathrm{M}$ solution in $\mathrm{THF}, 1.3$ $\mathrm{mL}, 1.3 \mathrm{mmol}$ ). The mixture was stirred at $0{ }^{\circ} \mathrm{C}$ for $30 \mathrm{~min}$ and quenched with saturated aqueous $\mathrm{NH}_{4} \mathrm{Cl}$. This was diluted with saturated aqueous $\mathrm{NH}_{4} \mathrm{Cl}(40 \mathrm{~mL})$ and extracted with $\mathrm{CH}_{2} \mathrm{Cl}_{2}(20 \mathrm{~mL} \times 3)$. The combined organic layers were dried and concentrated in vacuo. The residue was purified by column chromatography on silica gel (EtOAc/hexane, 1:25) to give $431 \mathrm{mg}(91 \%)$ of 22 as a colorless oil: TLC, $\mathrm{R}_{f} 0.63$ (EtOAc/hexane, 1:4): $[\alpha]^{22}{ }_{\mathrm{D}}+83.6\left(c\right.$ 1.74, $\left.\mathrm{CHCl}_{3}\right):{ }^{1} \mathrm{H}$ NMR $(300 \mathrm{MHz}) \delta 1.05(\mathrm{~s}, 9 \mathrm{H}), 1.06(\mathrm{~d}$, $J=5.8 \mathrm{~Hz}, 3 \mathrm{H}), 1.10(\mathrm{~d}, J=6.8 \mathrm{~Hz}, 3 \mathrm{H}), 1.32-1.48(\mathrm{~m}, 4 \mathrm{H}), 1.51(\mathrm{~s}, 3 \mathrm{H}), 1.95(\mathrm{~m}, 2 \mathrm{H}), 2.68(\mathrm{dq}, J=$ 8.3, $6.8 \mathrm{~Hz}, 1 \mathrm{H}), 3.36(\mathrm{~s}, 3 \mathrm{H}), 3.75(\mathrm{~d}, J=8.3 \mathrm{~Hz}, 1 \mathrm{H}), 3.86(\mathrm{dd}, J=5.3,5.8 \mathrm{~Hz}, 1 \mathrm{H}), 4.45(\mathrm{~d}, J=6.6$ $\mathrm{Hz}, 1 \mathrm{H}), 4.58(\mathrm{~d}, J=6.6 \mathrm{~Hz}, 1 \mathrm{H}), 5.36(\mathrm{t}, J=7.0 \mathrm{~Hz}, 1 \mathrm{H}), 7.30-7.46,7.62-7.74(\mathrm{~m}, 10 \mathrm{H}) ;{ }^{13} \mathrm{C} \mathrm{NMR}(68$ MHz) $\delta 11.2,17.4,19.3,23.2,24.9,27.0 \times 3,27.5,30.8,39.0,39.8,55.6,69.4,81.8,84.2,93.2,127.3 \times$ $2,127.5 \times 2,129.3,129.4,131.4,131.7,134.5,134.9,135.9 \times 4$; IR (neat) 3080, 2930, 2820, 1590, $1470 \mathrm{~cm}^{-1}$; HRMS calcd for $\mathrm{C}_{31} \mathrm{H}_{43} \mathrm{O}_{3} \mathrm{Si}\left(\mathrm{M}^{+}-\mathrm{Br}\right) \mathrm{m} / \mathrm{z}$ 491.2982, found 491.2972.

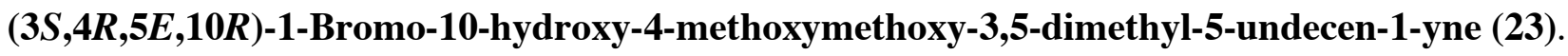<smiles>CC[C@@H](OC)[C@@H](C)OC</smiles> 
To a cooled $\left(0^{\circ} \mathrm{C}\right)$, stirred solution of $22(691 \mathrm{mg}, 1.21 \mathrm{mmol})$ in THF:pyridine $(2: 1,13 \mathrm{~mL})$ was added HF.pyridine complex $(2.6 \mathrm{~mL})$. The mixture was stirred for $18.5 \mathrm{~h}$ and quenched with saturated aqueous $\mathrm{NaHCO}_{3}(10 \mathrm{~mL})$ at $0{ }^{\circ} \mathrm{C}$. This was diluted with saturated aqueous $\mathrm{NaHCO}_{3}(40 \mathrm{~mL})$ and extracted with $\mathrm{CH}_{2} \mathrm{Cl}_{2}(25 \mathrm{~mL} \times 3)$. The combined organic layers were dried and concentrated with the aid of toluene. The residue was purified by column chromatography on silica gel (EtOAc/hexane, 1:3) to provide $391 \mathrm{mg}(97 \%)$ of $\mathbf{2 3}$ as a colorless oil: TLC, $\mathrm{R}_{f} 0.34$ (EtOAc/hexane, $\left.1: 4\right) ;[\alpha]^{28}{ }_{\mathrm{D}}+120(c 2.33$, $\left.\mathrm{CHCl}_{3}\right) ;{ }^{1} \mathrm{H} \mathrm{NMR}(300 \mathrm{MHz}) \delta 1.19(\mathrm{~d}, J=6.2 \mathrm{~Hz}, 3 \mathrm{H}), 1.27(\mathrm{~d}, J=6.8 \mathrm{~Hz}, 3 \mathrm{H}), 1.40-1.52(\mathrm{~m}, 4 \mathrm{H})$, $1.57(\mathrm{~s}, 3 \mathrm{H}), 1.61(\mathrm{br} \mathrm{s}, 1 \mathrm{H}), 2.10(\mathrm{~m}, 2 \mathrm{H}), 2.71(\mathrm{dq}, J=8.6,6.8 \mathrm{~Hz}, 1 \mathrm{H}), 3.37(\mathrm{~s}, 3 \mathrm{H}), 3.77(\mathrm{~d}, J=8.6$ $\mathrm{Hz}, 1 \mathrm{H}), 3.81(\mathrm{~m}, 1 \mathrm{H}), 4.46(\mathrm{~d}, J=6.6 \mathrm{~Hz}, 1 \mathrm{H}), 4.61(\mathrm{~d}, J=6.6 \mathrm{~Hz}, 1 \mathrm{H}), 5.44(\mathrm{t}, J=6.8 \mathrm{~Hz}, 1 \mathrm{H}) ;{ }^{13} \mathrm{C}$ NMR $(68 \mathrm{MHz}) \delta 11.2,17.4,23.4,25.5,27.4,30.8,38.8,55.6,68.0,81.8,84.3,93.2,130.9,131.5$; IR (neat) 3400, 2970, 2930, 2210, $1450 \mathrm{~cm}^{-1}$; HRMS calcd for $\mathrm{C}_{15} \mathrm{H}_{25} \mathrm{O}_{3} \mathrm{Br}\left(\mathrm{M}^{+}\right) \mathrm{m} / z$ 332.0987, found 332.0990.

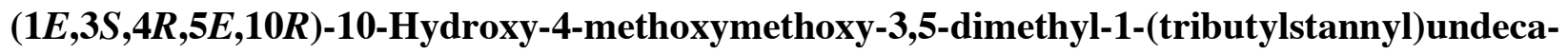
1,5-diene (5).

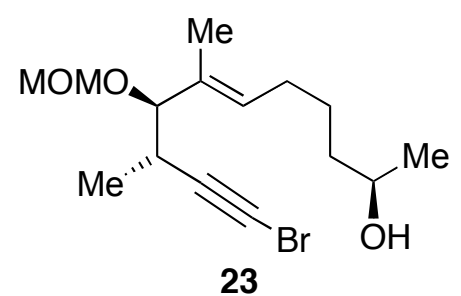

23

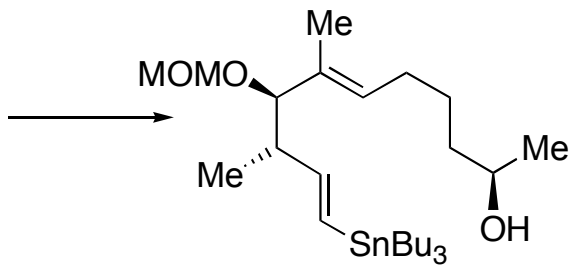

5

The following reaction was carried out under argon. To a stirred solution of 23 (353 mg, 1.06 $\mathrm{mmol})$ in THF $(7 \mathrm{~mL})$ were added $\mathrm{Pd}\left(\mathrm{PPh}_{3}\right)_{4}(123 \mathrm{mg}, 0.10 \mathrm{mmol})$ and $\mathrm{Bu}_{3} \mathrm{SnH}(0.85 \mathrm{~mL}, 3.16 \mathrm{mmol})$. The reaction mixture was stirred for $10 \mathrm{~min}$ and then concentrated in vacuo. The residue was purified by column chromatography on silica gel (EtOAc/hexane, 1:4 doped with $\left.\mathrm{Et}_{3} \mathrm{~N}\right)$ to give $504 \mathrm{mg}(87 \%)$ of 5 as a colorless oil. ${ }^{1} \mathrm{H}$ NMR analysis revealed the product contained approximately $9 \%$ of the $Z$-isomer. This mixture was used for subsequent steps without separation. Compound 5: TLC, $\mathrm{R}_{f} 0.48$ (EtOAc/hexane, 1:2); $[\alpha]_{\mathrm{D}}^{22.5}+58.7\left(\mathrm{c} 0.85, \mathrm{CHCl}_{3}\right) ;{ }^{1} \mathrm{H}$ NMR $(300 \mathrm{MHz}) \delta 0.78-0.95(\mathrm{~m}, 15 \mathrm{H}), 1.11(\mathrm{~d}$, 
$J=6.6 \mathrm{~Hz}, 3 \mathrm{H}), 1.18(\mathrm{~d}, J=6.6 \mathrm{~Hz}, 3 \mathrm{H}), 1.22-1.38,1.40-1.54(\mathrm{~m}, 12 \mathrm{H}), 1.22-1.54(\mathrm{~m}, 4 \mathrm{H}), 1.48(\mathrm{~s}, 3 \mathrm{H})$, $1.60(\mathrm{~s}, 1 \mathrm{H}), 1.88(\mathrm{~m}, 2 \mathrm{H}), 2.41(\mathrm{ddq}, J=7.1,9.0,6.2 \mathrm{~Hz}, 1 \mathrm{H}), 3.38(\mathrm{~s}, 3 \mathrm{H}), 3.63(\mathrm{~d}, J=9.0 \mathrm{~Hz}, 1 \mathrm{H})$, $3.84(\mathrm{~m}, 1 \mathrm{H}), 4.45(\mathrm{~d}, J=6.6 \mathrm{~Hz}, 1 \mathrm{H}), 4.62(\mathrm{~d}, J=6.6 \mathrm{~Hz}, 1 \mathrm{H}), 5.32(\mathrm{t}, J=6.6 \mathrm{~Hz}, 1 \mathrm{H}), 5.71(\mathrm{dd}, J=$ 7.1, $19.0 \mathrm{~Hz}, 1 \mathrm{H}), 5.87(\mathrm{~d}, J=19.0 \mathrm{~Hz}, 1 \mathrm{H}) ;{ }^{13} \mathrm{C} \mathrm{NMR}(68 \mathrm{MHz}) \delta 9.4 \times 3,11.4,13.7 \times 3,17.1,23.4$, $25.7,27.2,27.4 \times 3,29.1 \times 3,39.1,44.2,55.6,67.9,85.8,93.0,126.5,130.3,132.9,150.9 ;$ IR (neat) 3400, 2960, 1600, $1460 \mathrm{~cm}^{-1}$; HRMS calcd for $\mathrm{C}_{23} \mathrm{H}_{45} \mathrm{O}_{3} \mathrm{Sn}\left(\mathrm{M}^{+}-\mathrm{Bu}\right) \mathrm{m} / z$ 489.2391, found 489.2392.

\section{Methyl 2-(hydroxymethyl)prop-2-enoate (24).}

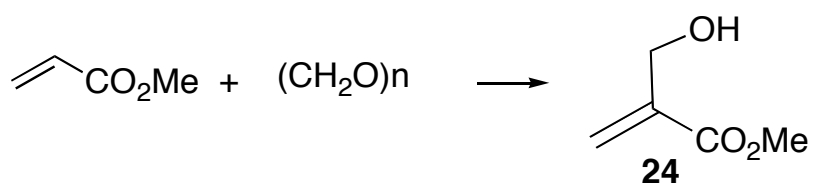

para-Formaldehyde $(3.50 \mathrm{~g}, 117 \mathrm{mmol})$ was dissolved in a mixture of 1,4-dioxane and $\mathrm{H}_{2} \mathrm{O}(1: 1,16$ $\mathrm{mL})$ and the solution was stirred for $20 \mathrm{~min}$. To this were added methyl acrylate $(21.0 \mathrm{~mL}, 23.0 \mathrm{mmol})$ and 1,4-diazabicyclo[2.2.2]octane (13.1 g, $117 \mathrm{mmol})$. The mixture was stirred for $14 \mathrm{~h}$ and diluted with saturated aqueous $\mathrm{NaCl}(200 \mathrm{~mL})$. This was extracted with $\mathrm{Et}_{2} \mathrm{O}(50 \mathrm{~mL} \times 7)$. The combined organic layers were dried and concentrated in vacuo. The residue was purified by chromatography on silica gel (EtOAc/hexane, 1:4 to 1:1) to give $24(8.33 \mathrm{~g}, 62 \%)$ as a colorless oil: TLC, $\mathrm{R}_{f} 087$ (EtOAc/hexane, 1:4); ${ }^{1} \mathrm{H}$ NMR (300 MHz) $\delta 2.37(\mathrm{t}, J=6.20 \mathrm{~Hz}, 1 \mathrm{H}), 3.80(\mathrm{~s}, 3 \mathrm{H}), 4.34(\mathrm{~d}, J=6.2 \mathrm{~Hz}, 2 \mathrm{H}), 5.87(\mathrm{~s}, 1 \mathrm{H})$, $6.27(\mathrm{~s}, 1 \mathrm{H})$.

\section{Methyl 2-(methoxymethoxymethyl)-2-propenoate (25).}

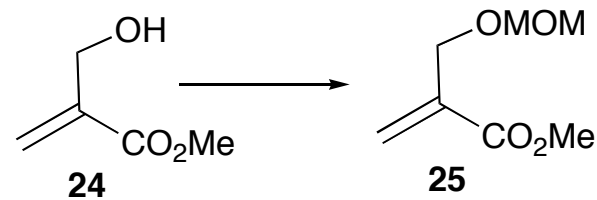

To a cooled $\left(0{ }^{\circ} \mathrm{C}\right)$, stirred solution of $24(121 \mathrm{mg}, 1.04 \mathrm{mmo})$ in $\mathrm{CH}_{2} \mathrm{Cl}_{2}(1.2 \mathrm{~mL})$ were added $i$ $\operatorname{Pr}_{2} \mathrm{NEt}(1.4 \mathrm{~mL}, 8.3 \mathrm{mmol})$ and $\mathrm{MOMCl}(0.32 \mathrm{~mL}, 4.2 \mathrm{mmol})$. The mixture was stirred for $50 \mathrm{~min}$ and 
1.0 $\mathrm{M}$ aqueous $\mathrm{HCl}(2 \mathrm{~mL})$ was added. This was diluted with $\mathrm{H}_{2} \mathrm{O}(20 \mathrm{~mL})$ and extracted with $\mathrm{CH}_{2} \mathrm{Cl}_{2}$ $(10 \mathrm{~mL} \times 3)$. The combined organic layers were dried and concentrated in vacuo. The residue was purified by column chromatography on silica gel (EtOAc/hexane, 1:50 to 1:10) to give 25 (166 mg, 75\%) as a colorless oil: TLC, $\mathrm{R}_{f} 0.62$ (EtOAc/hexane, 1;2); ${ }^{1} \mathrm{H}$ NMR (300 MHz) $\delta 3.39$ (s, 3H), 3.78 (s, 3H), $4.29(\mathrm{t}, J=1.3 \mathrm{~Hz}, 2 \mathrm{H}), 4.68(\mathrm{~s}, 2 \mathrm{H}), 5.89(\mathrm{~s}, 1 \mathrm{H}), 6.32(\mathrm{~s}, 1 \mathrm{H}) ;{ }^{13} \mathrm{C} \mathrm{NMR}(68 \mathrm{MHz}) \delta 51.6,55.3$, 65.6, 95.9, 126.3, 137.0, 166.2; IR (neat) 2950, 1730, 1645, 1442, $1395 \mathrm{~cm}^{-1}$.

Methyl 2,3-dibromo-2-[(methoxymethoxy)methyl]propanoate (26) and methyl 2,3-dibromo-2(hydroxymethyl)propanoate (27).

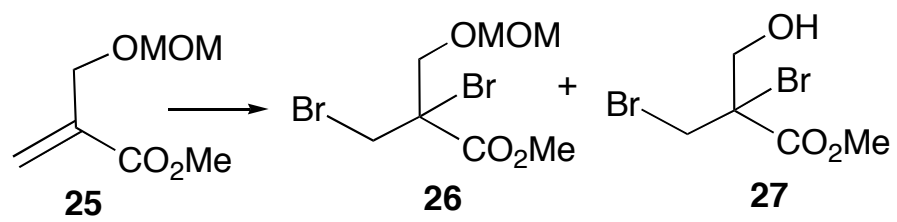

To a refluxing solution of $25(54 \mathrm{mg}, 0.38 \mathrm{mmol})$ in $\mathrm{CCl}_{4}(0.65 \mathrm{~mL})$ was added bromine $(16 \mu \mathrm{L}$, $0.32 \mathrm{mmol}$ ). After being heated under reflux for $20 \mathrm{~min}$, the solution was cooled to room temperature and $10 \%$ aqueous sodium thiosulfate $(0.5 \mathrm{~mL})$ was added. This was diluted with $\mathrm{CH}_{2} \mathrm{Cl}_{2}(10 \mathrm{~mL})$ and washed with $10 \%$ aqueous sodium thiosulfate $(10 \mathrm{~mL} \times 3)$. The organic layer was dried and concentrated in vacuo. The residue was purified by column chromatography on silica gel (EtOAc/hexane, 1:50 to 1:10) to give $72 \mathrm{mg}(66 \%)$ of $\mathbf{2 6}$ as a colorless oil and $20 \mathrm{mg}$ (22\%) of 27 as a colorless oil. Compound 26: TLC, $\mathrm{R}_{f} 0.56$ (EtOAc/hexane, 1:4); ${ }^{1} \mathrm{H}$ NMR $(300 \mathrm{MHz}) \delta 3.43(\mathrm{~s}, 3 \mathrm{H})$, $3.86(\mathrm{~s}, 3 \mathrm{H}), 4.07(2 \mathrm{~d}, J=10.3 \mathrm{~Hz}, 1 \mathrm{H} \times 2), 4.19(\mathrm{~d}, J=11.5 \mathrm{~Hz}, 2 \mathrm{H}) ;{ }^{13} \mathrm{C} \mathrm{NMR}(68 \mathrm{MHz}) \delta 33.4,53.4$, 53.8, 58.1, 68.4, 96.6, 167.8; IR (neat) 2950, 1750, $1440 \mathrm{~cm}^{-1}$. Compound 27: TLC, $\mathrm{R}_{f} 0.30$ (EtOAc/hexane, 1:4); ${ }^{1} \mathrm{H}$ NMR $(300 \mathrm{MHz}) \delta$ 2.27-3.35 (m, 1H), $3.87(\mathrm{~s}, 3 \mathrm{H}), 4.04(2 \mathrm{~d}, J=1.3 \mathrm{~Hz}, 1 \mathrm{H} \times$ 2); IR (neat) 3580, 2955, 1735, 1645, $1450 \mathrm{~cm}^{-1}$. 
Methyl 3-bromo-2-(methoxymethoxymethyl)prop-2-enoate (28).

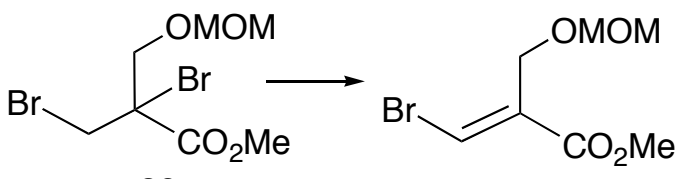

26

28

To a cooled $\left(0{ }^{\circ} \mathrm{C}\right)$, stirred solution of $\mathbf{2 6}(836 \mathrm{mg}, 2.61 \mathrm{mmol})$ in HMPA $(1.6 \mathrm{~mL})$ was added TBAF (3.9 $\mathrm{mL}, 3.9 \mathrm{mmol})$. The mixture was stirred at room temperature for $40 \mathrm{~min}$ and diluted with hexane (10 mL). This was washed with $\mathrm{H}_{2} \mathrm{O}(10 \mathrm{~mL} \times 2)$ and the aqueous layer was extracted with hexane $(10$ $\mathrm{mL} \times 2$ ). The combined organic layers were dried and concentrated in vacuo. The residue was purified by column chromatography (EtOAc/hexane, 1:30) to give $459 \mathrm{mg}(74 \%)$ of $\mathbf{2 8}$ [containing less than $2 \%$ of methyl 2-(methoxymethoxymethyl)propenoate] as a colorless oil and $142 \mathrm{mg}$ (17\%) of 26 was recovered. Compound 28: TLC, $\mathrm{R}_{f} 0.45$ (EtOAc/hexane, 1:4); ${ }^{1} \mathrm{H}$ NMR (300 MHz) $\delta 3.40(\mathrm{~s}, 3 \mathrm{H}), 3.81$ (s, 3H), $4.47(\mathrm{~s}, 2 \mathrm{H}), 4.69(\mathrm{~s}, 2 \mathrm{H}), 7.76(\mathrm{~s}, 1 \mathrm{H}) ;{ }^{13} \mathrm{C}$ NMR $(68 \mathrm{MHz}) \delta 52.4,55.4,63.0,96.3,127.4$, 134.7, 164.5; IR (neat) 2950, 1730, 1620, $1440 \mathrm{~cm}^{-1}$.

\section{Methyl (E)-2-(methoxymethoxy)methyl-5-(trimethylsilyl)pent-2-en-4-ynoate (29).}

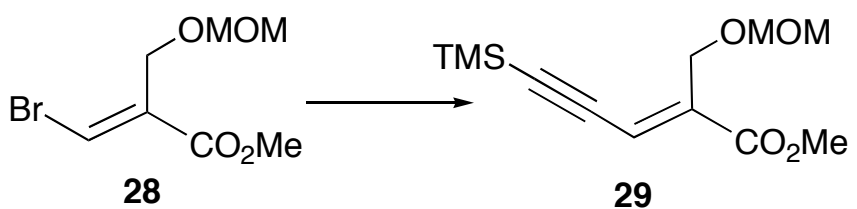

The following reaction was carried out under argon. To a cooled $\left(0{ }^{\circ} \mathrm{C}\right)$, stirred solution of 28 (429 $\mathrm{mg}, 1.79 \mathrm{mmol})$ in degassed DMF (1 mL) were added (trimethylsilyl)acetylene $(0.43 \mathrm{~mL}, 3.0 \mathrm{mmol})$, $\left(\mathrm{Ph}_{3} \mathrm{P}\right)_{4} \mathrm{Pd}(0)(104 \mathrm{mg}, 0.090 \mathrm{mmol}), i-\mathrm{Pr}_{2} \mathrm{NEt}(0.53 \mathrm{~mL}, 3.0 \mathrm{mmol})$, and $\mathrm{CuI}(68 \mathrm{mg}, 0.36 \mathrm{mmol})$. After being sturred at $0{ }^{\circ} \mathrm{C}$ for $20 \mathrm{~min}$, the mixture was diluted with saturated aqueous $\mathrm{NH}_{4} \mathrm{Cl}(40 \mathrm{~mL})$ and extracted with $\mathrm{Et}_{2} \mathrm{O}(20 \mathrm{~mL} \times 3)$. The combined organic layers were dried and concentrated in vacuo. The residue was purified by column chromatography on silica gel $\left(\mathrm{Et}_{2} \mathrm{O} / \mathrm{hexane}, 1: 10\right)$ to give $444 \mathrm{mg}(99 \%)$ of 29 as a pale yellow oil: TLC, $\mathrm{R}_{f} 0.67$ (EtOAc/hexane, 1:4); ${ }^{1} \mathrm{H}$ NMR (300 MHz) $\delta 0.23$ $(\mathrm{s}, 9 \mathrm{H}), 3.40(\mathrm{~s}, 3 \mathrm{H}), 3.80(\mathrm{~s}, 3 \mathrm{H}), 4.46(\mathrm{~s}, 2 \mathrm{H}), 4.68(\mathrm{~s}, 2 \mathrm{H}), 6.79(\mathrm{~s}, 1 \mathrm{H}) ;{ }^{13} \mathrm{C} \mathrm{NMR}(75 \mathrm{MHz}) \delta 52.2$ 
55.2, 62.7, 96.3, 99.7, 109.7, 123.7, 139.3, 166.4; IR (neat) 2950, 2890, 2140, 1730, 1615, $1440 \mathrm{~cm}^{-1}$;

HRMS calcd for $\mathrm{C}_{12} \mathrm{H}_{20} \mathrm{O}_{4} \mathrm{Si}\left(\mathrm{M}^{+}\right) \mathrm{m} / z$ 256.1131, found 256.1134 .

\section{(E)-2-(Methoxymethoxy)methyl-5-(trimethylsilyl)pent-2-en-4-yn-1-ol (30).}

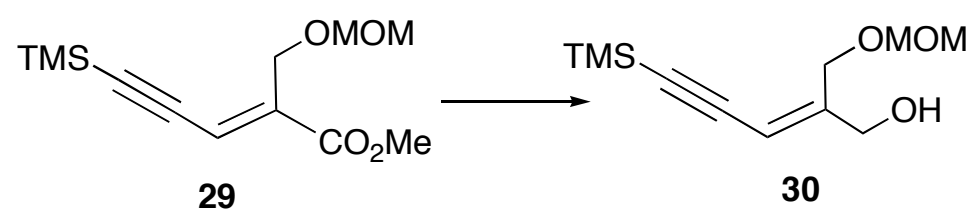

The following reaction was carried out under argon. To a cooled $\left(-78{ }^{\circ} \mathrm{C}\right)$, stirred solution of 29 (1.90 g, $7.41 \mathrm{mmol})$ in $\mathrm{CH}_{2} \mathrm{Cl}_{2}(38 \mathrm{~mL})$ was added DIBAL-H (1.01 M soution in toluene, $18.0 \mathrm{~mL}, 19.0$ mmol). After being stirred at $-78{ }^{\circ} \mathrm{C}$ for $5 \mathrm{~min}$, the mixture was quenched with $\mathrm{H}_{2} \mathrm{O}(20 \mathrm{~mL})$. The solution was warmed to room temperature, and an aqueous solution $(80 \mathrm{~mL})$ of $(+)$-tartaric acid sodium potassium salt $(13.1 \mathrm{~g})$ was added. After being stirred vigorously for $5 \mathrm{~h}$, the solution was extracted with $\mathrm{CH}_{2} \mathrm{Cl}_{2}(40 \mathrm{~mL} \times 3)$. The combined organic layers were dried and concentrated in vacuo. The residue was purified by column chromatography on silica gel (EtOAc/hexane, 1:8) to give $\mathbf{3 0}(1.20 \mathrm{~g}$, $71 \%)$ as a colorless oil: TLC, $\mathrm{R}_{f} 0.14\left(\right.$ EtOAc/hexane, 1:3); ${ }^{1} \mathrm{H}$ NMR $(300 \mathrm{MHz}) \delta 0.20(\mathrm{~s}, 9 \mathrm{H}), 2.13$ (br s, 1H), $3.41(\mathrm{~s}, 3 \mathrm{H}), 4.25(\mathrm{~d}, J=5.7 \mathrm{~Hz}, 2 \mathrm{H}), 4.42(\mathrm{~s}, 2 \mathrm{H}), 4.67(\mathrm{~s}, 2 \mathrm{H}), 5.73(\mathrm{~s}, 1 \mathrm{H}) ;{ }^{13} \mathrm{C}$ NMR $(68$ MHz) $\delta-0.2,55.4,64.5,66.1,96.4,100.6,108.6,149.9 ;$ IR (neat) 3420, 2950, 2890, 2140, 1450, 1400 $\mathrm{cm}^{-1}$; HRMS calcd for $\mathrm{C}_{11} \mathrm{H}_{20} \mathrm{O}_{3} \mathrm{Si}\left(\mathrm{M}^{+}\right) \mathrm{m} / \mathrm{z} 228.1182$, found 228.1182 .

\section{(E)-1-Bromo-2-(methoxymethoxy)methyl-5-(trimethylsilyl)pent-2-en-4-yne (31).}

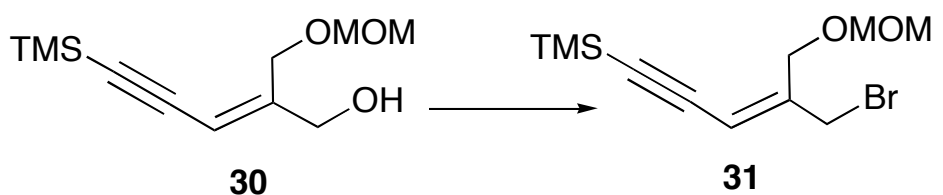

To a cooled $\left(0{ }^{\circ} \mathrm{C}\right)$, stirred solution of $\mathbf{3 0}(1.20 \mathrm{~g}, 5.25 \mathrm{mmol})$ in $\mathrm{CH}_{2} \mathrm{Cl}_{2}(24 \mathrm{~mL})$ were added $\mathrm{CBr}_{4}$ $(2.10 \mathrm{~g}, 6.30 \mathrm{mmol})$ and $\mathrm{PPh}_{3}(1.79 \mathrm{~g}, 6.82 \mathrm{mmol})$. After being stirred for $5 \mathrm{~min}$, the mixture was concentrated in vacuo. The residue was purified by column chromatography on silica gel 
(EtOAc/hexane, 1:50 to $1: 25)$ to give $31(1.35 \mathrm{~g}, 88 \%)$ as a colorless oil: TLC, $\mathrm{R}_{f} 0.43$ (EtOAc/hexane, 1:8); ${ }^{1} \mathrm{H}$ NMR (300 MHz) $\delta 0.20$ (s, 9H), 3.42 (s, 3H), 4.13 (s, 2H), 4.45 (s, 2H), 4.68 (s, 2H), 5.81 (s, $1 \mathrm{H}) ;{ }^{13} \mathrm{C}$ NMR (75 MHz) $\delta-0.3,33.2,55.4,64.2,96.4,100.1,102.8,112.5,147.1$; IR (neat) 2955, 2130, 1620, 1450, $1400 \mathrm{~cm}^{-1}$; HRMS calcd for $\mathrm{C}_{11} \mathrm{H}_{19} \mathrm{O}_{2} \mathrm{SiBr}\left(\mathrm{M}^{+}\right) \mathrm{m} / z$ 290.0338, found 290.0339.

Diethyl (E)-2-(methoxymethoxy)methyl-5-(trimethylsilyl)pent-2-en-4-ynyl phosphonate (12).

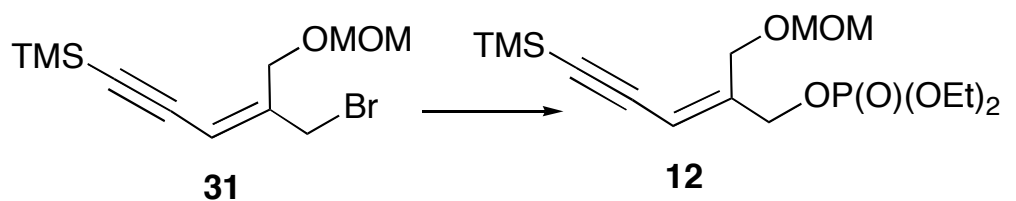

The following reaction was carried out under argon. To a solution of $\mathbf{3 1}(1.35 \mathrm{~g}, 4.63 \mathrm{mmol})$ in acetonitrile $(27 \mathrm{~mL})$ was added triethylphosphite $(1.0 \mathrm{~mL}, 6.0 \mathrm{mmol})$. The mixture was heated under reflux with stirring for $27 \mathrm{~h}$, and concentrated in vacuo with an aid of toluene. The residue was purified by column chromatography on silica gel (EtOAc/hexane, 1:1) to give $\mathbf{1 2}(1.55 \mathrm{~g}, 96 \%)$ as a colorless oil: TLC, $\mathrm{R}_{f} 0.18$ (EtOAc/hexane, 2:1); ${ }^{1} \mathrm{H}$ NMR (300 MHz) $\delta 0.19(\mathrm{~s}, 9 \mathrm{H}), 1.32(\mathrm{t}, J=7.1 \mathrm{~Hz}, 6 \mathrm{H}), 2.78(\mathrm{~d}$, $J=22.9 \mathrm{~Hz}, 2 \mathrm{H}), 3.40(\mathrm{~d}, J=0.9 \mathrm{~Hz}, 3 \mathrm{H}), 4.11,4.39(2 \mathrm{q}, J=7.1 \mathrm{~Hz}, 1 \mathrm{H} \times 2), 4.40(\mathrm{~s}, 2 \mathrm{H}), 4.65(\mathrm{~s}, 2 \mathrm{H})$, $5.66(\mathrm{~d}, J=6.0 \mathrm{~Hz}, 1 \mathrm{H}) ;{ }^{13} \mathrm{C} \mathrm{NMR}(75 \mathrm{MHz}) \delta-0.2,16.4\left(J_{\mathrm{PC}}=6.2 \mathrm{~Hz}\right), 30.6\left(J_{\mathrm{PC}}=138.0 \mathrm{~Hz}\right), 55.3$, $62.0\left(J_{\mathrm{PC}}=6.2 \mathrm{~Hz}\right), 66.5\left(J_{\mathrm{PC}}=2.5 \mathrm{~Hz}\right), 96.2,100.4\left(J_{\mathrm{PC}}=2.5 \mathrm{~Hz}\right), 100.6\left(J_{\mathrm{PC}}=6.2 \mathrm{~Hz}\right), 112.2\left(J_{\mathrm{PC}}=13.7\right.$ $\mathrm{Hz}), 142.5\left(J_{\mathrm{PC}}=12.4 \mathrm{~Hz}\right)$; IR (neat) 3480, 2960, 2130, $1440 \mathrm{~cm}^{-1}$; HRMS calcd for $\mathrm{C}_{15} \mathrm{H}_{29} \mathrm{O}_{5} \mathrm{SiP}\left(\mathrm{M}^{+}\right)$ $m / z$ 348.1522, found 348.1522.

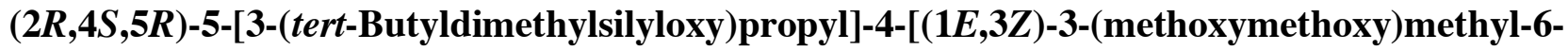
(trimethylsilyl)hexa-1,3-dien-5-ynyl]-2-phenyl-1,3-dioxane (32). 


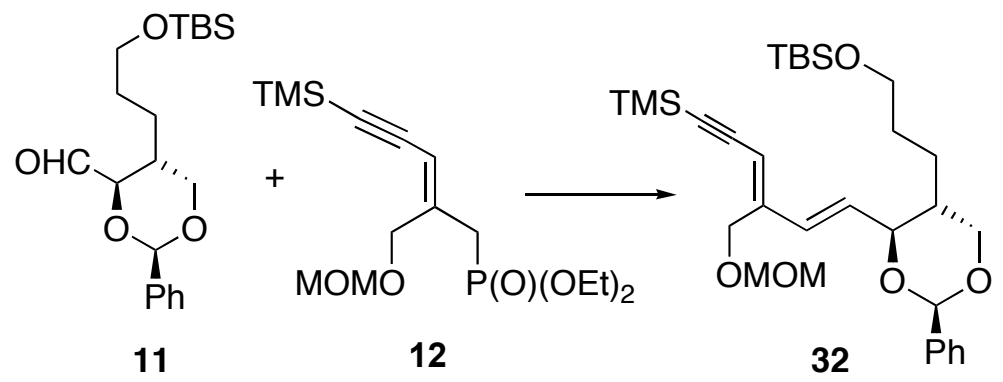

The following reaction was carried out under argon. To a cooled $\left(-78^{\circ} \mathrm{C}\right)$, stirred solution of $\mathbf{1 2}$ (1.01 g, $2.88 \mathrm{mmol})$ in THF (12 mL) was added LiHMDS (1.01 M solution in hexane, $2.8 \mathrm{~mL}, 2.9$ mmol). After being stirred at $-78{ }^{\circ} \mathrm{C}$ for $10 \mathrm{~min}$, a solution of $\mathbf{1 1}^{\mathrm{lb}}(0.890 \mathrm{~g}, 2.44 \mathrm{mmol})$ in THF $(18$ $\mathrm{mL}$ ) was added. The mixture was stirred at $-78{ }^{\circ} \mathrm{C}$ for $20 \mathrm{~min}$ and HMPA $(0.53 \mathrm{~mL}, 2.90 \mathrm{mmol})$ was added. The mixture was stirred at $-78^{\circ} \mathrm{C}$ for $30 \mathrm{~min}$, then gradually warmed to $0{ }^{\circ} \mathrm{C}$. After being stirred at $0{ }^{\circ} \mathrm{C}$ for $15 \mathrm{~min}$, the mixture was quenched with saturated aqueous $\mathrm{NH}_{4} \mathrm{Cl}(20 \mathrm{~mL})$, diluted with $\mathrm{H}_{2} \mathrm{O}$ $(20 \mathrm{~mL})$, and extracted with hexane $(20 \mathrm{~mL} \times 3)$. The combined organic layers were dried and concentrated in vacuo. The residue was purified by column chromatography on silica gel (EtOAc/hexane, 1:30 to 1:10) to give $32(1.02 \mathrm{~g}, 75 \%)$ as a colorless oil: TLC, $\mathrm{R}_{f} 0.72$ (acetone/hexane, 1;2); $[\alpha]^{26}{ }_{\mathrm{D}}-55.9\left(c\right.$ 2.06, $\left.\mathrm{CHCl}_{3}\right) ;{ }^{1} \mathrm{H}$ NMR (300 MHz) $\delta 0.04(\mathrm{~s}, 6 \mathrm{H}), 0.20(\mathrm{~s}, 9 \mathrm{H}), 0.89(\mathrm{~s}, 9 \mathrm{H}), 0.99-$ $1.11(\mathrm{~m}, 1 \mathrm{H}), 1.35-1.57(\mathrm{~m}, 3 \mathrm{H}), 1.81-1.91(\mathrm{~m}, 1 \mathrm{H}), 3.40(\mathrm{~s}, 3 \mathrm{H}), 3.52-3.64(\mathrm{~m}, 3 \mathrm{H}), 4.04(\mathrm{dd}, J=7.2$, $9.9 \mathrm{~Hz}, 1 \mathrm{H}), 4.30(\mathrm{dd}, J=4.8,11.5 \mathrm{~Hz}, 1 \mathrm{H}), 4.45,4.53(2 \mathrm{~d}, J=11.1 \mathrm{~Hz}, 1 \mathrm{H} \times 2), 5.53(\mathrm{~s}, 1 \mathrm{H}), 5.69(\mathrm{~s}$, $1 \mathrm{H}), 6.11(\mathrm{dd}, J=7.2,15.5 \mathrm{~Hz}, 1 \mathrm{H}), 6.33(\mathrm{dd}, J=7.2,15.5 \mathrm{~Hz}, 1 \mathrm{H}), 7.26-7.39(\mathrm{~m}, 3 \mathrm{H}), 7.48-7.51(\mathrm{~m}$, $2 \mathrm{H}) ;{ }^{13} \mathrm{C}$ NMR $(75 \mathrm{MHz}) \delta-5.3 \times 2,-0.2 \times 3,18.3,24.2,25.9,29.7,39.1,55.4,63.0,63.7,71.6,83.2$, 96.0, 101.2, 101.8, 103.4, 113.8, $126.2 \times 2,128.2 \times 2,128.8,130.8,132.6,138.3,146.2$; IR (neat) 2950 , 2140, 1950, 1820, 1740, $1460 \mathrm{~cm}^{-1}$; HRMS calcd for $\mathrm{C}_{31} \mathrm{H}_{50} \mathrm{O}_{5} \mathrm{Si}_{2}\left(\mathrm{M}^{+}\right) \mathrm{m} / z$ 558.3197, found 558.3174 .

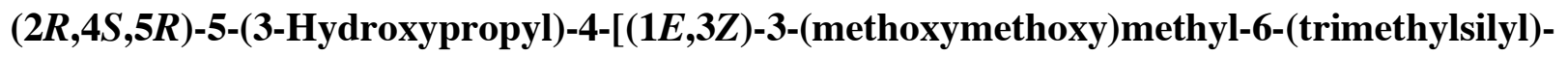
hexa-1,3-dien-5-ynyl]-2-phenyl-1,3-dioxane (33). 


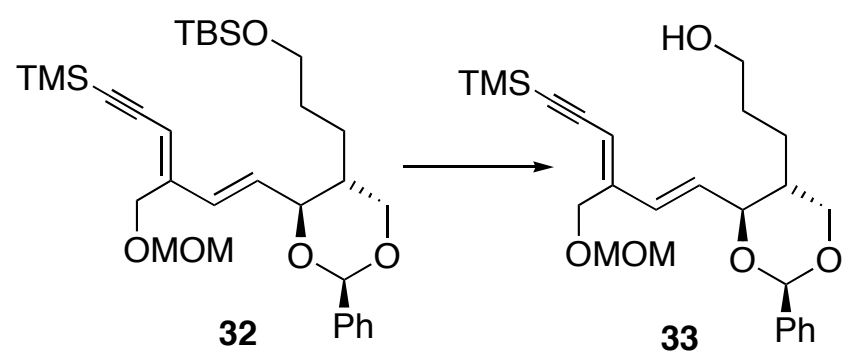

A solution of $32(981 \mathrm{mg}, 1.76 \mathrm{mmol})$ in a mixture of AcOH:THF: $\mathrm{H}_{2} \mathrm{O}(3: 2: 1 \mathrm{v} / \mathrm{v} .20 \mathrm{~mL})$ was stirred for $15 \mathrm{~h}$ and concentrated in vacuo with an aid of toluene. The residue was purified by column chromatography on silica gel (EtOAc/hexane, 2:1) to give $\mathbf{3 3}(727 \mathrm{mg}, 93 \%)$ as a colorless oil: TLC, $\mathrm{R}_{f}$ 0.40 (EtOAc: hexane, 2:1); $[\alpha]^{23}{ }_{\mathrm{D}}-70.6\left(c\right.$ 2.16, $\left.\mathrm{CHCl}_{3}\right) ;{ }^{1} \mathrm{H}$ NMR $(300 \mathrm{MHz}) \delta 0.20(\mathrm{~s}, 9 \mathrm{H}), 1.01-1.14$ (m, 1H), 1.39-1.68 (m, 3H), 1.81-1.91 (m, 1H), $3.41(\mathrm{~s}, 3 \mathrm{H}), 3.57-3.65(\mathrm{~m}, 3 \mathrm{H}), 4.09$ (ddd, $J=7.0,12.0$, $16.8 \mathrm{~Hz}, 1 \mathrm{H}), 4.30(\mathrm{dd}, J=4.5,11.4 \mathrm{~Hz}, 1 \mathrm{H}), 4.45,4.53(2 \mathrm{~d}, J=11.1 \mathrm{~Hz}, 1 \mathrm{H} \times 2), 5.54(\mathrm{~s}, 1 \mathrm{H}), 5.70(\mathrm{~s}$, $1 \mathrm{H}), 6.11(\mathrm{dd}, J=7.0,15.6 \mathrm{~Hz}, 1 \mathrm{H}), 6.34(\mathrm{~d}, J=15.6 \mathrm{~Hz}, 1 \mathrm{H}), 7.26-7.40(\mathrm{~m}, 3 \mathrm{H}), 7.49-7.51(\mathrm{~m}, 2 \mathrm{H})$; ${ }^{13} \mathrm{C}$ NMR $(68 \mathrm{MHz}) \delta-0.2 \times 3,23.7,29.7,39.0,55.1,62.3,63.7,71.6,83.2,96.1,101.1,101.7,103.6$, 114.0, $126.2 \times 2,128.3 \times 2,128.9,130.9,132.7,138.2,146.1$; IR (neat) 3450, 2950, 2120, 1950, 1890 . $1820,1460 \mathrm{~cm}^{-1}$; HRMS calcd for $\mathrm{C}_{25} \mathrm{H}_{36} \mathrm{O}_{5} \mathrm{Si}\left(\mathrm{M}^{+}\right) \mathrm{m} / \mathrm{z}$ 444.2332, found 444.2326 .

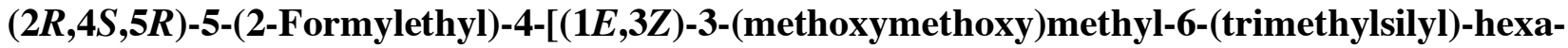
1,3-dien-5-ynyl]-2-phenyl-1,3-dioxane (34).

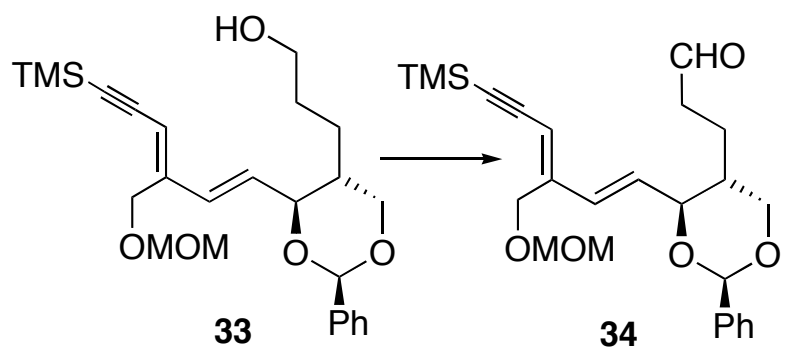

To a cooled $\left(0{ }^{\circ} \mathrm{C}\right)$, stirred solution of $\mathbf{3 3}(747 \mathrm{mg}, 1.68 \mathrm{mmol})$ in $\mathrm{CH}_{2} \mathrm{Cl}_{2}(15 \mathrm{~mL})$ was added DessMartin periodinane $(926 \mathrm{mg}, 2.18 \mathrm{mmol})$. After being warmed to room temperature, the mixture was stirred for $2 \mathrm{~h}$. Under ice-cooling, the mixture was quenched with a mixed solution of saturated aqueous $\mathrm{NaHCO}_{3}$ and $10 \%$ aqueous sodium thiosulfate $(1: 1 \mathrm{v} / \mathrm{v}, 30 \mathrm{ml})$. This was stirred for $35 \mathrm{~min}$ and 
extracted with $\mathrm{CH}_{2} \mathrm{Cl}_{2}(15 \mathrm{~mL} \times 3)$. The combined extracts were dried and concentrated in vacuo. The residue was purified by column chromatography on silica gel (EtOAc/hexane, 1:6) to give 34 (598 mg, $80 \%)$ as a colorless oil: TLC, $\mathrm{R}_{f} 0.59$ (EtOAc:hexane, $\left.1: 1\right) ;[\alpha]^{26}{ }_{\mathrm{D}}-67.4\left(c\right.$ 1.83, $\left.\mathrm{CHCl}_{3}\right) ;{ }^{1} \mathrm{H} \mathrm{NMR}(300$ $\mathrm{MHz}) \delta 0.20(\mathrm{~s}, 9 \mathrm{H}), 1.33(\mathrm{td}, J=15.1,7.5 \mathrm{~Hz}, 1 \mathrm{H}), 1.56-1.72(\mathrm{~m}, 1 \mathrm{H}), 1.74-1.91(\mathrm{~m}, 1 \mathrm{H}), 2.49(\mathrm{dt}, J=$ 0.97, $7.5 \mathrm{~Hz}, 2 \mathrm{H}) 3.40(\mathrm{~s}, 3 \mathrm{H}), 3.60(\mathrm{t}, J=11.2 \mathrm{~Hz}, 1 \mathrm{H}), 4.26(\mathrm{dd}, J=4.9,11.2 \mathrm{~Hz}, 1 \mathrm{H}), 4.05(\mathrm{dd}, J=$ 7.3, 10.0 Hz, 1H), 4.44, $4.53(2 \mathrm{~d}, J=11.1 \mathrm{~Hz}, 1 \mathrm{H} \times 2), 5.53(\mathrm{~s}, 1 \mathrm{H}), 5.71(\mathrm{~s}, 1 \mathrm{H}), 6.09(\mathrm{dd}, J=7.3,15.6$ $\mathrm{Hz}, 1 \mathrm{H}), 6.35(\mathrm{~d}, J=15.6 \mathrm{~Hz}, 1 \mathrm{H}), 7.26-7.43(\mathrm{~m}, 3 \mathrm{H}), 7.46-7.51(\mathrm{~m}, 2 \mathrm{H}), 9.75(\mathrm{t}, J=0.97 \mathrm{~Hz}, 1 \mathrm{H}) ;{ }^{13} \mathrm{C}$ NMR $(68 \mathrm{MHz}) \delta-0.3 \times 3,19.7,38.7,41.0,55.3,63.6,71.0,83.0,95.9,101.0,101.6,103.6,114.1$, $126.1 \times 2,128.1 \times 2,128.8,130.4,133.0,138.0,145.9,200.9 ; \mathrm{IR}(\mathrm{KBr}) 3020,2950,2840,2740,2120$, 1720, $1460 \mathrm{~cm}^{-1}$; HRMS calcd for $\mathrm{C}_{25} \mathrm{H}_{34} \mathrm{O}_{5} \mathrm{Si}\left(\mathrm{M}^{+}\right) \mathrm{m} / z$ 442.2176, found 442.2193.

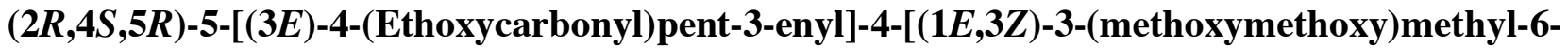
(trimethylsilyl)hexa-1,3-dien-5-ynyl]-2-phenyl-1,3-dioxane (35).

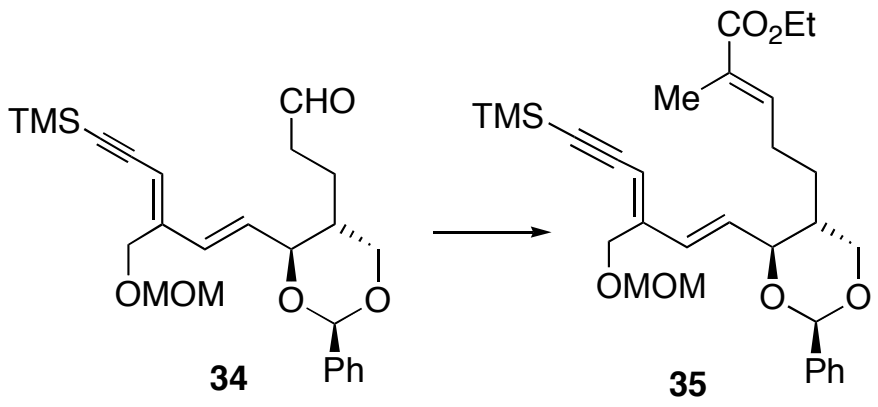

To a solution of $34(593 \mathrm{mg}, 1.34 \mathrm{mmol})$ in benzene (12 mL) was added ( $\alpha$-carboethoxyethylidene)triphenylphosphorane $(728 \mathrm{mg}, 2.01 \mathrm{mmol})$. The mixture was stirred for $15 \mathrm{~h}$ and concentrated in vacuo. The residue was purified by column chromatography on silica gel (EtOAc/hexane, 1:10 to $1: 5)$ to give $35(577 \mathrm{mg}, 82 \%)$ as a colorless oil: TLC, $\mathrm{R}_{f} 0.67$ (EtOAc/hexane, 2:1); $[\alpha]_{\mathrm{D}}^{27.5}-39.4\left(c\right.$ 1.99, $\left.\mathrm{CHCl}_{3}\right) ;{ }^{1} \mathrm{H}$ NMR $(300 \mathrm{MHz}) \delta 0.20(\mathrm{~s}, 9 \mathrm{H}), 1.08-1.22(\mathrm{~m}, 1 \mathrm{H}), 1.29(\mathrm{t}, J=$ $7.2 \mathrm{~Hz}, 3 \mathrm{H}), 1.45-1.62(\mathrm{~m}, 1 \mathrm{H}), 1.85-1.96(\mathrm{~m}, 1 \mathrm{H}), 1.82(\mathrm{~s}, 3 \mathrm{H}), 2.10-2.18(\mathrm{~m}, 2 \mathrm{H}) 3.40(\mathrm{~s}, 3 \mathrm{H}), 3.62(\mathrm{t}$, $J=11.4 \mathrm{~Hz}, 1 \mathrm{H}), 4.05(\mathrm{dd}, J=7.7,9.8 \mathrm{~Hz}, 1 \mathrm{H}), 4.19(\mathrm{q}, J=7.2 \mathrm{~Hz}, 2 \mathrm{H}), 4.31(\mathrm{dd}, J=4.2,11.4 \mathrm{~Hz}, 1 \mathrm{H})$, 4.44, $4.52(2 \mathrm{~d}, J=11.1 \mathrm{~Hz}, 1 \mathrm{H} \times 2), 4.66(\mathrm{~s}, 2 \mathrm{H}), 5.54(\mathrm{~s}, 1 \mathrm{H}), 5.71(\mathrm{~s}, 1 \mathrm{H}), 6.10(\mathrm{dd}, J=7.7,15.5 \mathrm{~Hz}$, 
$1 \mathrm{H}), 6.34(\mathrm{~d}, J=15.5 \mathrm{~Hz}, 1 \mathrm{H}), 6.66(\mathrm{t}, J=6.9 \mathrm{~Hz}, 1 \mathrm{H}), 7.26-7.40(\mathrm{~m}, 3 \mathrm{H}), 7.48-7.51(\mathrm{~m}, 2 \mathrm{H}) ;{ }^{13} \mathrm{C} \mathrm{NMR}$

$(68 \mathrm{MHz}) \delta-0.2 \times 3,12.4,14.3,25.2,26.7,38.9,55.4,60.5,63.6,71.3,83.1,96.0,101.1,101.7,103.6$ $114.0,126.1 \times 2,128.2 \times 2,128.6,128.9,130.5,133.0,138.1,140.5,146.1,167.9 ;$ IR (neat) 2950, 2350, 2130, 1720, 1650, $1460 \mathrm{~cm}^{-1}$; HRMS calcd for $\mathrm{C}_{30} \mathrm{H}_{42} \mathrm{O}_{6} \mathrm{Si}\left(\mathrm{M}^{+}\right) \mathrm{m} / z$ 526.2751 found 526.2764.

$(2 R, 4 S, 5 R)-5-[(3 E)-(5-H y d r o x y-4-m e t h y l) p e n t-3-e n y l]-4-[(1 E, 3 Z)-3-($ methoxymethoxy)methyl6-(trimethylsilyl)hexa-1,3-dien-5-ynyl]-2-phenyl-1,3-dioxane (36).

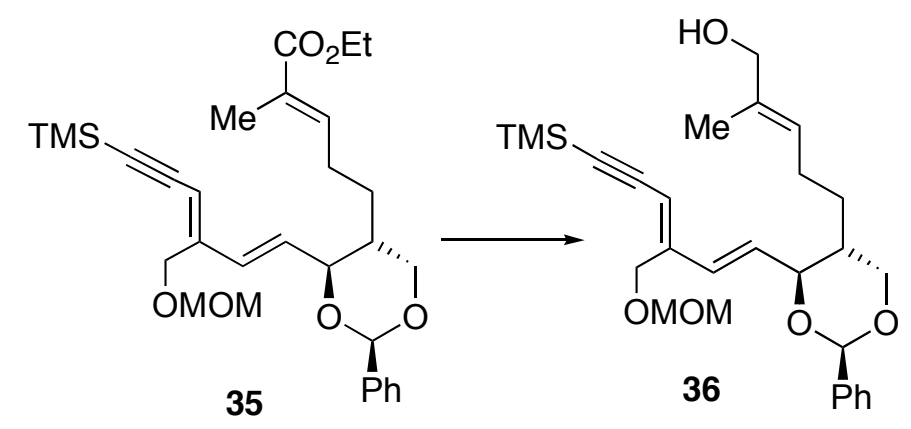

The following reaction was carried out under argon. To a cooled $\left(-78^{\circ} \mathrm{C}\right)$, stirred solution of $35(578$ $\mathrm{mg}, 1.10 \mathrm{mmol})$ in $\mathrm{CH}_{2} \mathrm{Cl}_{2}(12 \mathrm{~mL})$ was added DIBAL-H (1.01 M solution in toluene, $2.7 \mathrm{~mL}, 2.7$ mmol). After being stirred at $-78^{\circ} \mathrm{C}$ for $10 \mathrm{~min}$, the mixture was quenched with $\mathrm{H}_{2} \mathrm{O}(10 \mathrm{~mL})$. This was warmed to room temperature and an aqueous solution $(25 \mathrm{~mL})$ of (+)-tartaric acid sodium potassium salt (1.93 g) was added. After being stirred for $17 \mathrm{~h}$, this was extracted with hexane $(6 \mathrm{~mL} \times$ 3). The combined organic layers were dried and concentrated in vacuo. The residue was purified by column chromatography on silica gel (EtOAc:hexane, 1:4) to give $\mathbf{3 6}$ (502 mg, 94\%) as a colorless oil: TLC, $\mathrm{R}_{f}$ 0.45(EtOAc/hexane, 1:1); $[\alpha]^{23}{ }_{\mathrm{D}}-36.6\left(c 0.77, \mathrm{CHCl}_{3}\right) ;{ }^{1} \mathrm{H}$ NMR $(300 \mathrm{MHz}) \delta 0.20(\mathrm{~s}, 9 \mathrm{H})$, $1.02-1.14(\mathrm{~m}, 1 \mathrm{H}), 1.37-1.48(\mathrm{~m}, 2 \mathrm{H}), 1.58-1.61(\mathrm{~m}, 1 \mathrm{H}), 1.65(\mathrm{~s}, 3 \mathrm{H}), 2.02(\mathrm{dt}, J=4.5,7.5 \mathrm{~Hz}, 2 \mathrm{H})$ $3.40(\mathrm{~s}, 3 \mathrm{H}), 3.61(\mathrm{t}, J=11.2 \mathrm{~Hz}, 1 \mathrm{H}), 3.98-4.07(\mathrm{~m}, 1 \mathrm{H}), 4.19(\mathrm{q}, J=7.2 \mathrm{~Hz}, 3 \mathrm{H}), 4.32(\mathrm{dd}, J=4.5$ $11.2 \mathrm{~Hz}, 1 \mathrm{H}), 4.45,4.52(2 \mathrm{~d}, J=11.1 \mathrm{~Hz}, 1 \mathrm{H} \times 2), 4.66(\mathrm{~s}, 2 \mathrm{H}), 5.54(\mathrm{~s}, 1 \mathrm{H}), 5.70(\mathrm{~s}, 1 \mathrm{H}), 6.10(\mathrm{dd}, J=$ 7.5, 15.7 Hz, 1H), $6.33(\mathrm{~d}, J=15.7 \mathrm{~Hz}, 1 \mathrm{H}), 7.26-7.40(\mathrm{~m}, 3 \mathrm{H}), 7.48-7.51(\mathrm{~m}, 2 \mathrm{H}) ;{ }^{13} \mathrm{C} \mathrm{NMR}(68 \mathrm{MHz})$ $\delta-0.2 \times 3,13.7,24.5,27.5,38.7,55.4,63.7,68.6,71.5,76.5,83.1,96.0,101.1,101.7,113.9,124.8$, 
$126.1 \times 2,128.2 \times 2,128.8,130.8,132.7,135.6,138.2,146.1 ;$ IR (neat) $3450,2920,2130,1950,1890$ $1450 \mathrm{~cm}^{-1}$; HRMS calcd for $\mathrm{C}_{28} \mathrm{H}_{40} \mathrm{O}_{5} \mathrm{Si}\left(\mathrm{M}^{+}\right) \mathrm{m} / z$ 484.2645, found 484.2653.

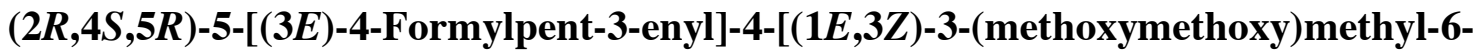

\section{(trimethylsilyl)hexa-1,3-dien-5-ynyl]-2-phenyl-1,3-dioxane (10).}

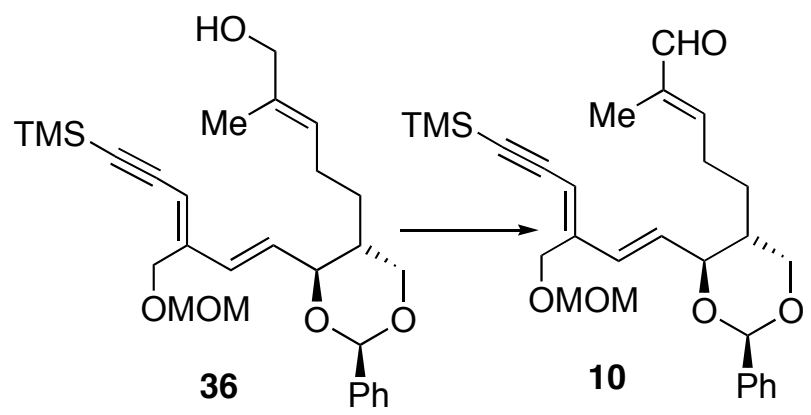

To a cooled $\left(0{ }^{\circ} \mathrm{C}\right)$, stirred solution of $36(502 \mathrm{mg}, 1.03 \mathrm{mmol})$ in $\mathrm{CH}_{2} \mathrm{Cl}_{2}(10 \mathrm{~mL})$ was added DessMartin periodinane $(571 \mathrm{mg}, 1.35 \mathrm{mmol})$. After being stirred at room temperature for $45 \mathrm{~min}$, the mixture was quenched with a mixture of saturated aqueous $\mathrm{NaHCO}_{3}$ and $20 \%$ aqueous sodium thiosulfate $(1: 1 \mathrm{v} / \mathrm{v}, 20 \mathrm{~mL})$. This was stirred for 50 min vigorously and extracted with $\mathrm{CH}_{2} \mathrm{Cl}_{2}(10 \mathrm{~mL}$ $\times 3$ ). The combined organic layers were dried and concentrated in vacuo. The residue was purified by column chromatography on silica gel (EtOAc/hexane, 1:8 to 1:4) to give $\mathbf{1 0}(460 \mathrm{mg}, 92 \%)$ as a colorless oil: TLC, $\mathrm{R}_{f} 0.36$ (EtOAc/hexane, $\left.2: 1\right) ;[\alpha]^{22}{ }_{\mathrm{D}}-35.2\left(c 0.89, \mathrm{CHCl}_{3}\right) ;{ }^{1} \mathrm{H}$ NMR $(300 \mathrm{MHz}) \delta$ $0.20(\mathrm{~s}, 9 \mathrm{H}), 1.13-1.28(\mathrm{~m}, 1 \mathrm{H}), 1.45-1.62(\mathrm{~m}, 1 \mathrm{H}), 1.70(\mathrm{~s}, 3 \mathrm{H}), 1.85-1.95(\mathrm{~m}, 1 \mathrm{H}), 2.31-2.39(\mathrm{~m}, 2 \mathrm{H})$ $3.39(\mathrm{~s}, 3 \mathrm{H}), 3.65(\mathrm{t}, J=11.4 \mathrm{~Hz}, 1 \mathrm{H}), 4.07(\mathrm{dd}, J=7.6,9.5 \mathrm{~Hz}, 1 \mathrm{H}), 4.33(\mathrm{dd}, J=4.4,11.4 \mathrm{~Hz}, 1 \mathrm{H})$, 4.43, $4.52(2 \mathrm{~d}, J=11.4 \mathrm{~Hz}, 1 \mathrm{H} \times 2), 4.65(\mathrm{~s}, 2 \mathrm{H}), 5.55(\mathrm{~s}, 1 \mathrm{H}), 5.71(\mathrm{~s}, 1 \mathrm{H}), 6.09(\mathrm{dd}, J=7.6,15.6 \mathrm{~Hz}$, 1H), $6.35(\mathrm{~d}, J=15.6 \mathrm{~Hz}, 1 \mathrm{H}), 7.26-7.40(\mathrm{~m}, 3 \mathrm{H}), 7.48-7.51(\mathrm{~m}, 2 \mathrm{H}), 9.39(\mathrm{~s}, 1 \mathrm{H}) ;{ }^{13} \mathrm{C} \mathrm{NMR}(68 \mathrm{MHz})$ $\delta-0.2 \times 3,9.3,26.0,26.5,38.9,55.4,63.6,71.2,83.1,96.0,101.1,101.5,103.9,114.3,126.1 \times 2,128.3$ $\times 2,129.0,130.3,133.2,138.0,139.8,145.9,152.9,195.0$; IR (neat) 3350, 2950, 2130, 1960, 1680, 1650, $1450 \mathrm{~cm}^{-1}$; HRMS calcd for $\mathrm{C}_{28} \mathrm{H}_{38} \mathrm{O}_{5} \mathrm{Si}\left(\mathrm{M}^{+}\right) \mathrm{m} / z$ 482.2489, found 482.2491. 


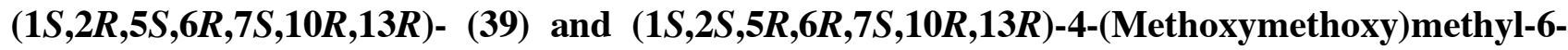
methyl-13-phenyl-5-[2-(trimethylsilyl)ethynyl]-12,14-dioxatricyclo[8.4.0.0 $\left.{ }^{2,7}\right]$-tetradec-3-ene-6carboxylic acid (40).

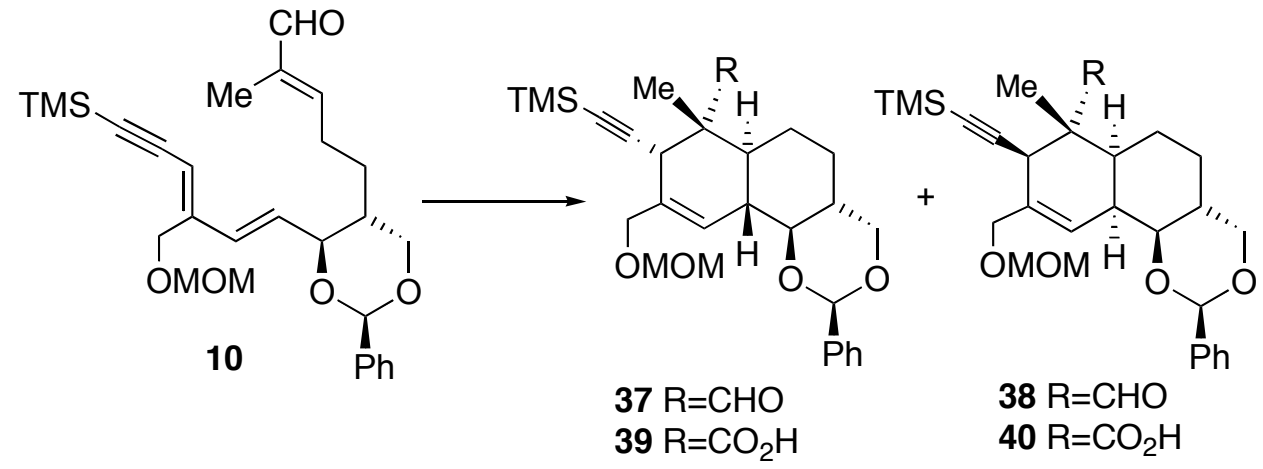

The following reaction was carried out under argon. A solution of $\mathbf{1 0}$ (460 mg, $9.53 \mathrm{mmol})$ in degassed toluene $(32 \mathrm{~mL})$ containing a piece of 2,6-di-tert-butyl-4-methylphenol was heated at $80{ }^{\circ} \mathrm{C}$ for $24 \mathrm{~h}$. After being cooled to room temperature, the solution was concentrated in vacuo. The residue was purified by column chromatography on silica gel (acetone/hexane, 1:10) to give an approximately 4:1 inseparable mixture of $\mathbf{3 7}$ and $\mathbf{3 8}$ (442 mg, 96\%) as a colorless oil: TLC, $\mathrm{R}_{f} 0.62$ (EtOAc/hexane, $3: 1) ;[\alpha]^{23.5}-13.7\left(c 2.02, \mathrm{CHCl}_{3}\right)$; for the major endo-adduct ${ }^{1} \mathrm{H}$ NMR $(270 \mathrm{MHz}) \delta 0.15(\mathrm{~s}, 9 \mathrm{H}), 0.99$ $(\mathrm{s}, 3 \mathrm{H}), 1.13-1.27(\mathrm{~m}, 2 \mathrm{H}), 1.54-1.70(\mathrm{~m}, 2 \mathrm{H}), 6 \%$ 1.86-1.90 (m, 1H), 2.12-2.16 (m, 2H), $3.02(\mathrm{~s}, 1 \mathrm{H})$, $3.36(\mathrm{~s}, 3 \mathrm{H}), 3.37-3.45(\mathrm{~m}, 1 \mathrm{H}), 3.65(\mathrm{t}, J=11.1 \mathrm{~Hz}, 1 \mathrm{H}), 3.99,4,26(2 \mathrm{~d}, J=11.7 \mathrm{~Hz}, 1 \mathrm{H} \times 2), 4.14(\mathrm{dd}$, $J=11.1 \mathrm{~Hz}, 1 \mathrm{H}), 4.59(\mathrm{~s}, 2 \mathrm{H}), 5.59(\mathrm{~s}, 1 \mathrm{H}), 6.07(\mathrm{~s}, 1 \mathrm{H}), 7.34-7.42(\mathrm{~m}, 3 \mathrm{H}), 7.50-7.53(\mathrm{~m}, 2 \mathrm{H}), 9.86(\mathrm{~s}$, $1 \mathrm{H}) ;{ }^{13} \mathrm{C} \mathrm{NMR}$ for the major endo-edduct $(75 \mathrm{MHz}) \delta-0.1 \times 3,12.5,25.3,25.5,36.7,37.3,41.1,41.2$, $48.0,55.3,68.6,71.6,83.0,90.8,95.5,101.6,102.6,124.1,126.1 \times 2,182.2 \times 2,128.8,132.6,138.4$, 206.4; IR (neat) 2930-2730, 2350, 2170, 1730, $1460 \mathrm{~cm}^{-1}$; HRMS calcd for $\mathrm{C}_{28} \mathrm{H}_{38} \mathrm{O}_{5} \mathrm{Si}\left(\mathrm{M}^{+}\right) \mathrm{m} / \mathrm{z}$ 482.2489, found 482.2501 .

To a cooled $\left(0{ }^{\circ} \mathrm{C}\right)$, stirred solution of the $4: 1$ mixture $(403 \mathrm{mg}, 0.84 \mathrm{mmol})$ in $t-\mathrm{BuOH} / \mathrm{H}_{2} \mathrm{O}(5: 1 \mathrm{v} / \mathrm{v}$, $8 \mathrm{~mL}$ ) were added 2-methyl-2-butene (0.45 mL, $4.14 \mathrm{mmol}), \mathrm{NaH}_{2} \mathrm{PO}_{4}(160 \mathrm{mg}, 1.33 \mathrm{mmol})$ and $\mathrm{NaClO}_{2}(151 \mathrm{mg}, 1.67 \mathrm{mmol})$. The mixture was stirred for $2.5 \mathrm{~h}$ and diluted with saturated aqueous $\mathrm{NH}_{4} \mathrm{Cl}(10 \mathrm{ml})$. This was extracted with $\mathrm{CH}_{2} \mathrm{Cl}_{2}(5 \mathrm{~mL} \times 3)$. The combined organic layers were dried 
and concentrated in vacuo. The residue was purified by column chromatography on silica gel (EtOAc/hexane, 1:4 to 2:1) to give $322 \mathrm{mg}(77 \%)$ of $\mathbf{3 9}$ as an amorphous solid and $77.4 \mathrm{mg}(19 \%)$ of 40 as an amorphous solid. Compound 39: TLC, $\mathrm{R}_{f} 0.30$ (EtOAc/hexane, 1:2); $[\alpha]^{24.5}{ }_{\mathrm{D}}-8.7\left(c\right.$ 1.74, $\left.\mathrm{CHCl}_{3}\right)$; ${ }^{1} \mathrm{H}$ NMR (300 MHz) $\delta 0.13(\mathrm{~s}, 9 \mathrm{H}), 1.21(\mathrm{~s}, 3 \mathrm{H}), 1.41-1.31(\mathrm{~m}, 2 \mathrm{H}), 1.63(\mathrm{~m}, 1 \mathrm{H}), 1.86(\mathrm{~m}, 1 \mathrm{H}), 1.99-$ $2.15(\mathrm{~m}, 3 \mathrm{H}), 3.22(\mathrm{~s}, 1 \mathrm{H}), 3.34-3.41(\mathrm{~m}, 1 \mathrm{H}), 3.37(\mathrm{~s}, 3 \mathrm{H}), 3.64(\mathrm{dd}, J=11,11 \mathrm{~Hz}, 1 \mathrm{H}), 4.13(\mathrm{dd}, J=$ $11,4.0 \mathrm{~Hz}, 1 \mathrm{H}), 4.13(2 \mathrm{~d}, J=12 \mathrm{~Hz}, 1 \mathrm{H} \times 2), 4.62(2 \mathrm{~d}, J=6.4 \mathrm{~Hz}, 1 \mathrm{H} \times 2), 5.58(\mathrm{~s}, 1 \mathrm{H}), 6.04(\mathrm{br}, 1 \mathrm{H})$, 7.32-7.41 (m, 3H), 7.51-7.53 (m, 2H); ${ }^{13} \mathrm{C}$ NMR $(75 \mathrm{MHz}) \delta-0.1 \times 3,15.9,25.4,26.3,38.2,39.4,41.7$, $42.2,48.9,55.3,69.5,71.8,83.2,88.7,95.6,101.5,104.0,123.7,126.1 \times 2,128.2 \times 2,128.7,132.1$, 138.5,180.8; IR (neat) 2960, 2180, 1700, $1460 \mathrm{~cm}^{-1}$; HRMS calcd for $\mathrm{C}_{28} \mathrm{H}_{38} \mathrm{O}_{6} \mathrm{Si}\left(\mathrm{M}^{+}\right) \mathrm{m} / z$ 498.2442, found 498.2438.

$(1 S, 2 R, 3 Z, 5 S, 6 R, 7 S, 10 R, 13 R)-4-(M e t h o x y m e t h o x y) m e t h y l-6-m e t h y l-13-p h e n y l-5-e t h y n y l-12,14-$ dioxatricyclo[8.4.0.0 $\left.{ }^{2,7}\right]$ tetradec-3-ene-6-carboxylic acid (41).

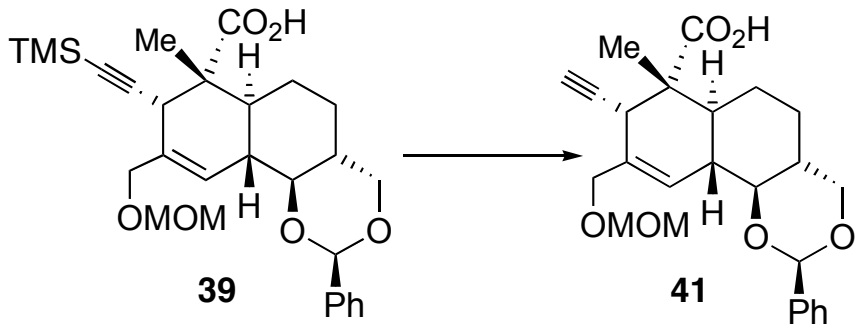

To a cooled $\left(0{ }^{\circ} \mathrm{C}\right)$, stirred solution of $39(322 \mathrm{mg}, 0.65 \mathrm{mmol})$ in THF $(6.5 \mathrm{~mL})$ was added $n$-Bu $\mathrm{Bu}_{4} \mathrm{NF}$ $(0.65 \mathrm{~mL}, 0.65 \mathrm{mmol})$. The mixture was stirred for $3 \mathrm{~h}$ and diluted with saturated aqueous $\mathrm{NH}_{4} \mathrm{Cl}(6$ $\mathrm{mL})$ and $\mathrm{H}_{2} \mathrm{O}(6 \mathrm{~mL})$. This was extracted with $\mathrm{CH}_{2} \mathrm{Cl}_{2}(5 \mathrm{~mL} \times 2)$. The combined organic layers were dried and concentrated in vacuo. The residue was purified by column chromatography on silica gel (EtOAc/hexane, 1:4 to 1:1) to give 41 (226 mg, 82\%) as a colorless oil: TLC, $\mathrm{R}_{f} 0.39$ (EtOAc/hexane, 3:1); $[\alpha]^{24.5}-17.4\left(c\right.$ 1.43, $\left.\mathrm{CHCl}_{3}\right) ;{ }^{1} \mathrm{H}$ NMR $(300 \mathrm{MHz}) \delta 1.16-1.29(\mathrm{~m}, 2 \mathrm{H}), 1.24(\mathrm{~s}, 3 \mathrm{H}), 1.66(\mathrm{~m}, 1 \mathrm{H})$, $1.89(\mathrm{~m}, 1 \mathrm{H}), 2.00-2.19(\mathrm{~m}, 3 \mathrm{H}), 2.21(\mathrm{~d}, J=2.4 \mathrm{~Hz}, 1 \mathrm{H}), 3.23($ br s, $1 \mathrm{H}), 3.94(\mathrm{~s}, 3 \mathrm{H}), 3.38(\mathrm{~m}, 1 \mathrm{H})$, $3.63(\mathrm{dd}, J=11,11 \mathrm{~Hz}, 1 \mathrm{H}), 4.14(\mathrm{dd}, J=4.0,11 \mathrm{~Hz}, 1 \mathrm{H}), 4.15(2 \mathrm{~d}, J=12 \mathrm{~Hz}, 1 \mathrm{H} \times 2), 4.62(2 \mathrm{~d}, 6.4$ 
$\mathrm{Hz}, 1 \mathrm{H} \times 2), 5.56(\mathrm{~s}, 3 \mathrm{H}), 6.07(\mathrm{br} \mathrm{s}, 1 \mathrm{H}), 7.32-7.42(\mathrm{~m}, 3 \mathrm{H}), 7.49-7.53(\mathrm{~m}, 2 \mathrm{H}) ;{ }^{13} \mathrm{C} \mathrm{NMR}(68 \mathrm{MHz}) \delta$ $15.9,25.4,26.2,38.0,38.3,41.7,42.3,48.5,55.4,69.3,71.8,72.3,82.2,83 ., 95.5,101.6,124.2,126.1 \times$ $2,128.2 \times 2,128.8,131.9,138.5,180.0 ;$ IR (neat) $3300,3000,2340,1720,1460 \mathrm{~cm}^{-1}$; HRMS calcd for $\mathrm{C}_{25} \mathrm{H}_{30} \mathrm{O}_{6}\left(\mathrm{M}^{+}\right) m / z$ 426.2040, found 426.2042.

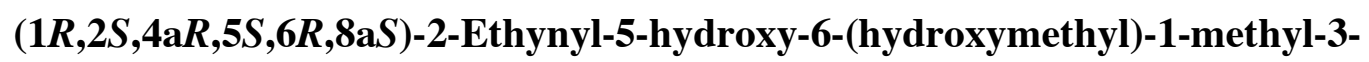
(methoxymethoxy)methyl-1,2,4a,5,6,7,8,8a-octahydronaphthalene-1-carboxylic acid (42).

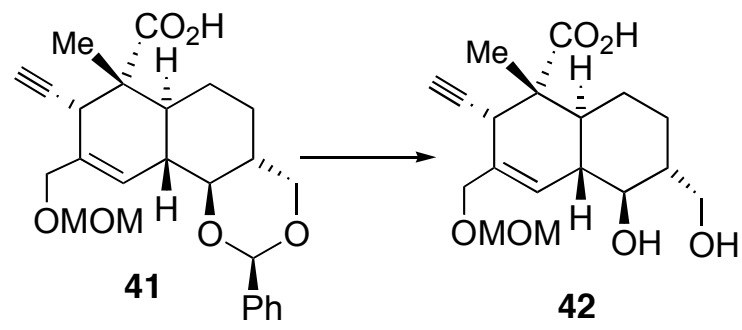

A solution of 41 (226 mg, $0.53 \mathrm{mmol})$ in $80 \%$ aqueous $\mathrm{AcOH}(10 \mathrm{~mL})$ was heated to $50{ }^{\circ} \mathrm{C}$ for $5 \mathrm{~h}$ and concentrated in vacuo with the aid of toluene to give the crude $\mathbf{4 2}$, which was used in the next step without further purification. Analytical sample was obtained by column chromatography on silica gel (EtOAc/hexane, 1:4 to acetone/hexane,1:1): TLC, $\mathrm{R}_{f} 0.44$ (acetone/hexane, 2:1); $[\alpha]_{\mathrm{D}}^{24.5}+47.1(c 0.73$, $\mathrm{MeOH}) ;{ }^{1} \mathrm{H}$ NMR (300 MHz, acetone- $\left.d_{6}\right) \delta 1.33(\mathrm{~s}, 3 \mathrm{H}), 1.09-1.25(\mathrm{~m}, 2 \mathrm{H}), 1.56(\mathrm{~m}, 1 \mathrm{H}), 1.75(\mathrm{~m}, 1 \mathrm{H})$, 2.00-2.13 (m, 3H), $2.53(\mathrm{~d}, J=2.7 \mathrm{~Hz}, 1 \mathrm{H}), 3.28(\mathrm{~s}, 3 \mathrm{H}), 3.11-3.37(\mathrm{~m}, 3 \mathrm{H}), 3.59-3.70(\mathrm{~m}, 2 \mathrm{H}), 4.08(2 \mathrm{~d}$, $J=12 \mathrm{~Hz}, 1 \mathrm{H} \times 2), 4.56(2 \mathrm{~d}, 6.4 \mathrm{~Hz}, 1 \mathrm{H} \times 2), 6.10(\mathrm{~s}, 1 \mathrm{H}) ;{ }^{13} \mathrm{C}$ NMR $\left(75 \mathrm{MHz}\right.$, acetone- $\left.d_{6}\right) \delta 16.6,27.2$, 27.9, 38.8, 46.3, 48.1, 48.6, 55.3, 64.2, 66.9, 69.8, 73.1, 76.7, 83.8, 96.0, 126.2, 132.6, 175.7; IR (neat) 2900, 2620, 2100, 1625, $1460 \mathrm{~cm}^{-1}$; HRMS calcd for $\mathrm{C}_{18} \mathrm{H}_{26} \mathrm{O}_{6}\left(\mathrm{M}^{+}\right) \mathrm{m} / z$ 338.1729, found 338.1742.

2-[(Trimethylsilyl)ethoxy]methyl

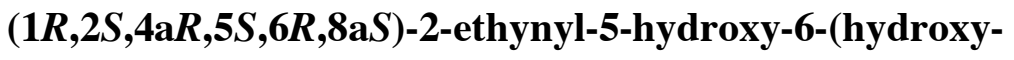
methyl)-1-methyl-3-(methoxymethoxy)methyl-1,2,4a,5,6,7,8,8a-octahydronaphthalene-1carboxylate (43). 


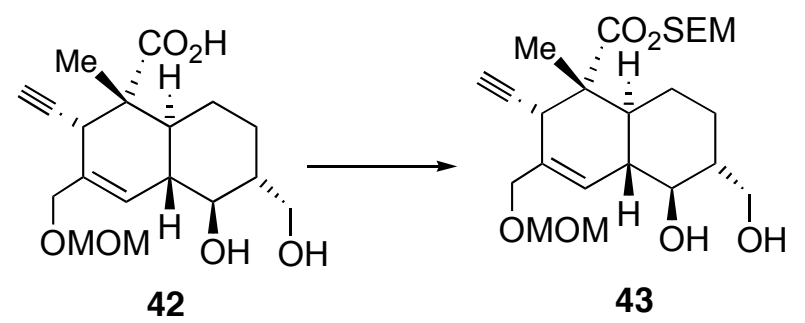

The following reaction was carried out under argon. To a cooled $\left(0{ }^{\circ} \mathrm{C}\right)$, stirred solution of crude $\mathbf{4 2}$ obtained above in THF ( $4 \mathrm{~mL})$ were added $\mathrm{Et}_{3} \mathrm{~N}(0.15 \mathrm{~mL}, 1.06 \mathrm{mmol})$ and $\mathrm{SEMCl}(94 \mathrm{~mL}, 0.53 \mathrm{mmol})$. The mixture was stirred for $2 \mathrm{~h}$ and diluted with saturated aqueous $\mathrm{NH}_{4} \mathrm{Cl}(8 \mathrm{ml})$. This was extracted with EtOAc $(4 \mathrm{~mL})$ and $\mathrm{CH}_{2} \mathrm{Cl}_{2}(4 \mathrm{~mL} \times 2)$. The combined organic layers were dried and concentrated in vacuo. The residue was purified by column chromatography on silica gel (EtOAc/hexane, 1:1 to acetone/hexane, 2:1) to provide $43\left(190 \mathrm{mg}, 76 \%\right.$ for 2 steps) as a colorless oil: TLC, $\mathrm{R}_{f} 0.56$ (EtOAc/hexane, 1:1); $[\alpha]^{24.5}{ }_{\mathrm{D}}+52.5\left(c 0.80, \mathrm{CHCl}_{3}\right) ;{ }^{1} \mathrm{H}$ NMR $(300 \mathrm{MHz}) \delta 0.02(\mathrm{~s}, 9 \mathrm{H}), 0.97(\mathrm{dd}, J=$ 7.9, $9.0 \mathrm{~Hz}, 2 \mathrm{H}), 1.05-1.25(\mathrm{~m}, 3 \mathrm{H}), 1.20(\mathrm{~s}, 3 \mathrm{H}), 1.68-1.72(\mathrm{~m}, 2 \mathrm{H}), 1.83-1.95(\mathrm{~m}, 2 \mathrm{H}), 2.11(\mathrm{~m}, 1 \mathrm{H})$, $2.15(\mathrm{~d}, J=2.7 \mathrm{~Hz}, 1 \mathrm{H}), 3.38(\mathrm{~s}, 3 \mathrm{H}), 3.36-3.44(\mathrm{~m}, 1 \mathrm{H}), 3.62-3.82(\mathrm{~m}, 5 \mathrm{H}), 4.14(2 \mathrm{~d}, J=12 \mathrm{~Hz}, 1 \mathrm{H} \times$ 2), $4.63(2 \mathrm{~d}, J=6.3 \mathrm{~Hz}, 1 \mathrm{H} \times 2), 5.33(\mathrm{~s}, 2 \mathrm{H}), 6.09(\mathrm{~s}, 1 \mathrm{H}) ;{ }^{13} \mathrm{C} \mathrm{NMR}(68 \mathrm{MHz}) \delta-1.4 \times 3,15.9,18.0$, 26.2, 26.5, 37.6, 37.9, 45.1, 46.4, 48.5, 55.3, 67.9, 68.6, 69.3, 71.9, 77.9, 82.4, 89.6, 95.5, 124.8, 131.9, 173.9; IR (neat) 3300, 2900, 2360, 1730, $1460 \mathrm{~cm}^{-1}$; HRMS calcd for $\mathrm{C}_{24} \mathrm{H}_{40} \mathrm{O}_{7} \mathrm{Si}\left(\mathrm{M}^{+}\right) \mathrm{m} / z$ 468.2543, found 468.2543 .

2-[(Trimethysilyl)ethoxy]methyl $(1 R, 2 S, 4 a R, 5 S, 6 R, 8 a S)$-2-ethynyl-5-hydroxy-6-tosyloxy-1methyl-3-(methoxymethoxy)methyl-1,2,4a,5,6,7,8,8a-octahydronaphthalene-1-carboxylate (44).

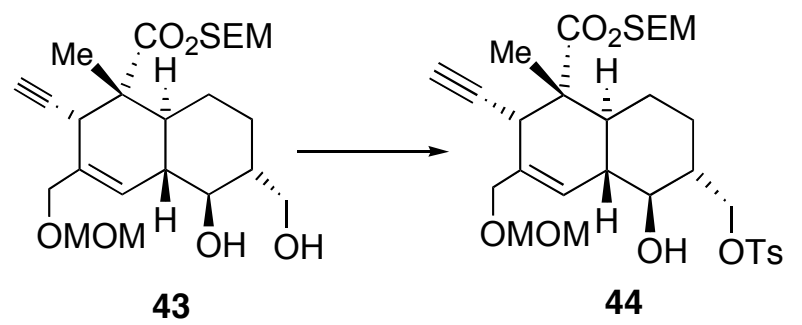

To a cooled $\left(0{ }^{\circ} \mathrm{C}\right)$, stirred solution of $\mathbf{4 3}(209 \mathrm{mg}, 0.45 \mathrm{mmol})$ in $\mathrm{CH}_{2} \mathrm{Cl}_{2}(4 \mathrm{~mL})$ were added $\mathrm{Et}_{3} \mathrm{~N}$ (0.38 mL, $2.65 \mathrm{mmol}), \mathrm{TsCl}(260 \mathrm{mg}, 1.36 \mathrm{mmol})$ and DMAP $(9.0 \mathrm{mg}, 0.07 \mathrm{mmol})$. The mixture was 
stirred for $1 \mathrm{~h}$ and diluted with saturated aqueous $\mathrm{NaHCO}_{3}(8 \mathrm{~mL})$. This was extracted with $\mathrm{Et}_{2} \mathrm{O}(4 \mathrm{~mL}$ $\times 3$ ). The combined organic layers were dried and concentrated in vacuo. The residue was purified by column chromatography on silica gel (EtOAc/hexane, 1:5 to 1:1) to 44 (267 $\mathrm{mg}, 96 \%$ ) as a colorless oil: TLC, $\mathrm{R}_{f} 0.45$ (acetone/hexane, $\left.1: 2\right) ;[\alpha]_{\mathrm{D}}^{24.5}+45.4\left(c 1.76, \mathrm{CHCl}_{3}\right) ;{ }^{1} \mathrm{H}$ NMR $(270 \mathrm{MHz}) \delta 0.02(\mathrm{~s}, 9 \mathrm{H})$, $0.97(\mathrm{dd}, J=7.9,8.9 \mathrm{~Hz}, 2 \mathrm{H}), 1.06(\mathrm{~m}, 1 \mathrm{H}), 1.17(3 \mathrm{H}, \mathrm{s}), 1.44(\mathrm{dddd}, J=12,12,12,3.6 \mathrm{~Hz}, 1 \mathrm{H}), 1.62-$ $1.84(\mathrm{~m}, 3 \mathrm{H}), 1.91(\mathrm{ddd}, J=2.1,11,11 \mathrm{~Hz}, 1 \mathrm{H}), 2.09(\mathrm{~m}, 1 \mathrm{H}), 2.16(\mathrm{~d}, J=2.6 \mathrm{~Hz}, 1 \mathrm{H}), 2.44(\mathrm{~d}, J=5.5$ $\mathrm{Hz}, 1 \mathrm{H}), 2.46$ (s, 3H), 3.17 (br s, 1H), $3.31(\mathrm{dd}, J=4.0,9.2 \mathrm{~Hz}, 1 \mathrm{H}), 3.38(\mathrm{~s}, 3 \mathrm{H}), 3.74(\mathrm{td}, J=8.5,1.5$ $\mathrm{Hz}, 2 \mathrm{H}), 4.02(\mathrm{dd}, J=2.9,2.9 \mathrm{~Hz}, 1 \mathrm{H}), 4.13(2 \mathrm{~d}, J=12 \mathrm{~Hz}, 1 \mathrm{H} \times 2), 4.32,(\mathrm{dd}, J=4.4,1.1 \mathrm{~Hz}, 1 \mathrm{H})$, $4.63(2 \mathrm{~d}, J=6.2 \mathrm{~Hz}, 1 \mathrm{H} \times 2), 5.33(2 \mathrm{~d}, J=5.6,1 \mathrm{H} \times 2), 6.06(\mathrm{br} \mathrm{s}, 1 \mathrm{H}), 7.36(\mathrm{~d}, J=8.0 \mathrm{~Hz}, 2 \mathrm{H}), 7.80$ $(\mathrm{d}, J=8 \mathrm{~Hz}, 2 \mathrm{H}) ;{ }^{13} \mathrm{C}$ NMR $(75 \mathrm{MHz}) \delta-1.5 \times 3,15.8,18.0,21.6,26.2,27.0,37.8,37.8,44.9,45.4$, $48.4,55.3,67.9,69.2,71.6,72.1,72.3,82.3,89.6,95.5,124.6,127.9 \times 2,129.9 \times 2,132.3,132.7,144.9$, 173.7; IR (neat) 2900, 2360, 1720, $1460 \mathrm{~cm}^{-1}$; HRMS calcd for $\mathrm{C}_{31} \mathrm{H}_{46} \mathrm{O}_{9} \mathrm{SiS}\left(\mathrm{M}^{+}\right) \mathrm{m} / z$ 622.2631, found 622.2652 .

\section{2-[(Trimethysilyl)ethoxy]methyl $\quad(1 R, 2 S, 4 a R, 5 S, 6 R, 8 \mathrm{a} S)-2$-ethynyl-5-hydroxy-1,6-dimethyl-3-} (methoxymethoxy)methyl-1,2,4a,5,6,7,8,8a-octahydronaphthalene-1-carboxylate (45).

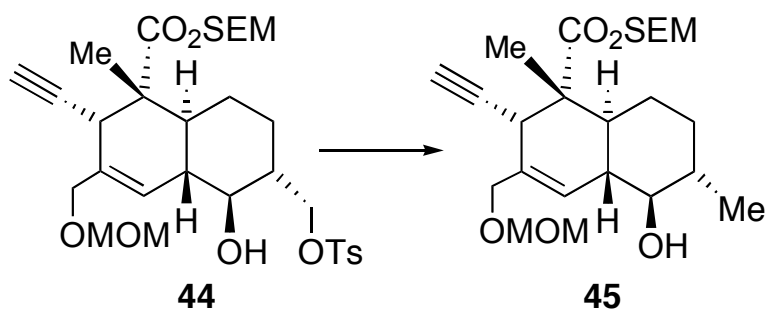

The following reaction was carried out under argon. To a heated $\left(100{ }^{\circ} \mathrm{C}\right)$, stirred solution of 44 (252 mg, $0.41 \mathrm{mmol})$ in DMSO (20 mL) was added a solution of $\mathrm{NaBH}_{4}(61 \mathrm{mg}, 1.61 \mathrm{mmol})$ in DMSO $(2 \mathrm{~mL})$. The mixture was stirred at $100{ }^{\circ} \mathrm{C}$ for $20 \mathrm{~min}$. After being cooled to $0{ }^{\circ} \mathrm{C}$, the mixture was diluted with $\mathrm{H}_{2} \mathrm{O}(10 \mathrm{~mL})$ and saturated brine $(10 \mathrm{~mL})$. This was extracted with $\mathrm{Et}_{2} \mathrm{O}(5 \mathrm{~mL} \times 3)$. The combined organic layers were dried and concentrated in vacuo. The residue was purified by column chromatography on silica gel (EtOAc/hexane, 1:4 to 1:1) to provide 45 (148 $\mathrm{mg}, 81 \%)$ as a colorless oil: 
TLC, $\mathrm{R}_{f} 0.46$ (EtOAc/hexane, 2:3); $[\alpha]^{24.5}+65.4\left(c\right.$ 2.10, $\left.\mathrm{CHCl}_{3}\right) ;{ }^{1} \mathrm{H}$ NMR $(300 \mathrm{MHz}) \delta 0.24(\mathrm{~s}, 9 \mathrm{H})$, $0.96(\mathrm{dd}, J=3.1,4.6 \mathrm{~Hz}, 2 \mathrm{H}), 1.05(\mathrm{~d}, J=6.0 \mathrm{~Hz}, 3 \mathrm{H}), 1.12(\mathrm{dd}, J=3.0,12 \mathrm{~Hz}, 1 \mathrm{H}), 1.95(\mathrm{~s}, 3 \mathrm{H}), 1.27$ $(\mathrm{dd}, J=12,3.0 \mathrm{~Hz}, 1 \mathrm{H}), 1.41-1.44(\mathrm{~m}, 1 \mathrm{H}), 1.75-1.84(\mathrm{~m}, 3 \mathrm{H}), 1.93(\mathrm{td}, J=12,2.0 \mathrm{~Hz}, 1 \mathrm{H}), 2.03(\mathrm{dd}, J$ $=12,2.6 \mathrm{~Hz}, 1 \mathrm{H}), 2.15(\mathrm{~d}, J=2.7 \mathrm{~Hz}, 1 \mathrm{H}), 2.99(\mathrm{t}, J=9.5 \mathrm{~Hz}, 1 \mathrm{H}), 3.17(\mathrm{br}, 1 \mathrm{H}), 3.38(\mathrm{~s}, 3 \mathrm{H}), 3.74$ $(\mathrm{m}, 2 \mathrm{H}), 4.14(2 \mathrm{~d}, J=12,1 \mathrm{H} \times 2), 4.63(2 \mathrm{~d}, J=6.0 \mathrm{~Hz}, 1 \mathrm{H} \times 2), 5.34(2 \mathrm{~d}, J=6.0 \mathrm{~Hz}, 1 \mathrm{H} \times 2), 6.11(\mathrm{br}$ $\mathrm{s}, 1 \mathrm{H}) ;{ }^{13} \mathrm{C} \mathrm{NMR}(75 \mathrm{MHz}) \delta-1.5 \times 3,15.9,18.0,18.5,26.9,32.9,37.9,38.5,41.0,45.3,48.6 .55 .3$, $67.9,69.3,71.9,78.5,82.5,89.5,95.5,125.5,131.8,174.0 ;$ IR (neat) $3500,3300,2950,1740,1460 \mathrm{~cm}^{-}$ ${ }^{1}$; HRMS calcd for $\mathrm{C}_{24} \mathrm{H}_{40} \mathrm{O}_{6} \mathrm{Si}\left(\mathrm{M}^{+}\right) \mathrm{m} / z$ 452.2594, found 452.2597.

\section{2-(Trimethylsilyl)ethoxymethyl $\quad(1 R, 2 S, 4 \mathrm{a} R, 5 S, 6 R, 8 \mathrm{a} S)-2-[(E)-2$-iodoethynyl]-5-hydroxy-1,6-} dimethyl-3-(methoxymethoxy)methyl-1,2,4a,5,6,7,8,8a-octahydronaphthalene-1-carboxylate (8).

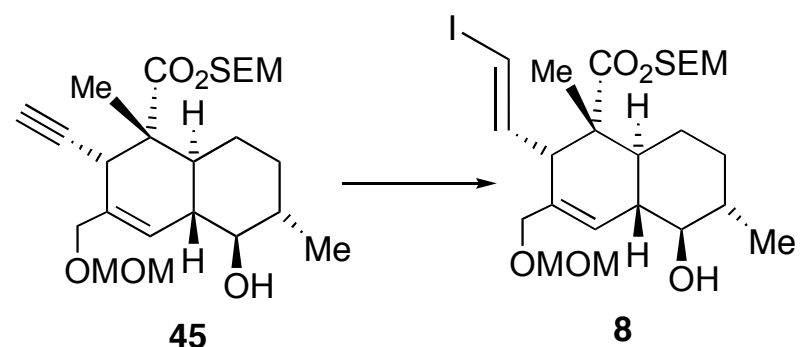

The following reaction was carried out under argon. To a cooled $\left(0{ }^{\circ} \mathrm{C}\right)$, stirred solution of $45(148$ $\mathrm{mg}, 0.33 \mathrm{mmol})$ in THF $(3 \mathrm{~mL})$ were added $\mathrm{Pd}\left(\mathrm{PPh}_{3}\right)_{2} \mathrm{Cl}_{2}(23 \mathrm{mg}, 32.8 \mu \mathrm{mol})$ and $\mathrm{Bu}_{3} \mathrm{SnH}(0.18 \mathrm{~mL}$, $0.65 \mathrm{mmol}$ ). The mixture was stirred for $5 \mathrm{~min}$ and concentrated in vacuo. The residue was purified by column chromatography on silica gel (EtOAc/hexane, 1:5) to give crude vinyl stannane (214 mg) as a colorless oil. This vinyl stannane was dissolved in $\mathrm{CH}_{2} \mathrm{Cl}_{2}(4 \mathrm{~mL})$. The solution was stirred at $0{ }^{\circ} \mathrm{C}$. To this was added a solution of $\mathrm{I}_{2}(83 \mathrm{mg}, 0.33 \mathrm{mmol})$ in $\mathrm{CH}_{2} \mathrm{Cl}_{2}(1 \mathrm{~mL})$. The mixture was stirred at $0{ }^{\circ} \mathrm{C}$ for $1 \mathrm{~h}$ and diluted with $20 \% \mathrm{Na}_{2} \mathrm{~S}_{2} \mathrm{O}_{3}(16 \mathrm{~mL})$. This was stirred for 5 min and extracted with $\mathrm{CH}_{2} \mathrm{Cl}_{2}(6$ $\mathrm{mL} \times 3$ ). The combined organic layers were dried and concentrated in vacuo. The residue was purified by column chromatography on silica gel (doped with $\mathrm{Et}_{3} \mathrm{~N}, \mathrm{EtOAc} / \mathrm{hexane}, 1: 5$ ) to give 8 (140 mg, $74 \%$ for 2 steps) as a colorless oil: TLC, $\mathrm{R}_{f} 0.34$ (EtOAc/hexane, $2: 3$ ); $[\alpha]^{24.5}{ }_{\mathrm{D}}+126.3$ (c 2.18, $\left.\mathrm{CHCl}_{3}\right) ;{ }^{1} \mathrm{H}$ 
NMR (300 MHz) $\delta 0.04(\mathrm{~s}, 9 \mathrm{H}), 0.99(\mathrm{dd}, J=8.4,8.1 \mathrm{~Hz}, 2 \mathrm{H}), 1.05(\mathrm{~d}, J=6.0 \mathrm{~Hz}, 3 \mathrm{H}), 1.18(\mathrm{~s}, 3 \mathrm{H})$, 1.20-1.27 (m, 2H), 1.30-1.64 (m, 2H), $1.72(\mathrm{~m}, 5), 2.75(\mathrm{~d}, J=10 \mathrm{~Hz}, 1 \mathrm{H}), 2.94(\mathrm{dd}, J=9.0,9.0 \mathrm{~Hz}$, $1 \mathrm{H}), 3.56(\mathrm{~s}, 3 \mathrm{H}), 3.72(\mathrm{t}, J=8.2 \mathrm{~Hz}, 2 \mathrm{H}), 3.91(2 \mathrm{~d}, J=12 \mathrm{~Hz}, 1 \mathrm{H} \times 2), 4.59(2 \mathrm{~d}, J=6.0 \mathrm{~Hz}, 1 \mathrm{H} \times 2)$, $5.26(2 \mathrm{~d}, J=6.0 \mathrm{~Hz}, 1 \mathrm{H} \times 2), 6.08(\mathrm{~d}, J=14 \mathrm{~Hz}, 1 \mathrm{H}), 6.16(\mathrm{br} \mathrm{s}, 1 \mathrm{H}), 6.34(\mathrm{dd}, J=10,14 \mathrm{~Hz}, 1 \mathrm{H}) ;{ }^{13} \mathrm{C}$ $\operatorname{NMR}(68 \mathrm{MHz}) \delta-1.4 \times 3,16.2,18.1,18.5,27.0,33.1,38.1,41.0,45.2,48.9,53.0,55.3,68.2,69.2$, 77.1, 78.8, 89.9, 95.3, 126.2, 132.0, 144.9, 174.4; IR (neat) 3450, 2950, 1740, 1600, $1460 \mathrm{~cm}^{-1}$; HRMS calcd for $\mathrm{C}_{24} \mathrm{H}_{41} \mathrm{O}_{6} \mathrm{SiI}\left(\mathrm{M}^{+}\right) \mathrm{m} / z$ 580.1717, found 580.1732.

\section{2-(Trimethylsilyl)ethoxymethyl $(1 R, 2 S, 4 a R, 5 R, 6 S, 8 a S)-5-h y d r o x y-2-[(1 E, 3 E, 5 S, 6 R, 7 E, 12 R)-12-$} hydroxy-6-methoxymethoxy-5,7-dimethyltrideca-1,3,7-trienyl]-1,3,6-trimethyl-1,2,4a,5,6,7,8,8aoctahydronaphthalene-1-carboxylate (46).

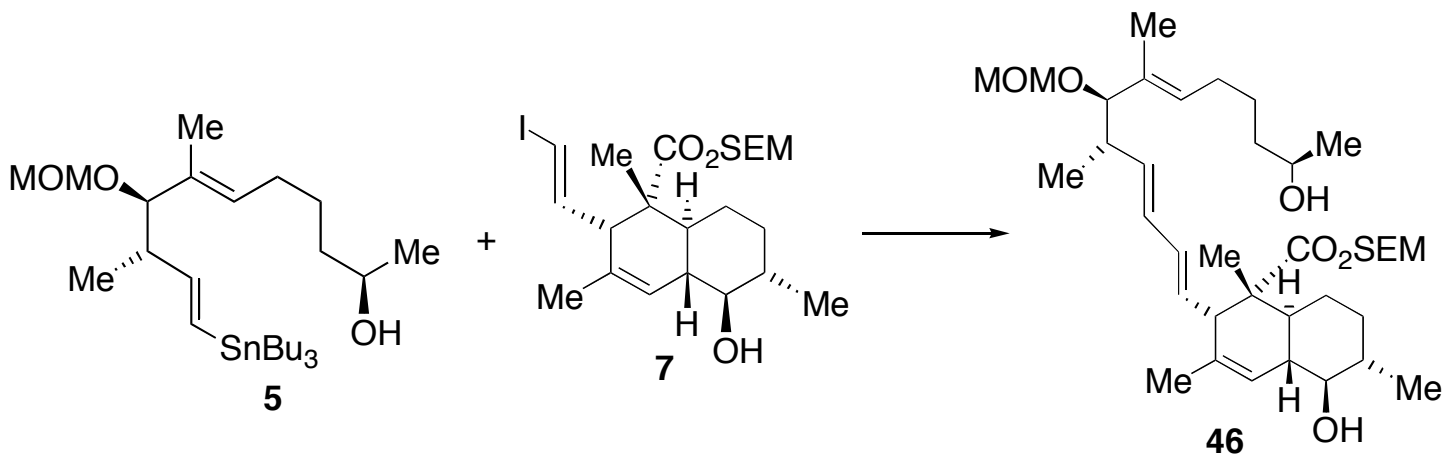

The following reaction was carried out under argon. To a stirred solution of 5 (55.4 mg, $102 \mathrm{mmol})$ and $7^{1 \mathrm{~b}}(38.5 \mathrm{mg}, 74.0 \mathrm{mmol})$ in degassed DMF $(1 \mathrm{~mL})$ was added a solution of $\operatorname{Pd}_{2}(\mathrm{dba})_{3}(3.5 \mathrm{mg}, 3.8$ $\mathrm{mmol}), \mathrm{AsPh}_{3}(9.3 \mathrm{mg}, 30 \mathrm{mmol})$ and $\mathrm{CuI}(2.9 \mathrm{mg}, 15 \mathrm{mmol})$ in degassed DMF $(0.25 \mathrm{~mL})$. The mixture was stirred for $45 \mathrm{~min}$ and then heated at $60{ }^{\circ} \mathrm{C}$ for $2 \mathrm{~h}$. After being cooled to room temperature, the mixture was diluted with saturated aqueous $\mathrm{NaHCO}_{3}(20 \mathrm{~mL})$ and extracted with $\mathrm{Et}_{2} \mathrm{O}(10 \mathrm{~mL} \times 3)$. The combined organic layers were dried and concentrated with the aid of toluene. The residue was purified by column chromatography on silica gel (EtOAc/hexane, 1:5 to 1:3) to give $35.8 \mathrm{mg}$ (75\%) of $\mathbf{4 6}$ as a colorless oil: TLC, $\mathrm{R}_{f} 0.66($ EtOAc/hexane, $2: 1) ;[\alpha]_{\mathrm{D}}^{26.5}+216\left(c 0.55, \mathrm{CHCl}_{3}\right) ;{ }^{1} \mathrm{H} \mathrm{NMR}(300 \mathrm{MHz}) \delta$ $0.03(\mathrm{~s}, 9 \mathrm{H}), 0.96(\mathrm{t}, J=8.3 \mathrm{~Hz}, 2 \mathrm{H}), 1.05(\mathrm{~d}, J=6.4 \mathrm{~Hz}, 3 \mathrm{H}), 1.10(\mathrm{~d}, J=6.6 \mathrm{~Hz}, 3 \mathrm{H}), 1.16(\mathrm{~s}, 3 \mathrm{H})$, 
$1.18(\mathrm{~d}, J=6.2 \mathrm{~Hz}, 3 \mathrm{H}), 1.32-1.51(\mathrm{~m}, 5 \mathrm{H}), 1.49(\mathrm{~s}, 3 \mathrm{H}), 1.62(\mathrm{~s}, 3 \mathrm{H}), 1.60-1.83(\mathrm{~m}, 6 \mathrm{H}), 1.96-2.09(\mathrm{~m}$,

2H), $2.35(\mathrm{~d}, J=9.6 \mathrm{~Hz}, 1 \mathrm{H}), 2.30-2.47(\mathrm{~m}, 1 \mathrm{H}), 2.91(\mathrm{~m}, 1 \mathrm{H}), 3.37(\mathrm{~s}, 3 \mathrm{H}), 3.57(\mathrm{~d}, J=9.0 \mathrm{~Hz}, 1 \mathrm{H})$, $3.68(\mathrm{t}, J=8.3 \mathrm{~Hz}, 2 \mathrm{H}), 3.78(\mathrm{~m}, 1 \mathrm{H}), 4.44,4.60(2 \mathrm{~d}, J=6.6 \mathrm{~Hz}, 1 \mathrm{H} \times 2), 5.10,5.21(2 \mathrm{~d}, J=6.0 \mathrm{~Hz}, 1 \mathrm{H}$ $\times 2), 5.28(\mathrm{~m}, 1 \mathrm{H}), 5.29(\mathrm{t}, J=6.8 \mathrm{~Hz}, 1 \mathrm{H}), 5.44(\mathrm{~m}, 1 \mathrm{H}), 5.76(\mathrm{~s}, 1 \mathrm{H}), 5.82-5.98(\mathrm{~m}, 2 \mathrm{H}) ;{ }^{13} \mathrm{C}$ NMR $(68$ $\mathrm{MHz}) \delta-1.4 \times 3,11.3,16.5,16.8,18.0,18.7,22.6,23.5,25.6,27.0,27.4,33.3,37.7,39.0,40.8 .45 .5$, $49.7,54.3,55.6,67.8,67.9,79.5,85.8,89.5,93.0,121.0,128.9,130.6,131.3,132.4,132.6,133.0,135.6$, 175.1; IR (neat) $3420,2950,2930,2880,1730,1710,1460 \mathrm{~cm}^{-1}$; HRMS calcd for $\mathrm{C}_{37} \mathrm{H}_{64} \mathrm{O}_{7} \mathrm{Si}\left(\mathrm{M}^{+}\right) \mathrm{m} / z$ 648.4421, found 648.4419.

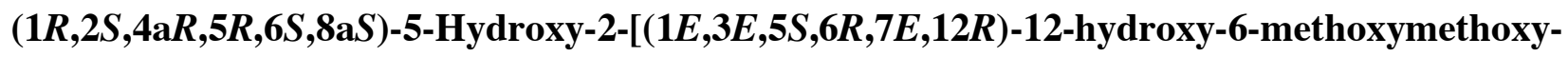
5,7-dimethyltrideca-1,3,7-trienyl]-1,3,6-trimethyl-1,2,4a,5,6,7,8,8a-octahydronaphthalene-1carboxylic acid (47).
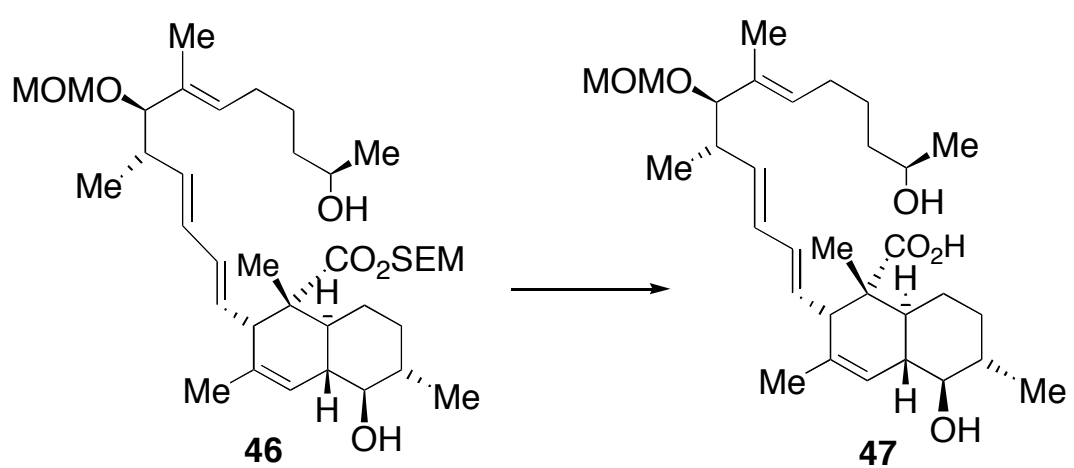

To a cooled $\left(0{ }^{\circ} \mathrm{C}\right)$, stirred solution of $46(35.5 \mathrm{mg}, 54.7 \mathrm{mmol})$ in $\mathrm{THF}(1 \mathrm{~mL})$ was added HF·pyridine complex $(0.2 \mathrm{~mL})$. The mixture was stirred for $21 \mathrm{~h}$ and quenched with saturated aqueous $\mathrm{NaHCO}_{3}(4 \mathrm{~mL})$ at $0{ }^{\circ} \mathrm{C}$. This was diluted with saturated aqueous $\mathrm{NaHCO}_{3}(16 \mathrm{~mL})$ and extracted with $\mathrm{CH}_{2} \mathrm{Cl}_{2}(20 \mathrm{~mL} \times 3)$. The combined organic layers were dried and concentrated with the aid of toluene. The residue was purified by column chromatography on silica gel (EtOAc/hexane, 1:3) to give $24.3 \mathrm{mg}$ $(86 \%)$ of 47 as a colorless oil: TLC, $\mathrm{R}_{f} 0.51$ (EtOAc/hexane, $\left.2: 1\right) ;[\alpha]^{25}{ }_{\mathrm{D}}+198\left(c 1.02, \mathrm{CHCl}_{3}\right) ;{ }^{1} \mathrm{H}$ NMR $(300 \mathrm{MHz}) \delta 1.04(\mathrm{~d}, J=6.4 \mathrm{~Hz}, 3 \mathrm{H}), 1.14(\mathrm{~d}, J=6.8 \mathrm{~Hz}, 3 \mathrm{H}), 1.15(\mathrm{~s}, 3 \mathrm{H}), 1.19(\mathrm{~d}, J=6.2 \mathrm{~Hz}, 3 \mathrm{H})$, 1.31-1.49 (m, 5H), $1.51(\mathrm{~s}, 3 \mathrm{H}), 1.63(\mathrm{~s}, 3 \mathrm{H}), 1.55-1.83(\mathrm{~m}, 6 \mathrm{H}), 1.93-2.13(\mathrm{~m}, 2 \mathrm{H}), 2.38(\mathrm{~d}, J=9.4 \mathrm{~Hz}$ 
$1 \mathrm{H}), 2.32-2.49(\mathrm{~m}, 1 \mathrm{H}), 2.91(\mathrm{~m}, 1 \mathrm{H}), 3.37(\mathrm{~s}, 3 \mathrm{H}), 3.57(\mathrm{~d}, J=9.4 \mathrm{~Hz}, 1 \mathrm{H}), 3.77-3.92(\mathrm{~m}, 1 \mathrm{H}), 4.44$ $4.60(2 \mathrm{~d}, J=6.7 \mathrm{~Hz}, 1 \mathrm{H} \times 2), 5.28-5.52(\mathrm{~m}, 3 \mathrm{H}), 5.75(\mathrm{~s}, 1 \mathrm{H}), 5.85-6.02(\mathrm{~m}, 2 \mathrm{H}) ;{ }^{13} \mathrm{C} \mathrm{NMR}(68 \mathrm{MHz}) \delta$ $11.3,16.6,16.7,18.7,22.8,23.1,25.3,26.8,27.1,33.5,37.6,38.3,38.4,40.8,45.7,49.0,53.9,55.6$, $68.3,79.5,86.2,92.9,120.9,129.3,130.8,131.5,132.4,132.6,133.2,134.7,178.3$; IR (neat) 3420, $2970,2930,2880,1710,1690,1460,1450 \mathrm{~cm}^{-1}$.

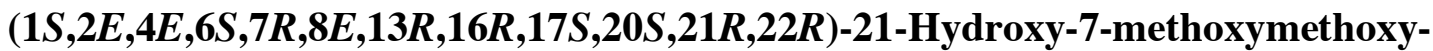

\section{6,8,13,16,20,24-hexamethyl-14-oxatricyclo[14.8.0.0 $\left.{ }^{17,22}\right]$ tetracosa-2,4,8,23-tetraene-15-one (48).}
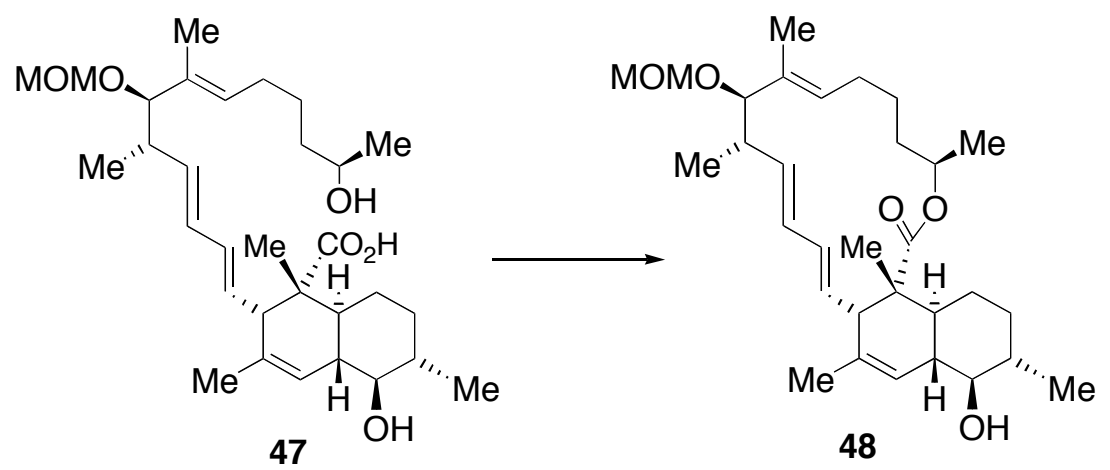

A solution of $47(20.2 \mathrm{mg}, 38.9 \mu \mathrm{mol})$ in $\mathrm{MeCN}(24 \mathrm{~mL})$ was added to a refluxing solution of 2chloro-1-methylpyridinium iodide $(50.1 \mathrm{mg}, 0.196 \mathrm{mmol})$ and $\mathrm{Et}_{3} \mathrm{~N}(0.055 \mathrm{~mL}, 0.40 \mathrm{mmol})$ in $\mathrm{MeCN}$ $(12 \mathrm{~mL}$ ) over $2.5 \mathrm{~h}$ by an addition funnel. The addition funnel was rinsed with $3.5 \mathrm{~mL}$ of MeCN. The mixture was refluxed for an additional $3.5 \mathrm{~h}$. After being cooled to room temperature, the mixture was concentrated in vacuo. The residue was purified by column chromatography on silica gel (EtOAc/hexane, 1:4) to give $18.2 \mathrm{mg}(93 \%)$ of $\mathbf{4 8}$ as a colorless oil: TLC, $\mathrm{R}_{f} 0.76$ (EtOAc/hexane, 1:1); $[\alpha]^{26}{ }_{\mathrm{D}}+182\left(c 0.875, \mathrm{CHCl}_{3}\right) ;{ }^{1} \mathrm{H}$ NMR $(300 \mathrm{MHz}) \delta 0.83-0.98(\mathrm{~m}, 2 \mathrm{H}), 1.04(\mathrm{~d}, J=6.2 \mathrm{~Hz}, 3 \mathrm{H}), 1.12(\mathrm{~s}$, 3H), 1.16-1.29 (m, 6H), 1.34-1.50 (m, 2H), $1.52(\mathrm{~s}, 3 \mathrm{H}), 1.57-1.60(\mathrm{~m}, 7 \mathrm{H}), 1.63(\mathrm{~s}, 3 \mathrm{H}), 1.96-2.24(\mathrm{~m}$, 2H), $2.34(\mathrm{~d}, J=10.2 \mathrm{~Hz}, 1 \mathrm{H}), 2.39-2.51(\mathrm{~m}, 1 \mathrm{H}), 2.92(\mathrm{~m}, 1 \mathrm{H}), 3.37(\mathrm{~s}, 3 \mathrm{H}), 3.48(\mathrm{~d}, J=9.6 \mathrm{~Hz}, 1 \mathrm{H})$, 4.44, $4.58(2 \mathrm{~d}, J=6.4 \mathrm{~Hz}, 1 \mathrm{H} \times 2), 4.62-4.76(\mathrm{~m}, 1 \mathrm{H}), 5.09(\mathrm{~m}, 1 \mathrm{H}), 5.31(\mathrm{dd}, J=14.1,10.2 \mathrm{~Hz}, 1 \mathrm{H})$, $5.47(\mathrm{dd}, J=14.9,6.5 \mathrm{~Hz}, 1 \mathrm{H}), 5.73(\mathrm{~s}, 1 \mathrm{H}), 5.81-6.10(\mathrm{~m}, 2 \mathrm{H}) ;{ }^{13} \mathrm{C} \mathrm{NMR}(68 \mathrm{MHz}) \delta 11.2,15.7,16.7$, $18.7,19.2,22.6,25.3,26.4,26.8,33.3,34.8,36.7,38.1,40.9,45.6,48.5,54.3,55.5,71.2,79.6,88.1$, 
93.0, 120.5, 127.8, 130.9, 131.8, 132.8, $133.4 \times 2,135.5,174.2$; IR (neat) 3490, 2980, 2930, 2880, 1720, $1460 \mathrm{~cm}^{-1}$; HRMS (FAB) calcd for $\mathrm{C}_{31} \mathrm{H}_{48} \mathrm{O}_{5}\left(\mathrm{M}^{+}\right) \mathrm{m} / z$ 500.3502, found 500.3505 .

\section{(+)-Tubelactomicin B (2).}
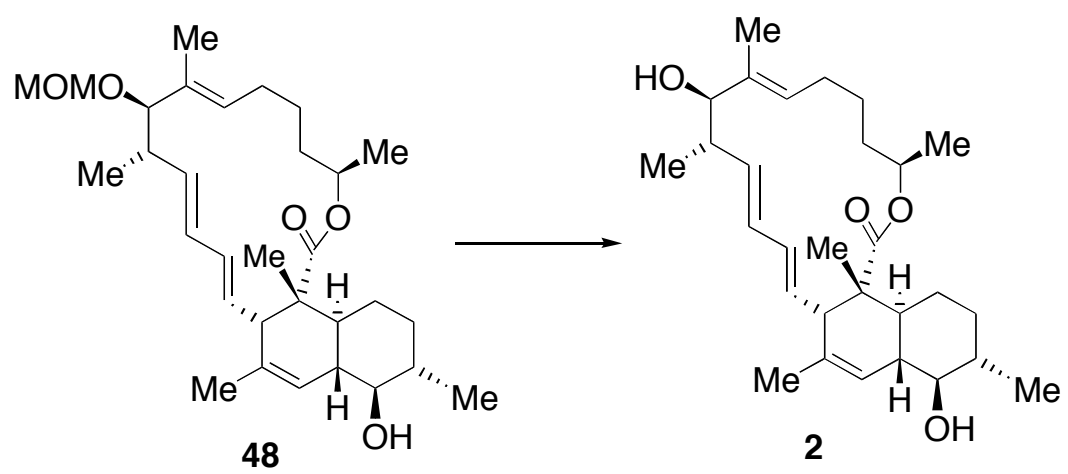

To a cooled $\left(0^{\circ} \mathrm{C}\right)$, stirred solution of $48(17.5 \mathrm{mg}, 34.9 \mu \mathrm{mol})$ in THF $(0.5 \mathrm{~mL})$ was added $6 \mathrm{M}$ aqueous $\mathrm{HCl}(0.5 \mathrm{~mL})$. The mixture was stirred for $11 \mathrm{~h}$ and then additional $6 \mathrm{M}$ aqueous $\mathrm{HCl}(0.25$ $\mathrm{mL})$ and THF $(0.5 \mathrm{~mL})$ were added. The mixture was stirred for additional $6 \mathrm{~h}$ and neutralized with saturated aqueous $\mathrm{NaHCO}_{3}(15 \mathrm{~mL})$ at $0{ }^{\circ} \mathrm{C}$. This was extracted with $\mathrm{CH}_{2} \mathrm{Cl}_{2}(8 \mathrm{~mL} \times 3)$. The combined organic layers were dried and concentrated in vacuo. The residue was purified by column chromatography on silica gel (EtOAc/hexane, 1:4) to give $13.3 \mathrm{mg}(83 \%)$ of 2 as a colorless solid: TLC, $\mathrm{R}_{f} 0.58\left(\mathrm{CHCl}_{3} / \mathrm{MeOH}, 10: 1\right), \mathrm{R}_{f} 0.62($ EtOAc/hexane, $1: 1) ;[\alpha]^{24.5}{ }_{\mathrm{D}}+101(c 0.60, \mathrm{MeOH}) ;{ }^{1} \mathrm{H}$ NMR $(300 \mathrm{MHz}) \delta$ 0.83-1.00 (m, 2H), $1.04(\mathrm{~d}, J=6.2 \mathrm{~Hz}, 3 \mathrm{H}), 1.12(\mathrm{~s}, 3 \mathrm{H}), 1.18(\mathrm{~d}, J=6.8 \mathrm{~Hz}, 3 \mathrm{H}), 1.21(\mathrm{~d}$, $J=6.2 \mathrm{~Hz}, 3 \mathrm{H}), 1.32-1.51(\mathrm{~m}, 2 \mathrm{H}), 1.60(\mathrm{~s}, 3 \mathrm{H}), 1.61-1.88(\mathrm{~m}, 7 \mathrm{H}), 1.63(\mathrm{~s}, 3 \mathrm{H}), 1.93-2.20(\mathrm{~m}, 2 \mathrm{H})$, 2.32-2.45 (m, 1H), $2.35(\mathrm{~d}, J=10.2 \mathrm{~Hz}, 1 \mathrm{H}), 2.92(\mathrm{~m}, 1 \mathrm{H}), 3.57(\mathrm{~d}, J=9.0 \mathrm{~Hz}, 1 \mathrm{H}), 4.68(\mathrm{~m}, 1 \mathrm{H}), 5.06$ (m, 1H), $5.31(\mathrm{dd}, J=14.0,10.2 \mathrm{~Hz}, 1 \mathrm{H}), 5.48(\mathrm{dd}, J=15.0,6.4 \mathrm{~Hz}, 1 \mathrm{H}), 5.73(\mathrm{~s}, 1 \mathrm{H}), 5.81-5.99(\mathrm{~m}$, $2 \mathrm{H}) ;{ }^{13} \mathrm{C}$ NMR $(68 \mathrm{MHz}) \delta 11.1,15.5,16.7,18.7,19.2,22.6,25.3,26.3,26.8,33.3,34.8,38.1,40.9$, 45.6, 48.5, 54.3, 71.3, 79.6, 85.3, 120.5, 127.6, 129.2, 130.8, 133.3, 133.4, 135.5, 136.2, 174.3; IR (KBr) 3420, 2980, 2930, 2880, 1720, 1705, $1460 \mathrm{~cm}^{-1}$; HRMS calcd for $\mathrm{C}_{29} \mathrm{H}_{44} \mathrm{O}_{4}\left(\mathrm{M}^{+}\right) \mathrm{m} / z$ 456.3240, found 426.3234. 


\section{hydroxy-7-methoxycarbonyl-6-methoxymethoxy-5-methyl)trideca-1,3,7-triethyl]-1,6-dimethyl-3-}

(methoxymethoxy)methyl-1,2,4a,5,6,7,8,8a-octahydronaphtalene-1-carboxylate (49).

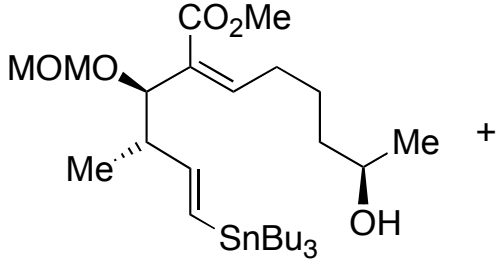

6

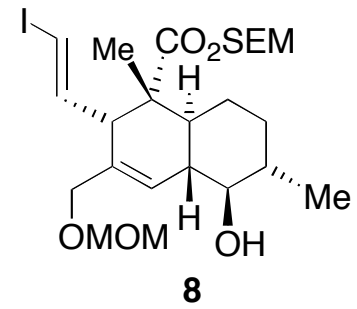

8

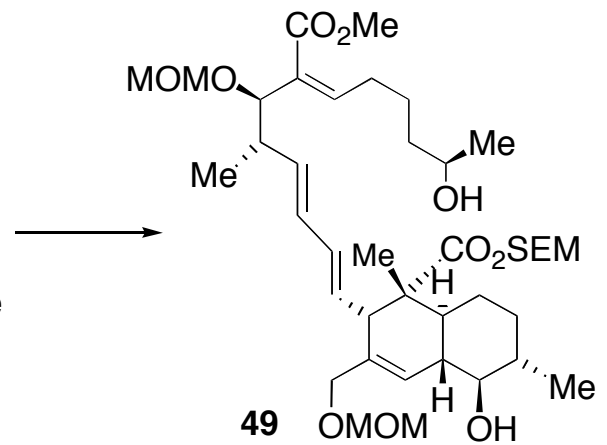

The following reaction was carried out under argon. To a stirred solution of $\mathbf{6}^{1 \mathrm{a}}(176 \mathrm{mg}, 0.30$ $\mathrm{mmol})$ and $8(145.4 \mathrm{mg}, 0.25 \mathrm{mmol})$ in degassed $\mathrm{DMF}(3 \mathrm{~mL})$ was added a solution of $\mathrm{Pd}_{2}(\mathrm{dba})_{3}(11.4$ $\mathrm{mg}, 12.4 \mu \mathrm{mol}), \mathrm{AsPPh}_{3}(30.6 \mathrm{mg}, 99.9 \mu \mathrm{mol})$ and $\mathrm{CuI}(19.0 \mathrm{mg}, 997 \mu \mathrm{mol})$ in degassed DMF (0.5 mL). The mixture was stirred for $20 \mathrm{~min}$ and then heated at $60{ }^{\circ} \mathrm{C}$ for $2 \mathrm{~h}$. After being cooled to $0{ }^{\circ} \mathrm{C}$, the mixture was diluted with saturated aqueous $\mathrm{NH}_{4} \mathrm{Cl}(4 \mathrm{~mL})$ and $\mathrm{H}_{2} \mathrm{O}(4 \mathrm{~mL})$ and extracted with $\mathrm{Et}_{2} \mathrm{O}(3$ $\mathrm{mL} \times 3$ ). The combined organic layers were dried and concentrated in with the aid of toluene. The residue was purified by column chromatography on silica gel (EtOAc/hexane, 1:2 to1:1) to give 49 (118 mg. 63\%) as a pale-yellow oil: TLC, $\mathrm{R}_{f} 0.25$ (EtOAc/hexane, $\left.2: 1\right) ;[\alpha]^{24.5}+124.8\left(c 1.84, \mathrm{CHCl}_{3}\right) ;{ }^{1} \mathrm{H}$ NMR $(300 \mathrm{MHz}) \delta 0.03(\mathrm{~s}, 9 \mathrm{H}), 0.95(\mathrm{t}, J=8.5 \mathrm{~Hz}, 2 \mathrm{H}), 1.04(\mathrm{t}, J=6.7 \mathrm{~Hz}, 5 \mathrm{H}), 1.18(\mathrm{t}, J=3.0 \mathrm{~Hz}$, 7H), 1.37-1.55 (m, 5H), 1.65-1.90 (m, 6H), 2.31-2.57 (m, 3H), $2.69(\mathrm{~d}, J=9.6 \mathrm{~Hz}, 1 \mathrm{H}), 2.93(\mathrm{dd}, J=$ $9.39 .3 \mathrm{~Hz}, 1 \mathrm{H}), 3.36(\mathrm{~s}, 6 \mathrm{H}), 3.68(\mathrm{t}, J=8.1 \mathrm{~Hz}, 2 \mathrm{H}), 3.72(\mathrm{~s}, 3 \mathrm{H}), 3.74-3.82(\mathrm{~m}, 1 \mathrm{H}), 3.90(2 \mathrm{~d}, J=12$ $\mathrm{Hz}, 1 \mathrm{H} \times 2), 4.15(\mathrm{~d}, J=7.2 \mathrm{~Hz}, 1 \mathrm{H}), 4.52(2 \mathrm{~d}, J=6.7 \mathrm{~Hz}, 1 \mathrm{H} \times 2), 4.62(2 \mathrm{~d}, J=5.0 \mathrm{~Hz}, 1 \mathrm{H} \times 2), 5.16$ $(2 \mathrm{~d}, J=6.0 \mathrm{~Hz}, 1 \mathrm{H} \times 2), 5.35(\mathrm{dd}, J=14,9.5 \mathrm{~Hz}, 2 \mathrm{H}), 5.49(\mathrm{dd}, J=8.0,14 \mathrm{~Hz}, 2 \mathrm{H}), 5.87-5.97(\mathrm{~m}, 2 \mathrm{H})$, $6.01(\mathrm{t}, J=7.7 \mathrm{~Hz}, 1 \mathrm{H}), 6.09(\mathrm{br} \mathrm{s}, 1 \mathrm{H}):{ }^{13} \mathrm{C} \mathrm{NMR}(68 \mathrm{MHz}) \delta-1.4 \times 3,15.6,16.4,18.0,18.6,23.5$, $25.5,27.0,29.2,33.2,38.0,38.4,41.0,41.1,45.4,49.3,49.7,51.3,55.3,55.8,67.5,67.8,69.4,79.0$, $80.1,89.4,94.3,95.3,124.8,129.9,130.9,131.1,132.6,133.6,135.8,143.2,167.4,174.7$; IR (neat) $3450,2950,1740,1450 \mathrm{~cm}^{-1}$. 


\section{1,2,4a,5,6,7,8,8a-octahydronaphtalene-1-carboxylate (50).}

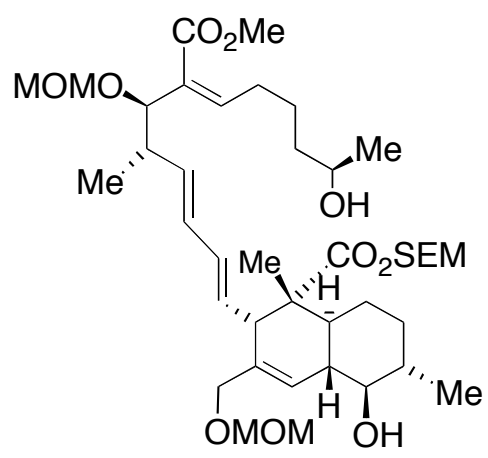

49

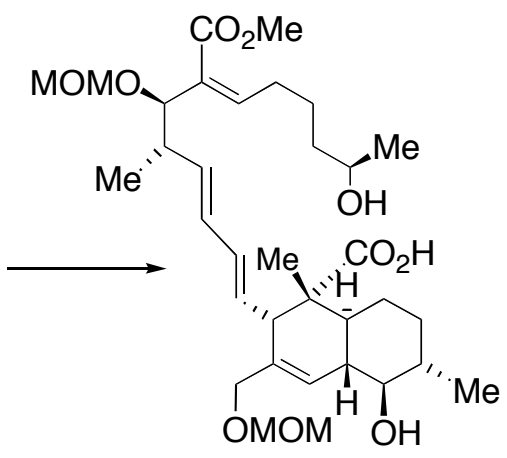

50

To a cooled $\left(0{ }^{\circ} \mathrm{C}\right)$, stirred solution of $49(118 \mathrm{mg}, 0.16 \mathrm{mmol})$ in THF $(2.4 \mathrm{~mL})$ was added HF.pyridine complex $(0.48 \mathrm{~mL})$. The mixture was stirred for $40 \mathrm{~h}$ and diluted with saturated aqueous $\mathrm{NaHCO}_{3}(6 \mathrm{~mL})$ and $\mathrm{H}_{2} \mathrm{O}(2 \mathrm{~mL})$. This was extracted with $\mathrm{Et}_{2} \mathrm{O}(3 \mathrm{~mL} \times 3)$. The combined organic layers were dried and concentrated with the aid of toluene. The residue was purified by column chromatography on silica gel (EtOAc/hexane, 1:1 to 3:1) to give $\mathbf{5 0}(99.6 \mathrm{mg}, 99 \%)$ as a colorless oil: TLC, $\mathrm{R}_{f} 0.43\left(\right.$ EtOAc/hexane, 4:1); $\left.[\alpha]_{\mathrm{D}}^{24.5}+106.9(c) 3.16, \mathrm{CHCl}_{3}\right) ;{ }^{1} \mathrm{H} \mathrm{NMR}(300 \mathrm{MHz}) \delta 1.02(\mathrm{~d}, J=$ $6.0 \mathrm{~Hz}, 3 \mathrm{H}), 1.04(\mathrm{~d}, J=6.0 \mathrm{~Hz}, 3 \mathrm{H}), 1.21(\mathrm{~m}, 1 \mathrm{H}), 1.17(\mathrm{~s}, 3 \mathrm{H}), 1.20(\mathrm{~d}, J=6.0 \mathrm{~Hz}, 3 \mathrm{H}), 1.39-1.54(\mathrm{~m}$, 5H), 1.68-1.82 (m, 4H), $2.23(\mathrm{~m}, 1 \mathrm{H}), 2.49-2.65(\mathrm{~m}, 2 \mathrm{H}), 2.71(\mathrm{~d}, J=9.6 \mathrm{~Hz}, 1 \mathrm{H}), 2.92(\mathrm{t}, J=9.0 \mathrm{~Hz}$, $1 \mathrm{H}), 3.35(\mathrm{~s}, 3 \mathrm{H}), 3.37(\mathrm{~s}, 3 \mathrm{H}), 3.72(\mathrm{~s}, 3 \mathrm{H}), 3.90(\mathrm{~m}, 1 \mathrm{H}), 3.90(2 \mathrm{~d}, J=12 \mathrm{~Hz}, 1 \mathrm{H} \times 2), 4.23(\mathrm{~d}, J=7.5$ $\mathrm{Hz}, 1 \mathrm{H}), 4.52(2 \mathrm{~d}, J=6.0 \mathrm{~Hz}, 1 \mathrm{H} \times 2), 4.62(2 \mathrm{~d}, J=6.0 \mathrm{~Hz}, 1 \mathrm{H} \times 2), 5.39(\mathrm{dd}, J=9.5,14 \mathrm{~Hz}, 1 \mathrm{H}), 5.59$ $(\mathrm{dd}, J=6.6,15 \mathrm{~Hz}, 1 \mathrm{H}), 5.87-5.98(\mathrm{~m}, 2 \mathrm{H}), 6.04(\mathrm{t}, J=7.7 \mathrm{~Hz}, 1 \mathrm{H}), 6.09(\mathrm{~s}, 1 \mathrm{H}) ;{ }^{13} \mathrm{C} \mathrm{NMR}(68 \mathrm{MHz}) \delta$ $15.7,16.5,18.7,23.2,25.1,26.9,29.1,33.3,37.9,38.4,40.9,41.0,45.5,48.7,49.4,51.4,55.3,55.8$, $67.9,69.5,79.1,79.9,94.3,95.3,124.8,130.2,130.7,131.1,132.4,133.8,134.3,143.7,167.6,177.7$; IR (neat) 2900, 2620, 1720, 1700, 1650, $1450 \mathrm{~cm}^{-1}$. 


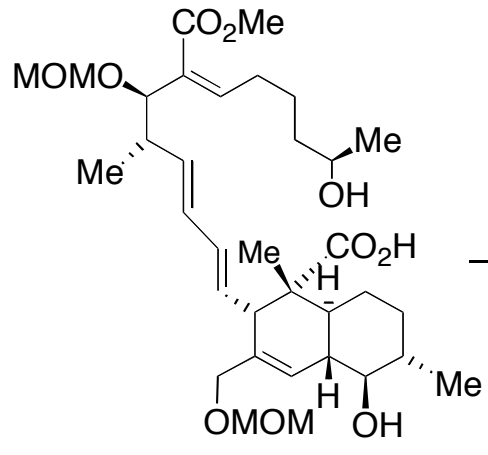

50

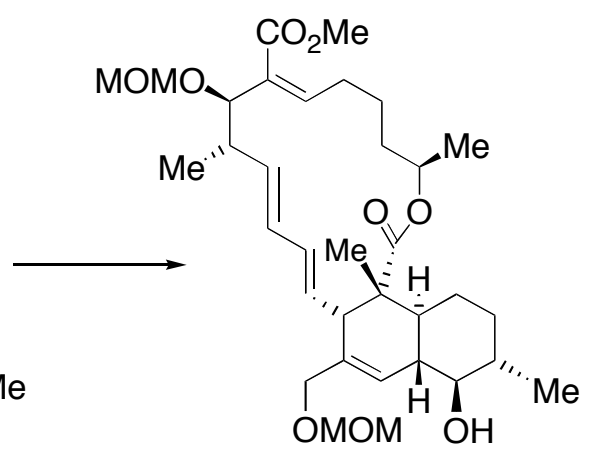

51

A solution of $\mathbf{5 0}(98.4 \mathrm{mg}, 0.16 \mathrm{mmol})$ in $\mathrm{MeCN}(30 \mathrm{~mL})$ was added to a refluxing solution of 2chloro-1-methylpyridinium iodide $(220 \mathrm{mg}, 0.86 \mathrm{mmol})$ and $\mathrm{Et}_{3} \mathrm{~N}(0.2 \mathrm{~mL}, 1.43 \mathrm{mmol})$ in $\mathrm{MeCN}(100$ $\mathrm{mL}$ ) over $1 \mathrm{~h}$ by an addition funnel. The addition funnel was rinsed with $3 \mathrm{~mL}$ of MeCN. The mixture was refluxed for an additional $5 \mathrm{~h}$. After being cooled to room temperature, the mixture was concentrated in vacuo. The residue was purified by column chromatography on silica gel (EtOAc/hexane, 1:2) to give $\mathbf{5 1}(58.0 \mathrm{mg}, 60 \%)$ as a colorless oil: TLC, $\mathrm{R}_{f} 0.75$ (EtOAc/hexane, 3:1); $[\alpha]_{\mathrm{D}}^{24.5}+108.6\left(c\right.$ 2.90, $\left.\mathrm{CHCl}_{3}\right) ;{ }^{1} \mathrm{H}$ NMR $(270 \mathrm{MHz}) \delta 1.01-1.21(\mathrm{~m}, 2 \mathrm{H}), 1.05(\mathrm{~d}, J=6.6 \mathrm{~Hz}, 3 \mathrm{H}), 1.12$ $(\mathrm{d}, J=5.8 \mathrm{~Hz}, 3 \mathrm{H}), 1.14(\mathrm{~s}, 3 \mathrm{H}), 1.20(\mathrm{~d}, J=6.2 \mathrm{~Hz}, 3 \mathrm{H}), 1.25-1.70(\mathrm{~m}, 4 \mathrm{H}), 1.74-1.92(\mathrm{~m}, 3 \mathrm{H}), 2.33$ (m, 1H), $2.54(\mathrm{ddd}, J=4.4,4.4,11 \mathrm{~Hz}, 1 \mathrm{H}), 2.67$ (d, $J=9.9 \mathrm{~Hz}, 1 \mathrm{H}), 2.84-3.09(\mathrm{~m}, 2 \mathrm{H}), 3.36(\mathrm{~s}, 6 \mathrm{H})$, $3.77(\mathrm{~s}, 3 \mathrm{H}), 3.90(\mathrm{~m}, 1 \mathrm{H}), 3.90(2 \mathrm{~d}, J=12 \mathrm{~Hz}, 1 \mathrm{H} \times 2), 3.90(2 \mathrm{~d}, J=6.9 \mathrm{~Hz}, 1 \mathrm{H} \times 2), 4.58(2 \mathrm{~d}, J=6.6$ $\mathrm{Hz}, 1 \mathrm{H} \times 2), 4.68-4.74(\mathrm{~m}, 1 \mathrm{H}), 5.30(\mathrm{dd}, J=3.3,10 \mathrm{~Hz}, 1 \mathrm{H}), 5.66-5.71(\mathrm{~m}, 2 \mathrm{H}), 5.94(\mathrm{dd}, J=14,7.0$ $\mathrm{Hz}, 2 \mathrm{H}), 6.60$ (br s, $1 \mathrm{H}) ;{ }^{13} \mathrm{C}$ NMR $(68 \mathrm{MHz}) \delta 15.5,16.6,18.6,19.1,25.1,26.9,28.6,33.2,34.8,38.2$, $38.4,41.0,45.4,48.2,49.8,51.3,55.2,55.6,69.4,71.0,79.1,84.6,93.9,95.3,124.5,128.2,130.2$, 131.0, 133.8, 133.8, 135.8, 145.8, 167.3, 174.1; IR (neat) 3550, 2940, 1700, $1450 \mathrm{~cm}^{-1}$; HRMS calcd for $\mathrm{C}_{34} \mathrm{H}_{52} \mathrm{O}_{9}\left(\mathrm{M}^{+}\right) m / z$ 604.3611, found 604.3612. 


\section{6,13,16,20-tetramethyl-24-hydroxymethyl-14-oxatricyclo[14.8.0.0 $\left.{ }^{17,22}\right]$ tetracosa-2,4,8,23-tetraene-}

\section{5-one (52).}

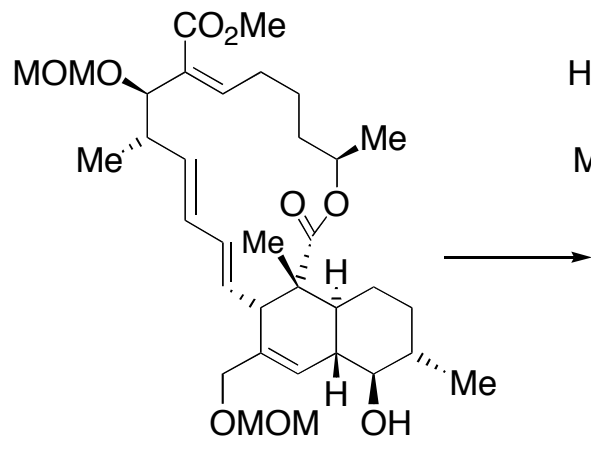

51

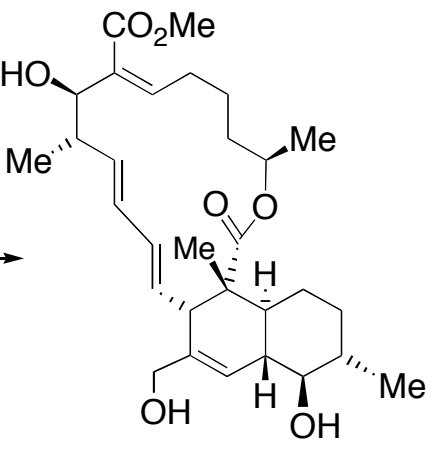

52

To a cooled $\left(0{ }^{\circ} \mathrm{C}\right)$, stirred solution of $\mathbf{5 1}(58.0 \mathrm{mg}, 95.9 \mu \mathrm{mol})$ in THF $(2 \mathrm{~mL})$ was added $6 \mathrm{M}$ aqueous $\mathrm{HCl}(2 \mathrm{~mL})$. The mixture was stirred for $25 \mathrm{~h}$ and neutralized with saturated aueous $\mathrm{NaHCO}_{3}$ (4 mL) at $0{ }^{\circ} \mathrm{C}$. This was extracted with $\mathrm{CH}_{2} \mathrm{Cl}_{2}(4 \mathrm{~mL} \times 3)$. The combined organic layers were dried and concentrated in vacuo. The residue was purified by column chromatography on silica gel (EtOAc/hexane, 2:1) to give $\mathbf{5 2}(47.7 \mathrm{mg}, 96 \%)$ as a colorless oil: TLC, $\mathrm{R}_{f} 0.32$ (EtOAc/hexane, 3:1); $[\alpha]_{\mathrm{D}}^{24.5}+86.1\left(c\right.$ 2.39, $\left.\mathrm{CHCl}_{3}\right) ;{ }^{1} \mathrm{H}$ NMR $(270 \mathrm{MHz}) \delta 0.91-1.07(\mathrm{~m}, 2 \mathrm{H}), 1.02(\mathrm{~d}, J=6.2 \mathrm{~Hz}, 3 \mathrm{H}), 1.11$ $(\mathrm{s}, 3 \mathrm{H}), 1.18(\mathrm{~d}, J=6.2 \mathrm{~Hz}, 3 \mathrm{H}), 1.20(\mathrm{~d}, J=6.0 \mathrm{~Hz}, 3 \mathrm{H}), 1.29-1.85(\mathrm{~m}, 9 \mathrm{H}), 2.44(\mathrm{~m}, 1 \mathrm{H}), 2.53(\mathrm{~d}, J=$ $10 \mathrm{~Hz}, 1 \mathrm{H}), 2.59-2.61(\mathrm{~m}, 2 \mathrm{H}), 2.91(\mathrm{t}, J=4.5 \mathrm{~Hz}, 1 \mathrm{H}), 3.62(\mathrm{~m}, 1 \mathrm{H}), 3.80(\mathrm{~s}, 3 \mathrm{H}), 3.92(2 \mathrm{~d}, J=13 \mathrm{~Hz}$, $1 \mathrm{H} \times 2), 4.71(\mathrm{~m}, 1 \mathrm{H}), 5.31(\mathrm{dd}, J=10,13 \mathrm{~Hz}, 1 \mathrm{H}), 5.52(\mathrm{dd}, J=6.4,14 \mathrm{~Hz}, 1 \mathrm{H}), 5.80-5.95(\mathrm{~m}, 3 \mathrm{H})$, 6.05 (s, 1H); ${ }^{13} \mathrm{C}$ NMR (68 MHz) $\delta 15.5,16.5,18.6,19.1,25.2,26.9,28.5,33.3,35.1,38.5,40.2,41.0$, $45.3,48.3,50.2,51.4,64.9,71.0,78.9,83.2,120.8,127.6,131.0,132.2,133.1,136.3,146.9,167.7$, 174.1; IR (neat) 3300, 2950, 1700, $1450 \mathrm{~cm}^{-1}$; HRMS calcd for $\mathrm{C}_{30} \mathrm{H}_{44} \mathrm{O}_{7}\left(\mathrm{M}^{+}\right) \mathrm{m} / z$ 516.3087, found 516.3091 . 
(+)-Tubelactomicin D (3).

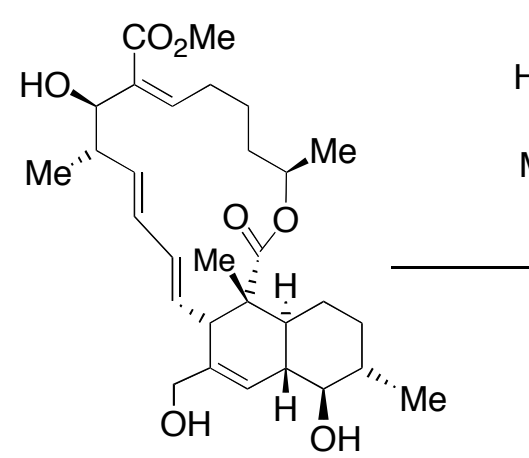

52

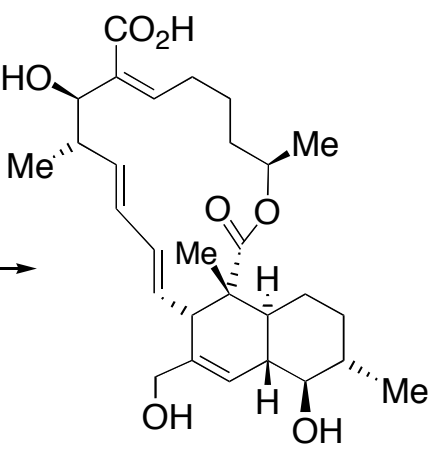

3

To a stirred solution of $\mathbf{5 2}(40.1 \mathrm{mg}, 77.6 \mu \mathrm{mol})$ in $\mathrm{MeOH}(3 \mathrm{~mL})$ was added $1 \mathrm{M}$ aqueous $\mathrm{NaOH}$ $(1.5 \mathrm{~mL})$. The mixture was heated at $50{ }^{\circ} \mathrm{C}$ with stirring for $6.5 \mathrm{~h}$. After being cooled to $0{ }^{\circ} \mathrm{C}$, the mixture was acidified with $1 \mathrm{M}$ aqueous $\mathrm{HCl}$ to $\mathrm{pH} 2$. This was diluted with $\mathrm{H}_{2} \mathrm{O}(4 \mathrm{~mL})$ and extracted with $\mathrm{CH}_{2} \mathrm{Cl}_{2}(3 \mathrm{~mL} \times 3)$. The combined organic layers were dried and concentrated in vacuo. The residue was purified by column chromatography on silica gel (hexane/acetone, 2:1) to give $\mathbf{3}$ (36.0 $\mathrm{mg}$, 92\%) as a colorless oil: TLC, $\mathrm{R}_{f} 0.22\left(\mathrm{CHCl}_{3} / \mathrm{MeOH}, 10: 1\right.$ containing $\left.1 \% \mathrm{AcOH}\right) ;[\alpha]_{\mathrm{D}}^{24.5}+95.7(c 0.14$, $\left.\mathrm{CHCl}_{3}\right) ;{ }^{1} \mathrm{H}$ NMR $\left(300 \mathrm{MHz}\right.$, acetone- $\left.d_{6}\right) \delta 1.03(\mathrm{~d}, J=6.4 \mathrm{~Hz}, 3 \mathrm{H}), 1.12(\mathrm{~s}, 3 \mathrm{H}), 1.15(\mathrm{~d}, J=6.0 \mathrm{~Hz}$, $3 \mathrm{H}), 1.21(\mathrm{~d}, J=7.3 \mathrm{~Hz}, 3 \mathrm{H}), 1.27-1.33(\mathrm{~m}, 3 \mathrm{H}), 1.34-1.71(\mathrm{~m}, 3 \mathrm{H}), 1.60-1.78(\mathrm{~m}, 4 \mathrm{H}), 1.84-1.88(\mathrm{~m}$, $1 \mathrm{H}), 2.40-2.60(\mathrm{~m}, 2 \mathrm{H}), 2.72(\mathrm{~d}, J=10 \mathrm{~Hz}, 2 \mathrm{H}), 2.84(\mathrm{t}, J=9.0 \mathrm{~Hz}, 2 \mathrm{H}), 3.89-3.93(\mathrm{~m}, 5 \mathrm{H}), 4.71(\mathrm{~m}$, $1 \mathrm{H}), 5.28(\mathrm{dd}, J=10,14 \mathrm{~Hz}, 1 \mathrm{H}), 5.72(\mathrm{dd}, J=5.7,14 \mathrm{~Hz}, 1 \mathrm{H}), 5.81(\mathrm{dd}, J=4.6,8.3 \mathrm{~Hz}, 1 \mathrm{H}), 5.89$ $6.00(\mathrm{~m}, 2 \mathrm{H}), 6.09$ (br s, $1 \mathrm{H}) ;{ }^{13} \mathrm{C}$ NMR $\left(75 \mathrm{MHz}\right.$, acetone- $\left.d_{6}\right) \delta 15.9,17.1,19.4,19.5,26.1,27.8,29.0$, $34.4,35.9,39.6,41.2,41.9,46.5,48.9,50.5,65.1,71.4,79.4,81.8,122.9,128.7,131.7,134.3,134.7$, 137.0, 137.7, 144.7, 168.6, 174.4; IR (neat) 3600, 3500, 2980, 1740, 1680, 1650, $1460 \mathrm{~cm}^{-1}$; HRMS calcd for $\mathrm{C}_{29} \mathrm{H}_{42} \mathrm{O}_{7}\left(\mathrm{M}^{+}\right) \mathrm{m} / z$ 502.2930, found 502.2929.

2-[(Trimethylsilyl)ethoxy]methyl $(1 S, 2 R, 5 S, 6 R, 7 S, 10 R, 13 R)-5$-ethynyl-4,6-dimethyl-13-phenyl12,14-dioxatricyclo[8.4.0.0 $\left.{ }^{2,7}\right]$ tetradec-3-ene-6-carboxylate (54). 


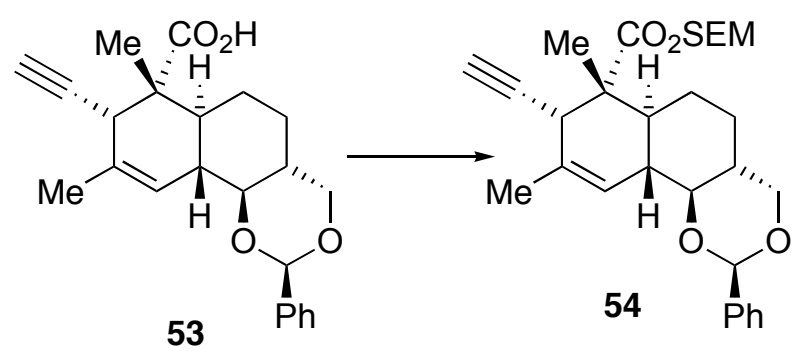

The following reaction was carried out under argon. To a cooled $\left(0{ }^{\circ} \mathrm{C}\right)$, stirred solution of $\mathbf{5 3}^{\text {lb }}(258$ $\mathrm{mg}, 0.705 \mathrm{mmol})$ in THF $(5 \mathrm{~mL})$ were added $\mathrm{Et}_{3} \mathrm{~N}(0.20 \mathrm{~mL}, 1.4 \mathrm{mmol})$ and SEMCl $(0.13 \mathrm{~mL}, 0.71$ mmol). The mixture was stirred $0{ }^{\circ} \mathrm{C}$ for $1.5 \mathrm{~h}$ and diluted with saturated aqueous $\mathrm{NH}_{4} \mathrm{Cl}(15 \mathrm{~mL})$. This was extracted with $\mathrm{CH}_{2} \mathrm{Cl}_{2}(8 \mathrm{~mL} \times 3)$. The combined organic layers were dried and concentrated in vacuo. The residue was purified by column chromatography on silica gel (EtOAc/hexane, 1:20) to give $323 \mathrm{mg}(92 \%)$ of $\mathbf{5 4}$ as a colorless oil: TLC, $\mathrm{R}_{f} 0.73$ (EtOAc/hexane, 1:2); $[\alpha]^{24.5}+9.3\left(c 2.07, \mathrm{CHCl}_{3}\right)$; ${ }^{1} \mathrm{H}$ NMR (300 MHz) $\delta 0.03(\mathrm{~s}, 9 \mathrm{H}), 0.98(\mathrm{t}, J=8.3 \mathrm{~Hz}, 2 \mathrm{H}), 1.13-1.22(\mathrm{~m}, 2 \mathrm{H}), 1.21(\mathrm{~s}, 3 \mathrm{H}), 1.60-1.67$ (m, 1H), 1.80-1.92 (m, 1H), $1.85(\mathrm{~s}, 3 \mathrm{H}), 1.98-2.10(\mathrm{~m}, 2 \mathrm{H}), 2.16-2.20(\mathrm{~m}, 1 \mathrm{H}), 2.17(\mathrm{~d}, J=2.4 \mathrm{~Hz}, 1 \mathrm{H})$, $2.85(\mathrm{~s}, 1 \mathrm{H}), 3.37(\mathrm{dd}, J=9.6,9.6 \mathrm{~Hz}, 1 \mathrm{H}), 3.62(\mathrm{dd}, J=11.0,11.0 \mathrm{~Hz}, 1 \mathrm{H}), 3.75(\mathrm{~m}, 2 \mathrm{H}), 4.12(\mathrm{dd}, J=$ 11.0, $4.4 \mathrm{~Hz}, 1 \mathrm{H}), 5.33,5.36(2 \mathrm{~d}, J=6.1 \mathrm{~Hz}, 1 \mathrm{H} \times 2), 5.56(\mathrm{~s}, 1 \mathrm{H}), 5.78(\mathrm{~s}, 1 \mathrm{H}), 7.31-7.41(\mathrm{~m}, 3 \mathrm{H})$, 7.51-7.53 (m, 2H); ${ }^{13} \mathrm{C}$ NMR (75 MHz) $\delta-1.4 \times 3,16.1,18.0,22.4,25.5,26.1,38.2,41.6,42.4,42.5$, $48.8,67.9,71.6,71.8,83.1,83.7,89.6,101.5,120.6,126.0 \times 2,128.2 \times 2,128.7,130.8,138.6,173.8 ;$ IR (neat) $3310,2955,2920,2890,2850,1750,1730,1715,1455 \mathrm{~cm}^{-1}$; HRMS calcd for $\mathrm{C}_{29} \mathrm{H}_{40} \mathrm{O}_{5} \mathrm{Si}\left(\mathrm{M}^{+}\right)$ $m / z 496.2645$, found 496.2632 .

\section{2-(Trimethylsilyl)ethoxymethyl $\quad(1 S, 2 R, 5 S, 6 R, 7 S, 10 R, 13 R)-5-[(E)$-2-iodoethenyl]-4,6-dimethyl-} 13-phenyl-12,14-dioxatricyclo[8.4.0.0 $\left.{ }^{2,7}\right]$ tetradec-3-ene-6-carboxylate (55).

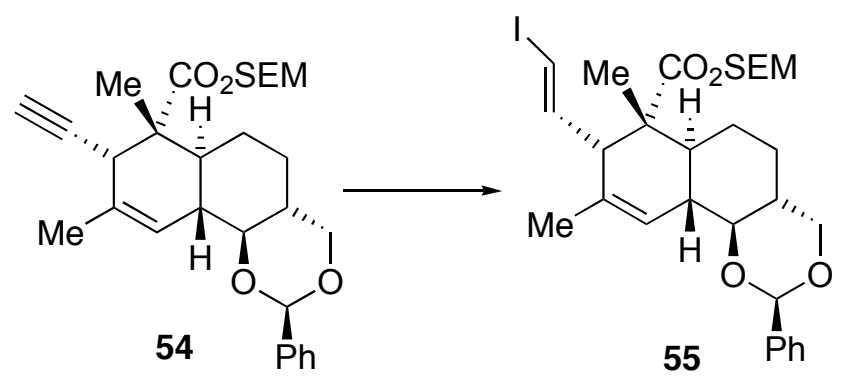


The following reaction was carried out under argon. To a stirred solution of $\mathbf{5 4}(132 \mathrm{mg}, 0.27 \mathrm{mmol})$ in THF $(2 \mathrm{~mL})$ were added $\mathrm{Pd}\left(\mathrm{PPh}_{3}\right)_{2} \mathrm{Cl}_{2}(18.7 \mathrm{mg}, 26.6 \mu \mathrm{mol})$ and $\mathrm{Bu}_{3} \mathrm{SnH}(0.14 \mathrm{~mL}, 0.53 \mathrm{mmol})$. The reaction mixture was stirred for $20 \mathrm{~min}$ and concentrated in vacuo. The residue was purified by column chromatography on silica gel (EtOAc/hexane, 1:30 doped with $\left.\mathrm{Et}_{3} \mathrm{~N}\right)$ to give $189 \mathrm{mg}$ of vinylstannanes as a colorless oil. This material was dissolved in $\mathrm{CH}_{2} \mathrm{Cl}_{2}(4 \mathrm{~mL})$ and stirred at $0{ }^{\circ} \mathrm{C}$. To the solution was added $\mathrm{I}_{2}(61.0 \mathrm{mg}, 0.24 \mathrm{mmol})$. The mixture was stirred for $2 \mathrm{~h}$ and diluted with $20 \%$ aqueous $\mathrm{Na}_{2} \mathrm{~S}_{2} \mathrm{O}_{3}$ $(12 \mathrm{~mL})$ at $0{ }^{\circ} \mathrm{C}$. This was stirred for $5 \mathrm{~min}$ and extracted with $\mathrm{CH}_{2} \mathrm{Cl}_{2}(6 \mathrm{~mL} \times 3)$. The combined organic layers were dried and concentrated in vacuo. The residue was purified by column chromatography on silica gel (EtOAc/hexane, 1:30 doped with $\left.\mathrm{Et}_{3} \mathrm{~N}\right)$ to give $121 \mathrm{mg}(73 \%, 2$ steps) of 55 as a colorless oil; TLC, $\mathrm{R}_{f} 0.47$ (EtOAc/hexane, 1:5); $[\alpha]^{21.5}{ }_{\mathrm{D}}+62.3\left(c\right.$ 2.16, $\left.\mathrm{CHCl}_{3}\right) ;{ }^{1} \mathrm{H}$ NMR $(300$ MHz) $\delta 0.04(\mathrm{~s}, 9 \mathrm{H}), 0.99(\mathrm{t}, J=8.3 \mathrm{~Hz}, 2 \mathrm{H}), 1.12-1.22(\mathrm{~m}, 2 \mathrm{H}), 1.19(\mathrm{~s}, 3 \mathrm{H}), 1.60-1.68(\mathrm{~m}, 1 \mathrm{H}), 1.64$ (s, 3H), 1.80-1.94 (m, 3H), $2.06(\mathrm{dd}, J=10.0,10.0 \mathrm{~Hz}, 1 \mathrm{H}), 2.44(\mathrm{~d}, J=10.0 \mathrm{~Hz}, 1 \mathrm{H}), 3.31(\mathrm{dd}, J=10.0$, $10.0 \mathrm{~Hz}, 1 \mathrm{H}), 3.63(\mathrm{dd}, J=11.0,11.0 \mathrm{~Hz}, 1 \mathrm{H}), 3.73(\mathrm{t}, J=8.3 \mathrm{~Hz}, 2 \mathrm{H}), 4.13(\mathrm{dd}, J=11.0,4.1 \mathrm{~Hz}, 1 \mathrm{H})$, $5.18,5.35(2 \mathrm{~d}, J=6.1 \mathrm{~Hz}, 1 \mathrm{H} \times 2), 5.57(\mathrm{~s}, 1 \mathrm{H}), 5.81(\mathrm{~s}, 1 \mathrm{H}), 6.06(\mathrm{~d}, J=14.4 \mathrm{~Hz}, 1 \mathrm{H}), 6.36(\mathrm{dd}, J$ $=14.4,10.0 \mathrm{~Hz}, 1 \mathrm{H}), 7.32-7.42(\mathrm{~m}, 3 \mathrm{H}), 7.52-7.54(\mathrm{~m}, 2 \mathrm{H}) ;{ }^{13} \mathrm{C} \mathrm{NMR}(68 \mathrm{MHz}) \delta-1.4 \times 3,16.4,18.1$, $22.3,25.5,26.2,38.0,41.5,42.4,49.1,57.6,68.2,71.8,76.5,76.7,84.0,89.9,101.5,121.1,126.0 \times 2$, $128.2 \times 2,128.7,131.0,138.5,145.4,174.3 ;$ IR (neat) 3040, 2955, 2930, 2890, 2855, 1730, 1715, 1595, $1460 \mathrm{~cm}^{-1}$; HRMS calcd for $\mathrm{C}_{29} \mathrm{H}_{41} \mathrm{O}_{5} \mathrm{SiI}\left(\mathrm{M}^{+}\right) \mathrm{m} / z$ 624.1768, found 624.1773. 


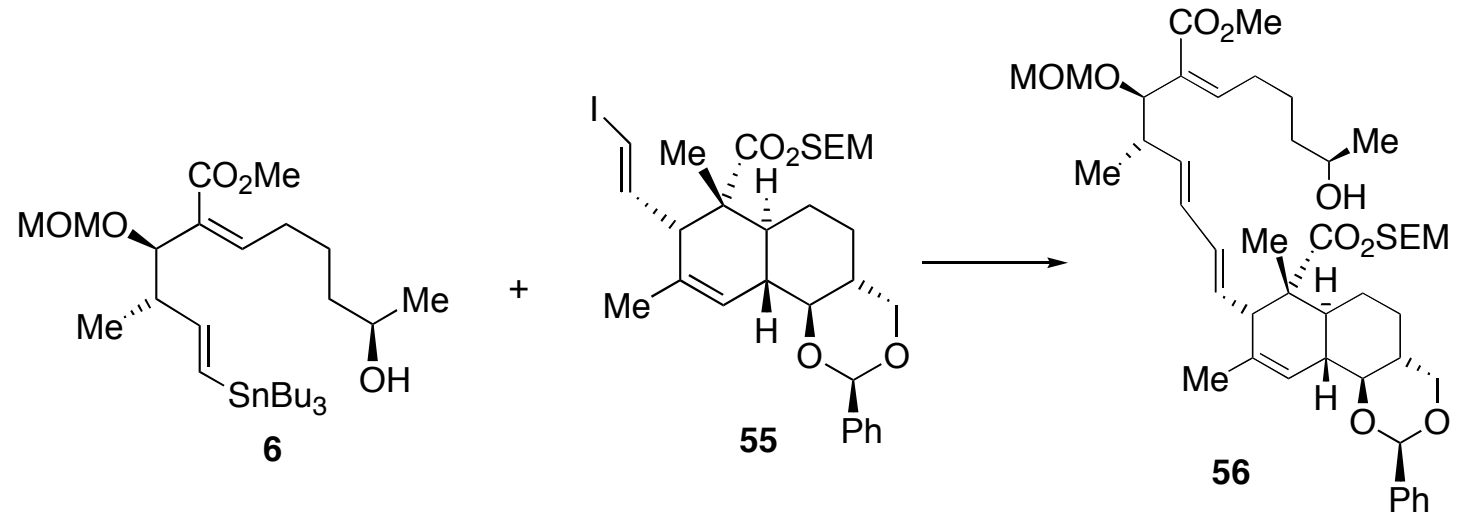

The following reaction was carried out under argon. To a stirred solution of $\mathbf{6}^{3 \mathrm{~b}}(103 \mathrm{mg}, 0.175$ $\mathrm{mmol})$ and $55(92.3 \mathrm{mg}, 0.15 \mathrm{mmol})$ in degassed DMF $(2 \mathrm{~mL})$ was added a solution of $\operatorname{Pd}_{2}(\mathrm{dba})_{3}(6.8$ $\mathrm{mg}, 7.4 \mu \mathrm{mol}), \mathrm{AsPh}_{3}(18.1 \mathrm{mg}, 59.1 \mu \mathrm{mol})$ and $\mathrm{CuI}(5.6 \mathrm{mg}, 30 \mu \mathrm{mol})$ in degassed DMF $(0.5 \mathrm{~mL})$. The mixture was stirred for $30 \mathrm{~min}$ and heated to $60^{\circ} \mathrm{C}$ for $1 \mathrm{~h}$. After being cooled to room temperature, the mixture was diluted with saturated aqueous $\mathrm{NH}_{4} \mathrm{Cl}(15 \mathrm{~mL})$ and extracted with $\mathrm{Et}_{2} \mathrm{O}(8 \mathrm{~mL} \times 3)$. The combined organic layers were dried and concentrated with the aid of toluene. The residue was purified by column chromatography on silica gel (EtOAc/hexane, 1:4 to 1:2) to provide $107 \mathrm{mg}(91 \%)$ of 56 as a colorless oil: TLC, $\mathrm{R}_{f} 0.47($ EtOAc/hexane, $1: 1) ;[\alpha]^{23}{ }_{\mathrm{D}}+67.0\left(c 0.99, \mathrm{CHCl}_{3}\right) ;{ }^{1} \mathrm{H}$ NMR $(300$ MHz) $\delta 0.04(\mathrm{~s}, 9 \mathrm{H}), 0.96(\mathrm{t}, J=8.3 \mathrm{~Hz}, 2 \mathrm{H}), 1.03(\mathrm{~d}, J=6.6 \mathrm{~Hz}, 3 \mathrm{H}), 1.17-1.22(\mathrm{~m}, 2 \mathrm{H}), 1.17(\mathrm{~d}, J=$ $6.3 \mathrm{~Hz}, 3 \mathrm{H}), 1.20(\mathrm{~s}, 3 \mathrm{H}), 1.42-1.56(\mathrm{~m}, 4 \mathrm{H}), 1.62(\mathrm{~s}, 3 \mathrm{H}), 1.62-1.90(\mathrm{~m}, 4 \mathrm{H}), 2.04-2.10(\mathrm{~m}, 1 \mathrm{H}), 2.30-$ $2.44(\mathrm{~m}, 1 \mathrm{H}), 2.39(\mathrm{~d}, J=9.8 \mathrm{~Hz}, 1 \mathrm{H}), 2.44-2.54(\mathrm{~m}, 2 \mathrm{H}), 3.31(\mathrm{dd}, J=9.7,9.7 \mathrm{~Hz}, 1 \mathrm{H}), 3.36(\mathrm{~s}, 3 \mathrm{H})$, $3.62(\mathrm{dd}, J=11.0,11.0 \mathrm{~Hz}, 1 \mathrm{H}), 3.69(\mathrm{t}, J=8.3 \mathrm{~Hz}, 2 \mathrm{H}), 3.72(\mathrm{~s}, 3 \mathrm{H}), 3.74-3.82(\mathrm{~m}, 1 \mathrm{H}), 4.13(\mathrm{dd}, J=$ 11.0, $4.0 \mathrm{~Hz}, 1 \mathrm{H}), 4.21(\mathrm{~d}, J=6.6 \mathrm{~Hz}, 1 \mathrm{H}), 4.49,4.60(2 \mathrm{~d}, J=6.6 \mathrm{~Hz}, 1 \mathrm{H} \times 2), 5.15,5.19(2 \mathrm{~d}, J=6.0$ $\mathrm{Hz}, 1 \mathrm{H} \times 2), 5.38(\mathrm{dd}, J=14.1,9.8 \mathrm{~Hz}, 1 \mathrm{H}), 5.53(\mathrm{dd}, J=14.4,7.3 \mathrm{~Hz}, 1 \mathrm{H}), 5.58(\mathrm{~s}, 1 \mathrm{H}), 5.75(\mathrm{~s}, 1 \mathrm{H})$, 5.88-6.01 (m, 2H), $6.05(\mathrm{t}, J=7.6 \mathrm{~Hz}, 1 \mathrm{H}), 7.32-7.40(\mathrm{~m}, 3 \mathrm{H}), 7.52-7.55(\mathrm{~m}, 2 \mathrm{H}) ;{ }^{13} \mathrm{C} \mathrm{NMR}(75 \mathrm{MHz}) \delta$ $-1.4 \times 3,15.3,16.5,18.0,22.4,23.5,25.4,25.6,26.2,29.1,37.9,38.7,41.2,41.5,42.4,49.6,51.3,54.4$, $55.8,67.5,67.8,71.9,79.8,84.3,89.5,94.4,101.5,119.8,126.0 \times 2,128.1 \times 2,128.6,129.9,131.1$, $131.5,132.4,132.9,135.6,138.6,143.1,167.4,174.7$; IR (neat) 3520, 2955, 2930, 2890, 2850, 1730, $1715,1475 \mathrm{~cm}^{-1}$; HRMS calcd for $\mathrm{C}_{45} \mathrm{H}_{69} \mathrm{O}_{10} \mathrm{Si}\left(\mathrm{M}^{+}+\mathrm{H}\right) \mathrm{m} / z .797 .4661$, found 797.4673 . 


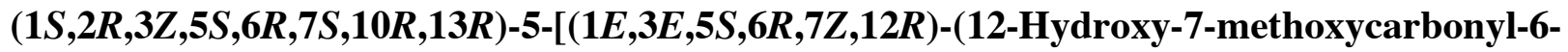

methoxymethoxy-5-methyl)trideca-1,3,7-trienyl]-4,6-dimethyl-13-phenyl-12,14-

dioxatricyclo $\left[8.4 .0 .0^{2,7}\right]$ tetradec-3-ene-6-carboxylate (57).

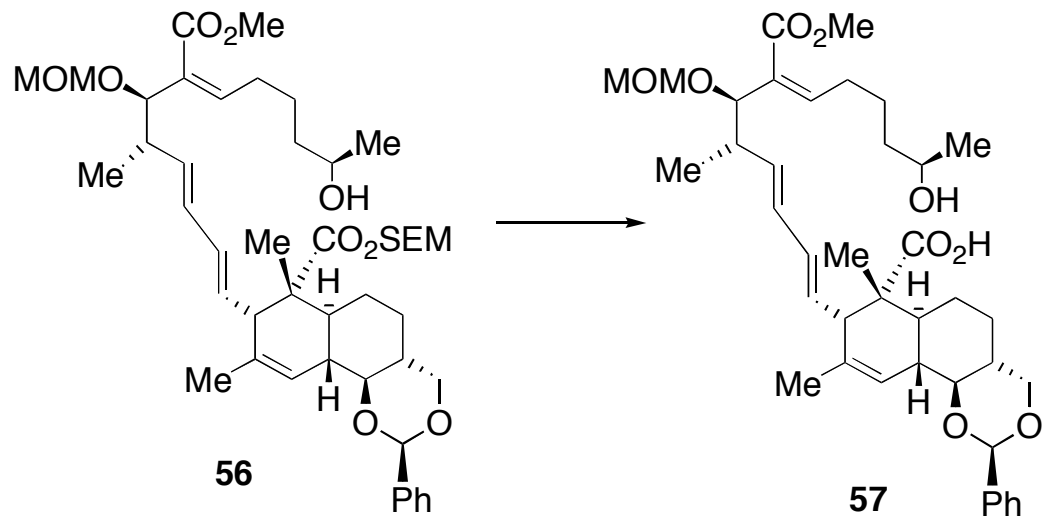

To a cooled $\left(0^{\circ} \mathrm{C}\right)$, stirred solution of $\mathbf{5 6}(38.8 \mathrm{mg}, 48.7 \mu \mathrm{mol})$ in THF/pyridine $(14: 1,1 \mathrm{~mL})$ was added HF-pyridine complex $(0.2 \mathrm{~mL})$. The mixture was stirred for $20 \mathrm{~h}$ and additional HF-pyridine complex $(0.025 \mathrm{~mL})$ was added at $0{ }^{\circ} \mathrm{C}$. The mixture was stirred for 3 days and quenched with saturated aqueous $\mathrm{NaHCO}_{3}(2 \mathrm{~mL})$ at $0{ }^{\circ} \mathrm{C}$. This was diluted with saturated aqueous $\mathrm{NaHCO}_{3}(8 \mathrm{~mL})$ and extracted with $\mathrm{CH}_{2} \mathrm{Cl}_{2}(5 \mathrm{~mL} \times 3)$. The combined organic layers were dried and concentrated with the aid of toluene. The residue was purified by column chromatography on silica gel (EtOAc/hexane, 1:2) to give $28.1 \mathrm{mg}(86 \%)$ of $\mathbf{5 7}$ as a colorless oil: TLC, $\mathrm{R}_{f} 0.42($ EtOAc/hexane, $2: 1) ;[\alpha]_{\mathrm{D}}^{26}+42.6(c 1.25$, $\left.\mathrm{CHCl}_{3}\right) ;{ }^{1} \mathrm{H}$ NMR $(300 \mathrm{MHz}) \delta 1.04(\mathrm{~d}, J=6.9 \mathrm{~Hz}, 3 \mathrm{H}), 1.13-1.24(\mathrm{~m}, 2 \mathrm{H}), 1.19(\mathrm{~s}, 3 \mathrm{H}), 1.21(\mathrm{~d}, J=6.3$ $\mathrm{Hz}, 3 \mathrm{H}), 1.47-1.56(\mathrm{~m}, 4 \mathrm{H}), 1.60-1.98$ (m, 4H), 1.63 (s, 3H), 2.00-2.09 (m, 1H), 2.20-2.34 (m, 1H), 2.42 $(\mathrm{d}, J=9.6 \mathrm{~Hz}, 1 \mathrm{H}), 2.50-2.65(\mathrm{~m}, 2 \mathrm{H}), 3.30(\mathrm{dd}, J=9.6,9.6 \mathrm{~Hz}, 1 \mathrm{H}), 3.37(\mathrm{~s}, 3 \mathrm{H}), 3.62(\mathrm{dd}, J=11.0$, $11.0 \mathrm{~Hz}, 1 \mathrm{H}), 3.73(\mathrm{~s}, 3 \mathrm{H}), 3.86-3.97(\mathrm{~m}, 1 \mathrm{H}), 4.12(\mathrm{dd}, J=11.0,4.1 \mathrm{~Hz}, 1 \mathrm{H}), 4.26(\mathrm{~d}, J=7.2 \mathrm{~Hz}, 1 \mathrm{H})$, $4.52,4.63(2 \mathrm{~d}, J=6.6 \mathrm{~Hz}, 1 \mathrm{H} \times 2), 5.43(\mathrm{dd}, J=13.8,9.6 \mathrm{~Hz}, 1 \mathrm{H}), 5.57(\mathrm{~s}, 1 \mathrm{H}), 5.65(\mathrm{dd}, J=14.7,6.6$ $\mathrm{Hz}, 1 \mathrm{H}), 5.74(\mathrm{~s}, 1 \mathrm{H}), 5.90-6.00(\mathrm{~m}, 2 \mathrm{H}), 6.06(\mathrm{t}, J=7.6 \mathrm{~Hz}, 1 \mathrm{H}), 7.34-7.40(\mathrm{~m}, 3 \mathrm{H})$, 7.52-7.54 (m, 2H); ${ }^{13} \mathrm{C}$ NMR $(75 \mathrm{MHz}) \delta 15.6,16.7,22.5,23.2,25.1,25.7,26.1,29.0,37.8,38.4,40.8,41.6,42.6,49.0$, $51.4,54.0,55.8,68.0,71.9,79.8,84.4,94.3,101.5,119.8,126.0 \times 2,128.2 \times 2,128.6,130.2,130.7$, 131.6, 132.2, 133.1, 134.0, 138.7, 143.7, 167.6, 177.6; IR (neat) 3460, 3020, 2960, 2930, 2855, 1720, $1715,1700,1455 \mathrm{~cm}^{-1}$; HRMS calcd for $\mathrm{C}_{39} \mathrm{H}_{54} \mathrm{O}_{9}\left(\mathrm{M}^{+}\right) \mathrm{m} / z$ 666.3768, found 666.3776. 


\section{6,13,16,28-tetramethyl-23-phenyl-14,22,24-trioxatetracyclo[14.12.0.0 $\left.{ }^{17,26} 0^{20,25}\right]$ octacosa-2,4,8,27-}

tetraene-15-one (58).

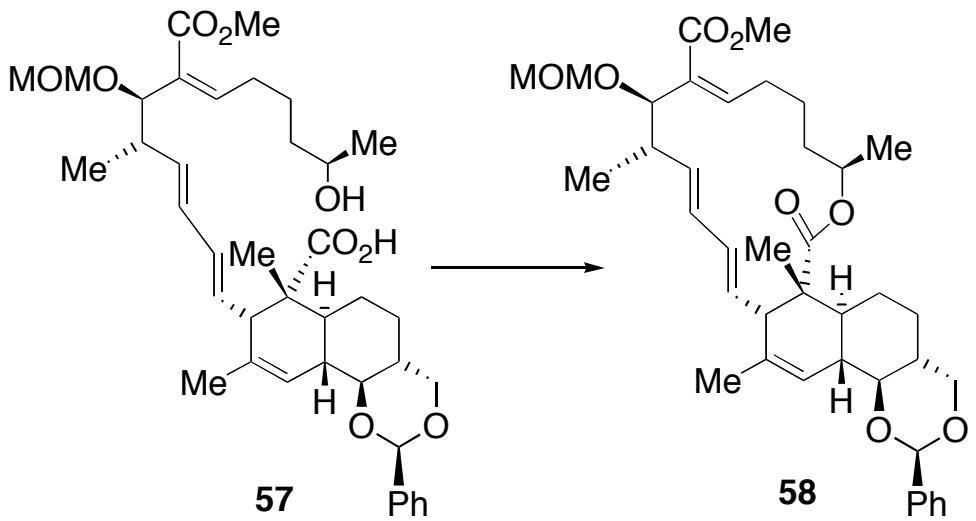

A solution of $57(59.4 \mathrm{mg}, 89.1 \mu \mathrm{mol})$ in $\mathrm{MeCN}(30 \mathrm{~mL})$ was added to a refluxing solution of 2chloro-1-methylpyridinium iodide $(114 \mathrm{mg}, 0.445 \mathrm{mmol})$ and $\mathrm{Et}_{3} \mathrm{~N}(0.12 \mathrm{~mL}, 0.89 \mathrm{mmol})$ in $\mathrm{MeCN}(12$ $\mathrm{mL}$ ) over $3.5 \mathrm{~h}$ by an addition funnel. The addition funnel was rinsed with $3 \mathrm{~mL}$ of $\mathrm{MeCN}$. The mixture was refluxed for an additional $3.5 \mathrm{~h}$. After being cooled to room temperature, the solution was concentrated in vacuo. The residue was purified by column chromatography on silica gel (EtOAc/hexane, 1:5) to give $46.6 \mathrm{mg}(81 \%)$ of $\mathbf{5 8}$ as a colorless oil: TLC, $\mathrm{R}_{f} 0.72$ (EtOAc/hexane, 2:1); $[\alpha]^{28}+44.9\left(c 0.96, \mathrm{CHCl}_{3}\right) ;{ }^{1} \mathrm{H}$ NMR $(300 \mathrm{MHz}) \delta 1.05-1.15(\mathrm{~m}, 3 \mathrm{H}), 1.14(\mathrm{~d}, J=7.1 \mathrm{~Hz}, 3 \mathrm{H}), 1.15(\mathrm{~s}$, $3 \mathrm{H}), 1.21(\mathrm{~d}, J=6.3 \mathrm{~Hz}, 3 \mathrm{H}), 1.35-1.52(\mathrm{~m}, 2 \mathrm{H}), 1.58-1.65(\mathrm{~m}, 2 \mathrm{H}), 1.62(\mathrm{~s}, 3 \mathrm{H}), 1.78-1.92(\mathrm{~m}, 2 \mathrm{H})$, 1.96-2.10 (m, 2H), 2.29-2.42 (m, 1H), $2.37(\mathrm{~d}, J=10.0 \mathrm{~Hz}, 1 \mathrm{H}), 2.55(\mathrm{ddd}, J=16.9,8.9,4.6 \mathrm{~Hz}, 1 \mathrm{H})$, $2.91(\mathrm{~m}, 1 \mathrm{H}), 3.31(\mathrm{dd}, J=9.9,9.9 \mathrm{~Hz}, 1 \mathrm{H}), 3.37(\mathrm{~s}, 3 \mathrm{H}), 3.63(\mathrm{dd}, J=11.0,11.0 \mathrm{~Hz}, 1 \mathrm{H}), 3.76(\mathrm{~s}, 3 \mathrm{H})$, $3.84(\mathrm{~d}, J=8.5 \mathrm{~Hz}, 1 \mathrm{H}), 4.12(\mathrm{dd}, J=11.0,4.1 \mathrm{~Hz}, 1 \mathrm{H}), 4.50,4.67(2 \mathrm{~d}, J=6.8 \mathrm{~Hz}, 1 \mathrm{H} \times 2), 4.65-4.77$ $(\mathrm{m}, 1 \mathrm{H}), 5.35(\mathrm{dd}, J=13.8,10.0 \mathrm{~Hz}, 1 \mathrm{H}), 5.57(\mathrm{~s}, 1 \mathrm{H}), 5.67-5.80(\mathrm{~m}, 2 \mathrm{H}), 5.72(\mathrm{~s}, 1 \mathrm{H}), 5.90-6.01(\mathrm{~m}$, 2H), 7.32-7.41 (m, 3H), 7.52-7.54 (m, 2H); ${ }^{13} \mathrm{C} \mathrm{NMR} \mathrm{(68} \mathrm{MHz)} \mathrm{\delta} \mathrm{15.6,} \mathrm{16.7,} \mathrm{19.2,} \mathrm{22.4,} \mathrm{25.1,} \mathrm{25.7,} \mathrm{26.2,}$ $28.6,34.8,38.2,38.4,41.6,42.5,48.4,51.3,54.4,55.6,71.1,72.0,84.6,84.6,94.0,101.5,119.4,126.0$ $\times 2,128.2 \times 2,128.3,128.6,131.0,131.1,133.1,133.5,135.6,138.7,145.9,167.4,174.2$; IR (neat) 3020, 2930, 2880, 2850, 1730, 1715, $1455 \mathrm{~cm}^{-1}$; HRMS calcd for $\mathrm{C}_{39} \mathrm{H}_{52} \mathrm{O}_{8}\left(\mathrm{M}^{+}\right) \mathrm{m} / z$ 648.3662, found 648.3654. 


\section{6,13,16,20,24-pentamethyl-14-oxatricyclo[14.8.0.0 $\left.{ }^{17,22}\right]$ tetracosa-2,4,8,23-tetraene-15-one (59).}

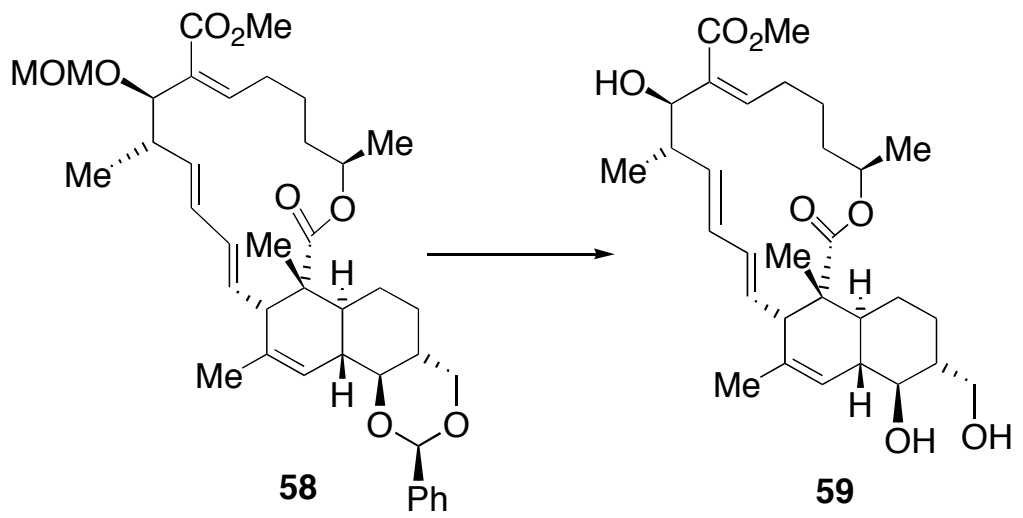

To a cooled $\left(0^{\circ} \mathrm{C}\right)$, stirred solution of $\mathbf{5 8}(15.1 \mathrm{mg}, 23.3 \mu \mathrm{mol})$ in THF $(0.6 \mathrm{~mL})$ was added $6 \mathrm{M}$ aqueous $\mathrm{HCl}(0.6 \mathrm{~mL})$. The mixture was stirred for $6 \mathrm{~h}$ and neutralized with saturated aqueous $\mathrm{NaHCO}_{3}$ $(10 \mathrm{~mL})$ at $0{ }^{\circ} \mathrm{C}$. This was extracted with $\mathrm{CH}_{2} \mathrm{Cl}_{2}(5 \mathrm{~mL} \times 3)$. The combined organic layers were dried and concentrated in vacuo. The residue was purified by column chromatography on silica gel (EtOAc/hexane, 1:2) to give $10.9 \mathrm{mg}(91 \%)$ of $\mathbf{5 9}$ as a colorless oil: TLC, $\mathbf{R}_{f} 0.32$ (EtOAc/hexane, 4:1); $[\alpha]_{\mathrm{D}}^{27.5}+86.3\left(c\right.$ 0.54, $\left.\mathrm{CHCl}_{3}\right) ;{ }^{1} \mathrm{H}$ NMR $(300 \mathrm{MHz}) \delta 0.99-1.19(\mathrm{~m}, 3 \mathrm{H}), 1.13(\mathrm{~s}, 3 \mathrm{H}), 1.20(\mathrm{~d}, J=6.9$ $\mathrm{Hz}, 3 \mathrm{H}), 1.22(\mathrm{~d}, J=6.3 \mathrm{~Hz}, 3 \mathrm{H}), 1.32-1.55(\mathrm{~m}, 2 \mathrm{H}), 1.57-1.90(\mathrm{~m}, 6 \mathrm{H}), 1.63(\mathrm{~s}, 3 \mathrm{H}), 2.36(\mathrm{~d}, J=10.0$ $\mathrm{Hz}, 1 \mathrm{H}), 2.44(\mathrm{ddd}, J=17.6,8.4,4.5 \mathrm{~Hz}, 1 \mathrm{H}), 2.57-2.73(\mathrm{~m}, 2 \mathrm{H}), 3.34(\mathrm{dd}, J=9.4,9.4 \mathrm{~Hz}, 1 \mathrm{H}), 3.63(\mathrm{~d}$, $J=8.5 \mathrm{~Hz}, 1 \mathrm{H}), 3.69(\mathrm{dd}, J=10.7,8.5 \mathrm{~Hz}, 1 \mathrm{H}), 3.77(\mathrm{dd}, J=10.7,3.4 \mathrm{~Hz}, 1 \mathrm{H}), 3.80(\mathrm{~s}, 3 \mathrm{H}), 4.72(\mathrm{~m}$, $1 \mathrm{H}), 5.32(\mathrm{dd}, J=14.1,10.0 \mathrm{~Hz}, 1 \mathrm{H}), 5.53(\mathrm{dd}, J=14.9,6.4 \mathrm{~Hz}, 1 \mathrm{H}), 5.71(\mathrm{~s}, 1 \mathrm{H}), 5.82-5.98(\mathrm{~m}, 3 \mathrm{H})$; ${ }^{13} \mathrm{C}$ NMR $(68 \mathrm{MHz}) \delta 15.6,16.6,19.2,22.6,25.3,26.2,26.8,28.5,35.1,37.5,40.4,45.4,46.4,48.4$, $51.4,54.4,68.8,71.0,79.3,83.4,119.7,127.8,131.2,132.3,133.2,133.5,135.8,147.1,167.7,174.3$; IR (neat) 3400, 3020, 2930, 2880, 1730, 1715, 1690, $1455 \mathrm{~cm}^{-1}$; HRMS calcd for $\mathrm{C}_{30} \mathrm{H}_{44} \mathrm{O}_{7}\left(\mathrm{M}^{+}\right) \mathrm{m} / z$ 516.3087, found 516.3077. 


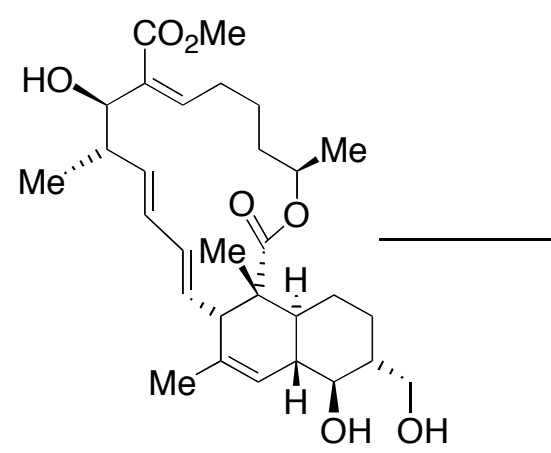

59

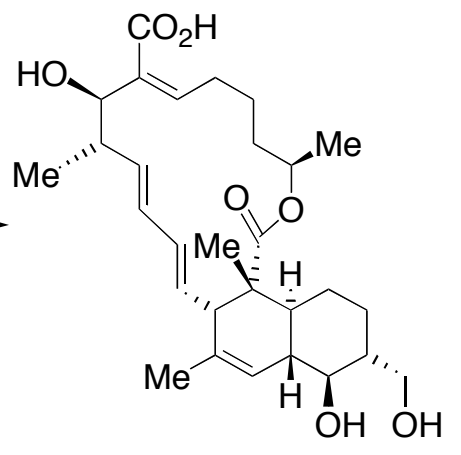

4

To a stirred solution of $\mathbf{5 9}(9.9 \mathrm{mg}, 19 \mu \mathrm{mol})$ in $\mathrm{MeOH}(1 \mathrm{~mL})$ was added $1 \mathrm{M}$ aqueous $\mathrm{NaOH}(0.5$ $\mathrm{mL}$ ). The mixture was heated to $50^{\circ} \mathrm{C}$ for $3 \mathrm{~h}$. After being cooled to room temperature, the mixture was acidified with $1 \mathrm{M}$ aqueous $\mathrm{HCl}$ to $\mathrm{pH} 2$ at $0{ }^{\circ} \mathrm{C}$. This was diluted with $1 \mathrm{M}$ aqueous $\mathrm{HCl}(10 \mathrm{~mL})$ and extracted with $\mathrm{CH}_{2} \mathrm{Cl}_{2}(5 \mathrm{~mL} \times 3)$. The combined organic layers were dried and concentrated in vacuo. The residue was purified by column chromatography on silica gel (acetone/hexane, 1:1 containing $1 \% \mathrm{AcOH})$ to give $9.1 \mathrm{mg}(94 \%)$ of 4 as a colorless solid: TLC, $\mathrm{R}_{f} 0.27\left(\mathrm{CHCl}_{3} / \mathrm{MeOH}, 10: 1\right.$ containing 1\% AcOH), 0.36 (acetone/hexane, 1:1 containing 1\% AcOH); $[\alpha]^{23.5}+94.9(c 0.23, \mathrm{MeOH})$; ${ }^{1} \mathrm{H}$ NMR $\left(270 \mathrm{MHz}\right.$, acetone- $\left.d_{6}\right) \delta 1.01-1.76(\mathrm{~m}, 10 \mathrm{H}), 1.13(\mathrm{~s}, 3 \mathrm{H}), 1.15(\mathrm{~d}, J=6.0 \mathrm{~Hz}, 3 \mathrm{H}), 1.23(\mathrm{~d}, J$ $=6.3 \mathrm{~Hz}, 3 \mathrm{H}), 1.61(\mathrm{~s}, 3 \mathrm{H}), 1.84(\mathrm{~m}, 1 \mathrm{H}), 2.40(\mathrm{~d}, J=10.0 \mathrm{~Hz}, 1 \mathrm{H}), 2.47-2.57(\mathrm{~m}, 2 \mathrm{H}), 2.60-2.73(\mathrm{~m}$, $1 \mathrm{H}), 3.22(\mathrm{dd}, J=9.3,9.3 \mathrm{~Hz}, 1 \mathrm{H}), 3.65(\mathrm{dd}, J=10.5,6.6 \mathrm{~Hz}, 1 \mathrm{H}), 3.72(\mathrm{dd}, J=10.5,5.1 \mathrm{~Hz}, 1 \mathrm{H}), 3.94$ $(\mathrm{d}, J=8.4 \mathrm{~Hz}, 1 \mathrm{H}), 4.71(\mathrm{~m}, 1 \mathrm{H}), 5.29(\mathrm{dd}, J=13.9,10.0 \mathrm{~Hz}, 1 \mathrm{H}), 5.70(\mathrm{dd}, J=14.6,6.2 \mathrm{~Hz}, 1 \mathrm{H}), 5.83$ $(\mathrm{s}, 1 \mathrm{H}), 5.83-6.00(\mathrm{~m}, 3 \mathrm{H}) ;{ }^{13} \mathrm{C} \mathrm{NMR}\left(75 \mathrm{MHz}\right.$, acetone- $\left.d_{6}\right) \delta 16.1,17.2,19.4,22.9,26.0,27.2,28.1$, $35.8,38.5,41.9,46.5,48.1,49.1,55.1,67.1,71.4,77.4,81.3,122.3,128.9,131.8,133.0,134.2,134.9$ 136.9, 144.4, 168.6, 174.4 (solvent peak overlapped one carbon peak, so one carbon was not detected); IR (KBr) 3420, 2930, 2860, 1720, 1700, $1460 \mathrm{~cm}^{-1}$; HRMS calcd for $\mathrm{C}_{29} \mathrm{H}_{42} \mathrm{O}_{7}\left(\mathrm{M}^{+}\right) \mathrm{m} / z$ 502.2930, found 502.2930 . 


\section{References}

(1) (a) Motozaki, T.; Sawamura, K.; Suzuki, A.; Yoshida, K.; Ueki, T.; Ohara, A.; Munakata, R.; Takao, K.; Tadano, K. Org. Lett. 2005, 7, 2265-2267. (b) Motozaki, T.; Sawamura, K.; Suzuki, A.; Yoshida, K.; Ueki, T.; Ohara, A.; Munakata, R.; Takao, K.; Tadano, K. Org. Lett. 2005, 7, 2261-2264. 